\title{
Trust-based Communication in Wireless Cooperative Relaying Networks
}

\author{
by \\ Reyhaneh Changiz, B.Sc. \\ A thesis submitted to the \\ Faculty of Graduate and Postdoctoral Affairs \\ in partial fulfillment of the requirements for the degree of
}

Master of Applied Science in Electrical and Computer Engineering

Ottawa-Carleton Institute for Electrical and Computer Engineering (OCIECE)

Department of Systems and Computer Engineering

Carleton University

Ottawa, Ontario,Canada, K1S 5B6

January, 2011

(C)Copyright 2011, Reyhaneh Changiz 
Library and Archives

Canada

Published Heritage Branch

395 Wellington Street Ottawa ON K1A ON4 Canada
Bibliothèque et

Archives Canada

Direction du

Patrimoine de l'édition

395, rue Wellington

Ottawa ON K1A ON4

Canada
Your file Votre reférence

ISBN: 978-0-494-79590-3

Our file Notre référence

ISBN: 978-0-494-79590-3
NOTICE:

The author has granted a nonexclusive license allowing Library and Archives Canada to reproduce, publish, archive, preserve, conserve, communicate to the public by telecommunication or on the Internet, loan, distribute and sell theses worldwide, for commercial or noncommercial purposes, in microform, paper, electronic and/or any other formats.

The author retains copyright ownership and moral rights in this thesis. Neither the thesis nor substantial extracts from it may be printed or otherwise reproduced without the author's permission.
AVIS:

L'auteur a accordé une licence non exclusive permettant à la Bibliothèque et Archives Canada de reproduire, publier, archiver, sauvegarder, conserver, transmettre au public par télécommunication ou par l'Internet, prêter, distribuer et vendre des thèses partout dans le monde, à des fins commerciales ou autres, sur support microforme, papier, électronique et/ou autres formats.

L'auteur conserve la propriété du droit d'auteur et des droits moraux qui protège cette thèse. $\mathrm{Ni}$ la thèse ni des extraits substantiels de celle-ci ne doivent être imprimés ou autrement reproduits sans son autorisation.
In compliance with the Canadian Privacy Act some supporting forms may have been removed from this thesis.

While these forms may be included in the document page count, their removal does not represent any loss of content from the thesis.
Conformément à la loi canadienne sur la protection de la vie privée, quelques formulaires secondaires ont été enlevés de cette thèse.

Bien que ces formulaires aient inclus dans la pagination, il n'y aura aucun contenu manquant. 


\section{Abstract}

Cooperative communication is a promising technique to improve the efficiency of wireless communication networks. To mitigate the security issues raised by cooperative communication, trust management is essential to monitor the behaviour of the entities. In this thesis, the focus is on trust establishment for relay nodes and, applying the trust values in the cross-layer design of cooperative relaying networks. Insufficiencies of conventional Bayesian methodology for cooperative communication is shown. Then, it is modified by incorporating the relay selection policy and wireless channel conditions among the network nodes. Furthermore, the relay selection in cooperative relaying networks will be the focus where a throughput optimal trust-based relay selection policy is devised which stabilizes the system for all the arrival rate vectors in the stability region. In the thesis it is proved that the proposed policy is optimal through analysis. Finally, the comparison of its performance with four other sub-optimal policies is provided. 
To my husband and beloved parents 


\section{Acknowledgments}

First of all, I would like to sincerely thank my supervisors, Professor Ioannis Lambadaris and Professor F. Richard Yu for their tremendous time and efforts spent in leading, supporting and encouraging me in the course of my thesis and study. It would not be possible for me to finish this work without their constant help and invaluable guidance throughout my study. I will always look up to them as an inspiration to my professional career.

I would express my gratitude to Dr. Peter Mason and Dr. Helen Tang from Defense R\&D Canada for their support to this work and valuable suggestions. Many thanks to my colleagues for their understanding and friendship during the time we spent together. Finally, I would like to express my special thanks to my husband, my parents and my brother for their continuous understanding, supporting and encouraging in my life.

Thank you all!! 


\section{Table of Contents}

$\begin{array}{lll}\text { Abstract } & \text { iii }\end{array}$

$\begin{array}{ll}\text { Acknowledgments } & \mathbf{v}\end{array}$

Table of Contents $\quad$ vi

List of Tables $\quad$ viii

$\begin{array}{ll}\text { List of Figures } & \text { ix }\end{array}$

List of Abbreviations $\quad \mathbf{x}$

List of Symbols $\quad x i$

1 Introduction $\quad 1$

1.1 Research Overview . . . . . . . . . . . . . . . . . . 1

1.2 Research Motivation and Research Objectives . . . . . . . . . . 4

1.3 Thesis Contributions . . . . . . . . . . . . . . . 6

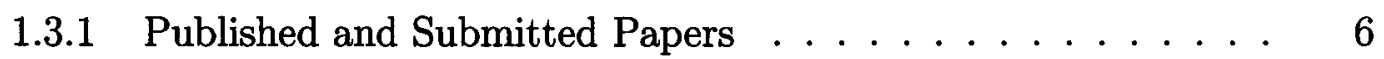

1.4 Thesis Organization . . . . . . . . . . . . . . . . . 7

2 Background and Related Works 9

2.1 Cooperative Communication . . . . . . . . . . . . . . . 9

2.1.1 Mobile Ad Hoc Networks (MANETs) . . . . . . . . . . 14

2.1.2 Relay Selection In Wireless Cooperative Relaying Networks . . 15

2.2 Security Challenges in Cooperative Communication . . . . . . . . 16

2.3 The Concept of Trust and Trust Management . . . . . . . . . . 20

2.3.1 Bayesian Methodology . . . . . . . . . . . . . 23

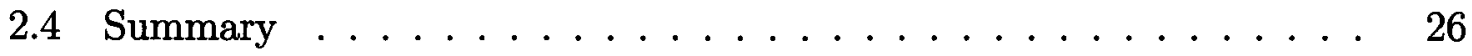


3 Trust Establishment in Cooperative Wireless Networks $\quad 27$

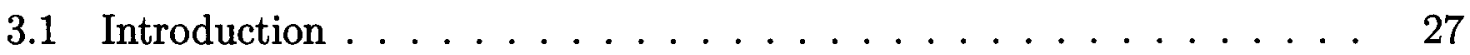

3.2 Model Description . . . . . . . . . . . . . . . . . 28

3.3 Trust Establishment For Wireless Cooperative Relaying Networks . . 32

3.3.1 Proposed Trust Establishment for Wireless Cooperative Relaying Networks . . . . . . . . . . . . . 34

3.4 Simulation Results and Discussions . . . . . . . . . . . . . 38

3.4.1 Case 1: Cooperative Relaying without Combining . . . . . . 38

3.4.2 Case 2: Cooperative Relaying with Combining . . . . . . . . 43

3.5 Summary . . . . . . . . . . . . . . . . . 48

4 Optimal Secure Relay Selection in Multiuser Cooperative Relaying Networks $\quad 49$

4.1 Introduction . . . . . . . . . . . . . . . . . . 49

4.1 .1 Problem Formulation . . . . . . . . . . . . . . . 51

4.2 Throughput-Optimal Secure Relay Selection Policy . . . . . . . . . 60

4.2 .1 Optimal Policy . . . . . . . . . . . . . . . . . 60

4.2.2 Bipartite Graph Representation of Optimal Policy . . . . . . . 63

4.3 Simulation Results . . . . . . . . . . . . . . . . . 65

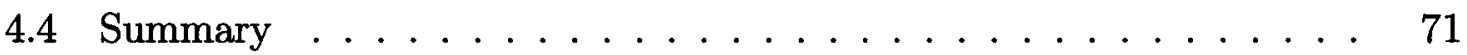

5 Conclusions and Future works $\quad 73$

$\begin{array}{ll}\text { List of References } & \mathbf{7 7}\end{array}$

$\begin{array}{lr}\text { Appendix A Simulation Programs } & 84\end{array}$ 


\section{List of Tables}

3.1 Relay trusts in system without combining (case 1) . . . . . . . 40

3.2 Relay trusts in system with combining (case 2) . . . . . . . . 44 


\section{List of Figures}

1.1 Cooperative communication $[1] \ldots \ldots \ldots \ldots$

3.1 The cooperative communication model. . . . . . . . . . . . . 30

3.2 Conventional Bayesian trust establishment in a simple cooperative sys-

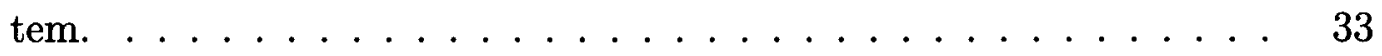

3.3 Trust establishment in the low SNR regime in case $1 . \ldots . . . . .39$

3.4 Trust establishment in the high SNR regime in case 1. . . . . . . . 40

3.5 Trust tracking with time in case $1 . \ldots \ldots \ldots 4 \ldots \ldots$

3.6 Trust tracking with and without using a sliding window in case 1. . . 42

3.7 Trust establishment in high SNR regime in case 2. . . . . . . . . . 43

3.8 Trust establishment in low SNR regime in case 2. . . . . . . . . 44

3.9 Trust tracking with time in system in case $2 \ldots \ldots \ldots \ldots$

3.10 Trust tracking with and without using a sliding window in case 2. . . 46

3.11 Relays trust of multi-user system in high SNR regimes . . . . . . 47

3.12 Relays trust of multi-user system in high SNR regimes . . . . . . 48

4.1 The model of cooperative communication used in this chapter . . . . 52

4.2 The model of cooperative communication used in the example . . . 59

4.3 Comparison of stability region for trusted and untrusted relaying . . . 59

4.4 The bipartite graph representation of problem $\mathbf{P} \ldots \ldots \ldots$. . . . . 64

4.5 The grid cell structure . . . . . . . . . . . . . . 67

4.6 Average queue occupancy versus arrival rate in case $1 \ldots \ldots 9$

4.7 Average queue occupancy versus arrival rate in case $2 \ldots \ldots 69$

4.8 Average queue occupancy versus arrival rate in case $3 \ldots \ldots 70$

4.9 Average queue occupancy versus arrival rate in case $4 \ldots \ldots$ 


\section{List of Abbreviations}

$\begin{array}{ll}\text { MIMO } & \text { Multiple-Input Multiple-Output } \\ \text { MRC } & \text { Maximum Ratio Combining } \\ \text { DF } & \text { Decode-and-Forward } \\ \text { AF } & \text { Amplify-and-Forward } \\ \text { SNR } & \text { Signal-to-Noise Ratio } \\ \text { STC } & \text { Space Time Coding } \\ \text { BPSK } & \text { Binary Phase Shift Keying } \\ \text { BER } & \text { Bit Error Rate } \\ \text { CSI } & \text { Channel State Information } \\ \text { AWGN } & \text { Additive White Gaussian Noise } \\ \text { dB } & \text { Decibel } \\ \text { MANET } & \text { Mobile Ad hoc Network } \\ \text { ACK } & \text { Positive Acknowledgement } \\ \text { NACK } & \text { Negative Acknowledgement } \\ \text { P2P } & \text { Peer-to-Peer } \\ \text { QoS } & \text { Quality of Service } \\ \text { PSD } & \text { Power Spectral Density } \\ \text { IP } & \text { Integer Programming } \\ \text { STO } & \text { Secure Throughput Optimal } \\ \text { NSTO } & \text { Non-Secure Throughput Optimal } \\ \text { SITO } & \text { Secure Instantaneous Throughput Optimal } \\ \text { STO-PTR } & \text { Throughput Optimal with Pure Trusted Relays } \\ \text { NC } & \text { Non-Cooperative } \\ \text { NP } & \text { Non-Polynomial } \\ \text { NS } & \text { Non-Secure }\end{array}$




\section{List of Symbols}

$\begin{array}{ll}S & \text { Source } \\ D & \text { Destination } \\ r & \text { Relay } \\ N & \text { Number of Users } \\ R & \text { Number of Relays } \\ r^{*} & \text { Best Relay } \\ Q_{\imath} & \text { Random Variable of Maliciousness of Relay } i \\ q_{\imath} & \text { Maliciousness probability of Relay } i \\ S-D & \text { Source to Destination link } \\ \frac{a}{2} & \text { Path Loss Exponent } \\ Z_{D} & \text { Additive Zero Mean Gaussian Noise at Node } D \\ \ell(t) & \text { Number of Packets Successfully Transmitted by a Node at Iteration } t \\ \ell_{\imath}(t) & \text { Number of Packets Successfully Transmitted by Relay } i \text { at Iteration } t \\ k(t) & \text { Number of Packets Received by a Node } \\ k_{\imath}(t) & \text { Number of Observations Regarding Relay } i \text { During Iteration } t \\ f_{t}(\bar{q}) & \text { Estimated Distribution Function of Trust at Iteration } t \\ T_{\imath}(t) & \text { Trust of Relay } i \text { at Iteration } T \\ X & \text { Transmitted Signal } \\ Y & \text { Received Signal } \\ d_{S-D} & \text { Distance between Source and Destination } \\ d_{S-r_{\imath}} & \text { Distance between Source and Relay } i \\ d_{r_{2}-D} & \text { Distance between Relay } i \text { and Destination } \\ H_{S-r} & \text { Channel Gain of the Link from Node } S \text { to } r \\ h_{S-r} & \text { Fading Coefficient of the link between Node } S \text { to } r \\ P_{\imath} & \text { Transmit Power of node } i \\ \gamma_{t h r} & \text { Threshold SNR for Successful Transmission } \\ \gamma_{S-D} & \text { Average SNR of Received Signal of the Link } S-D \\ \gamma_{S-r_{\imath}} & \text { Average SNR of Received Signal of the Link Source to Relay } i \\ \gamma_{r_{\imath}-D} & \text { Average SNR of Received Signal of the Link Relay } i \text { to } D \\ & \end{array}$




$\begin{array}{ll}T_{n, 2} & \text { Trust of Relay } i \text { with Respect to User } n \\ W & \text { Noise Power } \\ \Omega & \text { Set of Relay Nodes Who Have Decoded The Packet Correctly } \\ \Theta_{2}^{J} & \text { Event Representing the Selection of Relay } i \text { in case } j \\ \ell_{\Theta_{i}}^{J}(t) & \text { Number of Times that Relay } i \text { is Selected in Iteration } t \\ \ell_{\gamma_{i}}^{J}(t) & \text { Number of Times that SNR of Link } r_{\imath}-D \text { is Greater than } \gamma_{t h r} \\ \omega & \text { Window Size } \\ W_{n} & \text { Transmission Bandwidth of User } n \text { Channel } \\ N_{0} / 2 & \text { Noise Power Spectral Density } \\ C_{n} & \text { Channel Assigned to User } n \\ A_{n}(t) & \text { Arrival Process to User } n \text { at Time Slot } t \\ \lambda_{n} & \text { Mean of the Arrival Process to User } n \\ U_{n}(t) & \text { Queue Length Process of User } n \\ R_{n, D}(t) & \text { Achievable Bit Rate at Link } \ell_{n, D} \\ w_{n, D}(t) & \text { Number of Successful Packet Transmissions at Time Slot } t \text { from user } n \text { to } D \\ I_{n, D}(t) & \text { Indicator Variable when User } n \text { transmits directly to } D \text { at time } t \\ I_{n, r}(t) & \text { Indicator Variable when } n \text { Selects Relay node } r \text { at Time } t \text { for Cooperation } \\ \mu(t) & \text { service process } \\ \pi & \text { Relay Selection Policy } \\ \Lambda_{\pi} & \text { Stability Region for Policy } \pi \\ \Lambda & \text { Network Stability Region } \\ \pi^{*} & \text { Throughput Optimal Policy } \\ \Lambda_{S e c u r e} & \text { Stability Region of a Secure Relaying System } \\ \Lambda_{N o n-S e c u r e} & \text { Stability Region of a Non-Secure Relaying System } \\ \mathbf{P} & \text { Optimization Problem } \\ \Theta & \text { Policy in which } I_{n, D}(t) \text { and } I_{n, r}(t) \text { are the Solution to Problem } \mathbf{P} \\ \Delta(t) & \text { Lyapunov Drift } \\ V & \text { Vertices of a Graph } \\ E & \text { Set of Edges } \\ e & \text { Edge Weight }\end{array}$




\section{Chapter 1}

\section{Introduction}

\subsection{Research Overview}

Wireless communication has captured a lot of attention over the last few decades from both academia and industry. Today, a large body of the research done in the field of communication engineering is dedicated to wireless communications. We can see a huge advance over a few years, in the theory, methods, algorithms and protocols proposed for different type of wireless networks such as cellular networks, Ad Hoc networks, sensor networks, etc. The main result of such effort is to develop new wireless technologies, new services, new applications and new devices which is of great importance in communication industry.

The purpose of most of the research in wireless communication is to devise new approaches to maximize the efficiency of the system from different perspectives. In other words, the main goal is to get the maximum benefits (e.g., throughput) out of the system with a limited amount of resources (e.g., bandwidth and power). Cooperative communication is an approach to this end.

Wireless communication networks are traditionally conceptualized as consisting of a series of point to point communications between communicating entities. For example, in wireless cellular networks the communicating entities are the set of users and a base station where users individually communicate with the base stations and vice versa. Another example is Ad Hoc networks in which communication of two wireless entities is composed of routing of data packets from the source node to the destination node over some point to point communication links. Cooperative communication architectures deviate from this approach. In wireless cooperative communication, we are concerned with a wireless network in which users may improve their effective quality 
of service (e.g., achievable rate, bit error rates or outage probability) via cooperation.

In general, in cooperative communication it is assumed that each user transmits its own data as well as relaying the other user's data. In other words, an entity which is playing the role of a relay acts cooperatively to help other entities to transmit their data to the destination. Figure 1.1 [1] represents a basic cooperative system.

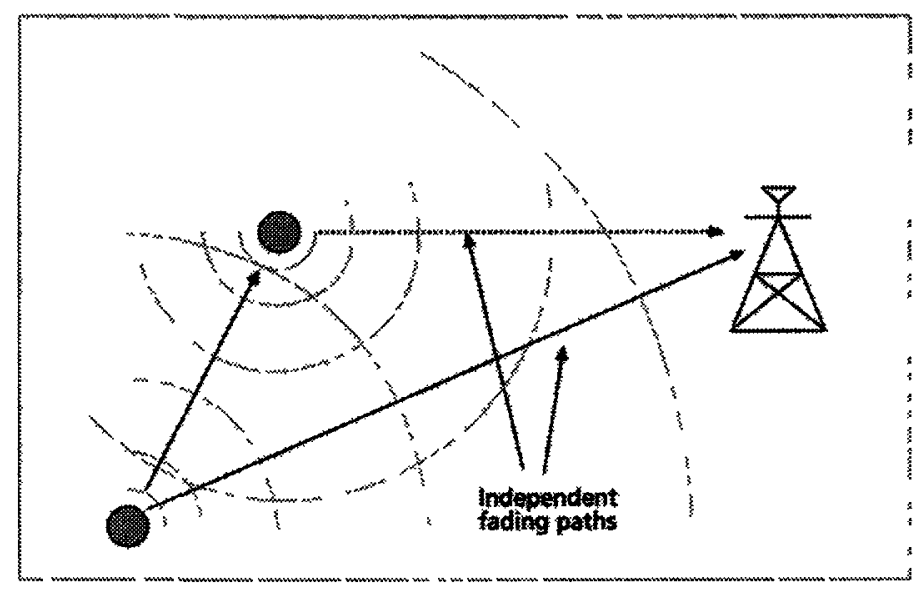

Figure 1.1: Cooperative communication [1]

The main attribute of the wireless channel that helps in cooperative communication is the broadcast nature of it. In other words, the broadcasted signal from the the source node is heard by both the destination and the relay node. Then, the relay node does some process on the received signal and then broadcasts it to the destination node. The destination node uses the received signal from the source and the relay node to detect the transmitted symbols from the source. In fact, the cooperative communication uses the relays as virtual antennas to forward the information of other nodes. That is why cooperative communication is also known as virtual multiple-input multiple-output (MIMO) in wireless communication literature [1]. The advantages of MIMO systems in improving the efficiency and reliability of wireless communication systems have been widely acknowledged in literature and was incorporated in wireless communication standards e.g., Alamouti signaling. However, because of size, cost and hardware implementation limitations, it is not feasible to support MIMO on wireless handsets or wireless sensor nodes. That is the point where virtual MIMO or cooperative relaying comes into play where relays act as virtual antennas of the source node. Thus, many advantages of wireless MIMO systems like spatial diversity is also achievable in cooperative relaying in which case it is called cooperative diversity. In 
summary, cooperative communication involves the following two main concepts [2]:

- Using relay nodes to provide spatial diversity in a fading environment.

- Devising a collaborative scheme where nodes help each other to communicate by acting as relays.

Note that cooperative relaying not only improves the system from reliability and efficiency point of view, it can lead to coverage increase of wireless access networks specially in cellular networks. Cooperative communication is now incorporated in the IEEE $802.16 \mathrm{j}$ standard to increase system coverage and performance through the use of relay stations located between the users and base station [3].

Although cooperative communication can provide significant performance and coverage improvements, it may raise some security issues due to the nature of the system in which the source node has to trust some relay nodes for cooperation. The security vulnerabilities can be addressed either by prevention-based or detection-based techniques. In prevention-based techniques, the goal is to employ some prevention techniques such as authentication, particularly data origin authentication, and integrity protection. In fact, these types of tools are the security mechanisms that act at the front line of defence against security attacks in cooperative communication networks. Detection-based techniques which are the main focus of this thesis are used to support the provision-based techniques. In fact, detection-based techniques are applied to detect the misbehaving relay nodes in cases where provision-based techniques fail. Intrusion or anomaly detection tools are applied at this stage of defence in communication networks. In summary, detection-based techniques try to identify the anomalous behaviour of malicious relay nodes and then react properly to those relay nodes. Trust establishment or trust evaluation techniques are one of the main tools which are used as a detection-based technique in wireless networks. In fact, trust establishment is a mechanism that can distinguish the misbehaving relay nodes from the benign ones. In this mechanism, each relay node is associated with a real value from the interval $[0,1]$ called trust, representing to what extent it is trustworthy in cooperation. In general, trust is defined as the probability that the trustee performs a specific action that trustor expects [4]. In cooperative wireless systems, each relay node is a trustee which is expected to forward the received packets to the destination when it is selected for cooperation. Trust management is a scheme to evaluate and assign a trust value to every entity in the network which enables the trustor (e.g., the 
source) to detect misbehaving nodes. Lots of trust establishment methods (as well as ours) are based on Bayesian framework which is discussed in Chapter 2 in more detail.

This thesis is consisting of two major sections: In the first section of the thesis discussed in Chapter 3, a Bayesian trust management method for wireless cooperative networks is proposed taking into account both the channel state information as well as the employed relay selection policy. It is shown that conventional Bayesian methodology is insufficient for the cooperative communication paradigm, as it is biased by the channel conditions and relay selection decision processes. Therefore, we modify the conventional Bayesian trust management method by incorporating not only the relay selection policy but also the dynamic variations of wireless channel conditions among the source, relays and destination. Simulation results are presented to show the effectiveness of the proposed scheme.

In the second part of the thesis which is discussed in Chapter 4, we try to employ the trust associated to each relay node to devise an optimal secure relay selection policy in multiuser cooperative wireless networks. To this end, a general discrete time model for such networks is introduced which takes into account the dynamic variations of the channel state, the stochastic malicious behaviour of relay nodes as well as stochastic arrival of data packets into the system. We first, demonstrate that malicious behaviour of the relay nodes causes the stable throughput region of the system to shrink. Then, we will propose a throughput optimal dynamic relay selection policy and show that the optimal policy is equivalent to finding the maximum weighted matching in a weighted bipartite graph at each time slot. We also use simulations to compare the performance of the proposed policy with four other suboptimal policies in terms of average queue occupancy (or equivalently, queueing delay) and stable throughput region.

\subsection{Research Motivation and Research Objectives}

The following problems noticed in cooperative communication systems motivated us to carry out this research:

- Malicious nodes behaviour in a cooperative wireless network may deteriorate the security in cooperative communication and cause the cooperation to cease. 
In fact, malicious relay nodes act as a bump in the network and cause intentional delay in the system.

- Malicious relay nodes are tend to hide their malicious behaviour behind the inherent variability and uncertainty of wireless channel. Thus, detecting the malicious relay nodes is not a straightforward task.

- While there exists a rich body of research on the area of trust establishment in wireless Ad Hoc networks, there is not much work in wireless cooperative networks.

- The trust establishment method should consider the available information about the channel states as well as relay selection decisions to determine an unbiased trust value for relay nodes.

- In most of the relay selection policies considered in literature, the relay nodes are mostly considered as pure trusted relays who obey the network controller decisions all the time. This is not the case in a compromised network.

- Although there exists some proposed routing algorithms for Ad Hoc networks based on the trust values [5], to best of our knowledge, there is no work in cooperative networks which incorporates the trust values in resource allocation (e.g. relay assignment) decisions.

In order to address the above problems, we propose an efficient and easy to implement trust establishment scheme in wireless cooperative networks. As mentioned earlier, the proposed method is based on Bayesian framework and it is applicable to cooperative systems with any relay selection policy whose decisions are just based on the available information at the current cooperative time frame. We also propose a relay assignment policy which incorporates the trust values associated to each relay node. The proposed relay assignment policy is inspired by the well known backpressure algorithm [6]. For the proposed policy we will first prove its optimality in terms of stable throughput region and then evaluate its performance with some other sub-optimal policies in terms of throughput region and average queueing delay. 


\subsection{Thesis Contributions}

As we mentioned before, the thesis contributions are into two directions: Based on the above objectives, we advance a detection-based security technique in cooperative wireless networks to derive the trust values associated to the relay nodes. The proposed scheme is based on the Bayesian trust establishment framework. We first, introduce the proposed method for a cooperative relaying system in which the quality of the direct link between source and destination is very poor. Then, we will extend the method for a system in which the destination performs combining on the received signals from the source and the relay node. We then examine the proposed method for a system with multiple users as well as multiple relays and by using simulations we demonstrate the effectiveness of the proposed scheme.

We also devised an optimal relay selection policy in a relaying system with malicious relay nodes. We first show how maliciousness of relay nodes can result in shrinking of the stable throughput region. Then, we propose a throughput optimal relay selection policy which considers queue lengths, channel state and trust values associated to the relay nodes. In the model considered in the relay selection problem, the term trust is a general term which represents the reliability of the relay nodes for cooperation. Those values may be drawn by using any trust establishment method. We proved that the proposed policy is throughput optimal and the optimal policy is equivalent to determination of a maximum matching in a weighted bipartite graph at each time slot. We also used simulations to compare the performance of the proposed policy with that of four other sub-optimal relay selection policies in terms of average queue backlog and stability region.

\subsubsection{Published and Submitted Papers}

Based on this work, the following papers have been published or submitted:

- R. Changiz, H. Halabian, F.R. Yu, I. Lambadaris and H. Tang, "Trust Management in Wireless Mobile Networks with Cooperative Communications," in Proc. Sixth IEEE/IFIP International Symposıum on Trusted Computıng and Communications (TrustCom'10), HongKong, P.R. China, Dec. 2010.

- R. Changiz, H. Halabian, F.R. Yu, I. Lambadaris, H. Tang and P.C. Mason, 
"Trust Establishment in Cooperative Wireless Networks," in Proc. IEEE Milztary Communcations Conference (Milcom'10), San Jose, CA, USA, Nov. 2010.

- R. Changiz, H. Halabian, F.R. Yu, I. Lambadaris and H. Tang "Trust Management in Wireless Cooperative Relay Networks," submitted to Wireless Communıcations and Mobıle Computıng, Jan. 2010.

- R. Changiz, H. Halabian, F.R. Yu, I. Lambadaris and H. Tang "Optimal Secure Relay Selection in Multiuser Cooperative Relaying Networks," submitted to ACM/Springer Wireless Networks, Jan. 2010.

\subsection{Thesis Organization}

The rest of the thesis is organized as follows.

- Chapter 2: In this chapter titled "Background and Related Works", we describe the research background of this thesis. We introduce the concepts of cooperative communication and review the fundamental research works in the area of relay selection in cooperative relaying networks. We discuss about the security issues in cooperative communication, the concept of trust and trust establishment in wireless networks. We also review the concept of Bayesian trust establishment framework as the proposed trust establishment method in this thesis is based on Bayesian framework.

- Chapter 3: In this chapter titled "Trust Establishment in Wireless Cooperative Networks", we describe our proposed trust establishment scheme in detail by presenting the system model, the equations for the proposed method and finally the simulation results.

- Chapter 4: In this chapter, titled "Optimal Secure Relay Selection in Multiuser Cooperative Relaying Networks", we present a throughput optimal secure relay selection policy for multiuser cooperative relaying networks. We will provide the analysis and the proofs as well as simulation results where we compare the performance of the proposed policy with some other cooperative and noncooperative policies.

- Chapter 5: This chapter summarizes the conclusions of the thesis and highlights the next steps for future works. 
Our simulation codes are presented in Appendix A. 


\section{Chapter 2}

\section{Background and Related Works}

In this chapter, the background for this thesis is provided by an overview on cooperative communication in networks, mobile Ad Hoc networks, relay selection and how the behaviour of the relays can affect it and consequently affect the performance of the system. Also, security issues related to network employing cooperative communication and how it can severely affect the cooperative communication and degrade the performance of the system are brought in this section. We will discuss the schemes to deal with security issues, differentiating between the detection-based and preventionbased techniques and describe the importance of trust management in the system. Trust and trust management as two main concepts used in detection-based techniques are reviewed. In this regard, Bayesian framework, a well known methodology which will be used in the proposed trust establishment in this thesis is studied.

Also we will review the related work in the literature that has been performed and ideas developed in this area.

\subsection{Cooperative Communication}

Cooperative communication or cooperative diversity has become popular in communication networks in the recent years. In fact, cooperative communication is now considered as a promising technique to improve the performance of wireless communication systems in terms of throughput, efficiency, coverage and reliability. Not only it mitigates the effect of fading, but also fading is viewed positively to provide diversity gain.

In this type of communication, one or more relay nodes are involved to cooperatively transmit the source information to the destination. It has been shown that 
cooperation can lead to capacity improvements by providing spatial diversity $[2,7-9]$ and higher efficiency due to spatial multiplexing. The users may increase their quality of service (QoS) from the physical layer perspective which is measured bit error rate, block error rate, outage probability, etc., by means of cooperative communication. In this type of communication, each user will act as an assistant for other users and can transmit its own data as well. This makes use of the broadcast nature of the wireless medium, as the adjacent nodes may also hear and detect the signals transmitted by the source. In Figure 1.1, we can see a set of simple cooperative communication components consisting a source, a relay and a destination. In a cooperative network, one or more relays who have received the transmitted signal will forward the received signal to the destination. The destination will combine the signal received from the relay with the one received directly form the source and detects the transmitted signal. Using relays in cooperative communication eliminates the necessity of having multiple antennas at each node. The advantages of MIMO systems have been shown and are widely accepted. Two of the advantages using these systems are performance improvement of the system in terms of links reliability and increasing spectral efficiency. Using multiple antennas at either one or two side of the transmission link, i.e., transmitter and/or receiver have been incorporated in deploying cellular applications, significantly in base stations.

In contrast, in cooperative communication, spatial diversity can be gained without the need to deploy multiple antennas in a device. In fact, due to space limitation and power constraints, deploying multiple antennas in a mobile device is not always an easy task.

In cooperative communication, the single antenna node will share its own antenna among other users to mimic the MIMO systems and create a virtual environment similar to that. In this type of system, the user can act as both transmitter and a relay. Consequently, the spatial diversity is achieved when combining two signals which go through two independent fading paths. To combat the effect of wireless network constraints such as channel quality issue and resource limitations, having users share their resource and cooperate in other users data transmission is a positive approach. In [10], we can see that spatial diversity in the multi-user system will be correspondingly obtained in cooperative communication by permitting users with diverse fading paths, cooperate in transmitting each other's data to the destination. Although a user itself cannot create spatial diversity, exploiting one or more relays to forward the 
overheard version of the signal will cause the chance of transmission failure reduce [1]. Therefore, the goal of cooperative communication is to provide with multiple independently faded signal at the destination, so that it can perform combining on the received diverse versions of the signal (e.g. Maximum Ratio Combining [11]).

A cooperative system is similar to MIMO in terms of providing diversity gains but they are different in the following aspects. In the MIMO, there exist multiple antennas at either one or both of transmitter or receiver which is not the case in cooperative communication. In the cooperative communication, single antenna nodes perform the transmission to cooperate with each other. In this environment, the users do not transmit their data to the destination at the same time since they have to have another user retransmit the overheard data. An interesting issue which is of great importance in cooperative communication is relay selection. The relay selection may be complicated because the behaviour or performance of the relay may change over time. This topic will be discussed in more detail in section 2.1.2. In the cooperative communication, nodes can be multi-functional since they can serve as either relay, source or destination. They have the ability to forward other nodes information as well as transmitting their own information. As stated in [1], Cooperative communication leads to a trade-off in transmit power and code rates. Although the user has to transmit for both users at higher power when it is in cooperative mode compared to non-cooperative mode, the baseline transmit power of both users will decrease.

The main key idea behind cooperative communication is referred to the well known work of cover and EL Gamal which explores the information theoretic aspects of the relay channel [12]. Their capacity analysis was related to a channel consisting of three nodes, a source, a destination and a relay. In this type of communication the relays would act as pure relays and do not inject traffic into system. The concept of wireless fading channel was not considered in their model. As the authors discuss in [1], many works have a different emphasis on a desire not to consider the relays exclusively for the purpose of helping the main channel in their proposed protocols. Also, recent developments are driven by the concept of fading channel. However, since implementing those protocols employing users as relays is relatively complicated, the protocols deployed in realistic environments and standards such as IEEE $802.16 \mathrm{j}$ will use relays that only perform the task of relaying $[3,13]$. For the purpose of employing the users to relay other nodes information, the authors in [14] have proposed a user cooperation protocol to increase the uplink capacity and decrease the uplink 
outage probability at a given rate. Cooperative communication leads to noticeable performance gains. These improvement include but are not limited to increasing link reliability, spectral efficiency, capacity region, and transmission range [15]. In terms of cooperative diversity, the channel code rate will increase due to an improvement in spectral efficiency for each user despite the fact that the user has to transmit its own signal as well as its partners signal. Moreover, as can be implied from the previous discussions, the baseline transmit power reduction is observed on account of diversity gain, although more power might be required for each node as it transmits for another user as well. The authors in [1] show that cooperative communication results in substantial improvement in terms of error rate even while the channel quality of the two users is not better than the uplink channel. Cooperative communication is a cost effective solution to combat the effect of shadowing loss and enables users at the low coverage regions to overcome transmission problems and facilitates providing fair quality of service to all users [16]. Also, [8] shows that cooperative diversity protocols result in large power or energy savings.

Some of the main cooperative signalling methods are discussed in [1] which are going to be reviewed in the following. Data transmission between the source and destination via a relay can be performed through different modes as Decode and Forward, Amplify and Forward and Coded Cooperation. There are also, other hybrid schemes inspired by these main scheme [17]. In the schemes above, relays forward the received information to the destination. The destination not only does not consider the received signal from the relay (other path) as a noise or interference, but also it benefits from it in the way that it combines the received signal coming through the original direct link from source to destination. The signal combining substantially can take place in frequency, time or spatial domain [18].

The main cooperative communication modes characteristics can be summarized as follows.

- Decode and Forward: in this method, the relay decodes the received noisy signal transmitted by the source as an attempt to correctly detect the source information. It then, re-encodes it and retransmits the message to the destination. It can be considered the closest method to traditional relaying. Decode and Forward (DF), also known as Detect and Forward, may add some complexity but has a good performance in low Signal to Noise Ratio (SNR) regime. $[8,19,20]$ incorporate single relays to transmit the signal to the destination while [21-23] 
deal with multiple relays to perform forwarding the signal to the destination at the same time.

- Amplify and Forward: in this method, relay receives a noisy signal from the source. It attempts to amplify and retransmits the noisy received signal to the destination. This scheme is relatively simple but, it has performance degradation in low SNR regimes. That is, the noise is also amplified at the relay.

- Coded Cooperation: in this method, different portions of each users code word is sent through two independent fading paths. This will incorporate cooperation into channel coding. In fact, if possible, user transmits incremental redundancy for its partner, otherwise, the system operates in non-cooperative mode. The efficiency key of this method is that it works without any feedback between the users and the code design has made it possible.

Different cooperative diversity protocols have been developed. What makes each one distinct is the type of processing used at the intermediate nodes responsible for relaying and different methods of combining employed at the destination node. Main protocols include, fixed relaying, selection relaying, incremental relaying, and opportunistic relaying [24]. In fixed relaying, relay forwards the received signal to the destination regardless of the channel condition between the relay and the destination. Standard AF or DF communication schemes are examples of methods lying in this category [8]. In selection relaying, channel state from the relay to the destination plays a role. If the channel quality is bad, the relay does not forward any signal to the destination. Incremental relaying exploits limited feedback from the destination node, e.g., a bit as an indicator of the success or failure of the direct transmission. If the destination provides a negative acknowledgment via feedback, the relay retransmits to exploit spatial diversity by combining the signals received from the source and the relay at the destination [25]. Opportunistic relaying requires the selection of the best relay node among the several available relays. In a three-node relay channel, best relay is selected out of all the intermediate relay nodes to be used for data transmission instead of using all the relays.

Many security concepts used in this thesis has been inspired by the issues in Mobile Ad Hoc Networks (MANETs). Thus, a brief review on MANETs is brought in as follows. 


\subsubsection{Mobile Ad Hoc Networks (MANETs)}

A MANET is a network without any infrastructure which is formed temporarily by a set of wireless mobile nodes. They dynamically establish their own network without any central administration [26]. Each entity in MANET is free to move independently in any direction.Therefore, frequent topology changes happen in such networks. A node in MANET can act as a relay to transmit other nodes' data from one source to a destination. They can also be a source or destination of data which can inject data into the system. Based on the distributed nature and lack of centralized administrator or controller, nodes in the MANET have to cooperate with each other to achieve the main objective of such systems. Accordingly, cooperative communication or diversity arises naturally in Ad Hoc networks. Noticeable power saving and delay reduction for power savings will take place with inexpensive, simple and mobile nodes, while it supports decentralized routing and control algorithms [27,28]. MANET imposes constraints on the network architecture in terms of operational physical layer transmission channel originating from only one transmitter and the concurrent transmission from multiple transmitters since it can potentially result in interference, collision, and distortion at the destination which further leads to performance degradation. However, cooperative communication is not affected by these constraints, and thus, there is a great deal of room for design of network architectures in MANET that integrates cooperation [29]. Authors in [29,30] have proposed two MANET models using cooperative communication and showed the advantages of their proposed model in terms of flexibility compared to the standard MANETs. In one of the models, cooperative transmission is centrally activated and controlled by cluster access points and all nodes communicate through this access point, which deal with routing to other clusters using multiple gateway nodes and hence provide significant cooperative gains compared to the single gateway solution. Links with better performances results in a better network connectivity when it is compared to multi-hop solutions. In the second model, a random source conveys extra control information and link parameters in the message to enable recipients to self-select and form a random cooperative cluster in a MANET. The nodes in the cluster can rely on the synchronization data available in the source packet. 


\subsubsection{Relay Selection In Wireless Cooperative Relaying Net- works}

Other than information theoretic studies of cooperative wireless systems (such as the works in $[2,7,8,12,31,32])$, there is a large body of research in cooperative communication whose main goal is to devise efficient cooperative relaying protocols. In these types of works, the main problem is to find optimal resource (for example, power, channel, relay, etc.) allocation schemes according to an objective function as well as the constraints on the underlying model. Optimal relay selection is one of such problems which is of great importance in cooperative wireless networks.

In fact, selection of an appropriate relay node for cooperation affects the performance of the system significantly. Most of the works in this area focus on the physical layer issues and usually study the bit error rate, capacity and outage behaviour of the proposed schemes [33-41].

In [33], a concept of selection cooperation is presented and it is demonstrated that cooperative relay selection outperforms the distributed Space Time Coding (STC) scheme. Authors in [34] proposed a relay selection strategy which minimizes the outage probability under a short-term power constraint. The reference [35] studies diversity order of many single-relay selection schemes and then generalizes the idea of single relay selection to multiple relay selection. The work [36] studies the relay selection from the game theoretic point of view and proposes a distributed buyer/seller game which addresses two questions: First, which relay nodes should be included in cooperation; second, what is the optimal power level. In [42], a distributed relay selection scheme that requires limited network knowledge is proposed which is based on instantaneous SNRs. In [37], the proposed relay selection policy minimize the total transmission time of a fixed amount of data. Opportunistic relay selection scheme was studied in [38,43-45]. In [43], it is shown that opportunistic relay selection scheme achieves significantly better outage behaviour than the conventional allparticipate scheme. In [44] and [45], it is demonstrated that under an aggregate power constraint, opportunistic relay selection scheme is globally outage-optimal. Authors in [38] have shown that in a multiple-relay network, the opportunistic relay selection outperforms any other strategy that involves the conventional all-participate scheme. They also proposed a decentralized relay selection algorithm, where each relay node decides to relay or keep silent according to its own instantaneous channel gain and a predefined threshold. In [40], the proposed relay selection policy uses the available 
partial channel state information (CSI) at the source and relays. The policy selects an optimal relay which is the one with the maximum instantaneous scaled harmonic mean function of its source-relay and relay-destination channel gains. In [41], relay assignment problem in multi-user system was addressed. The proposed assignment policy is a practical policy for implementing cooperative diversity protocols to extend the coverage area. In [46-49], stochastic control of wireless cooperative networks is discussed. In [46], throughput optimal network control policies for single-source single destination cooperative networks are studied. The optimal policy takes into account the queue dynamics and it jointly optimizes routing, scheduling and resource allocation in the network. The work in [47] considers relay selection problem in cooperative networks from the stochastic control point of view. The proposed policy incorporates adaptive modulation and coding, as well as residual relay energy in the relay selection process to maximize the network life time. The authors in [47] formulate the relay selection problem in wireless cooperative networks as a stochastic control problem in a discounted Markov decision chain. The work in [49] investigates the optimal resource allocation policy for delay limited cooperative communication networks and develop dynamic cooperation strategies to achieve a target outage probability. In [48], the problem of throughput optimal relay selection in multi-user cooperative relaying access networks was addressed.

\subsection{Security Challenges in Cooperative Communica- tion}

Cooperative communication leads in significant performance improvements. But, since the source has to rely on an intermediate node, i.e., relay, security issues are brought up. Cooperative communication design is based on the assumption that the relay node always intend to help each other and cooperate effectively when they are selected to do so. However, this assumption may not hold when a node exists in the network which might misbehave for selfish or malicious intentions. A misbehaving node whether it is serving as a relay, source or destination can ruin the performance of the system. Therefore, security issues in cooperative communication are similar to the security concerns in other scenarios that also require collaboration among the entities in the network.

Cooperative communication can work efficiently and correctly based on the good 
behaviour assumption on the network entities. Particularly, it is assumed that they are willing to obey the policy and perform what they are expected to do. Otherwise, the misbehaving nodes can severely degrade the efficiency and performance of the system. For instance, it is possible for malicious nodes to join the network as relay and relay unwanted or unsolicited messages to the destination and hence degrading the performance of the system. A relay node which violates the cooperation strategy with the objective of disrupting communication between source and destination at the expense of consuming its own power and resources is characterized by having a malicious behaviour. A malicious node may refuse to forward the received packets when it is selected for cooperation and asked to forward the message. It may keep those packets in its queue so that the chance of assigning a channel to it for transmission increases. In many ways, a malicious node can attempt to ruin the performance of the system by misbehaving operations. In addition, some nodes may act in a selfish way to maintain their own energy or not to cooperate and make the source transmit its information through other relay nodes nodes thereby discouraging cooperation. There should be mechanisms to detect and resolve these issues. Otherwise, cooperative communication may suffer from severe performance degradation and discouraged cooperation.

While a selfish node is defined as a node who tries to use the network resources with out cooperating with other nodes or network controller, it will turn out that selfishness can severely affect the performance of the system. Hence, in this thesis, we define maliciousness as the act of refusing to cooperate with other entities in the network including the network controller where in it blocks or drops the received packet when it is expected to forward the received packet. It can be either due to the nodes' selfishness or the result of being compromised.

The following properties of cooperative communication causes vulnerability to attacks resulting in poor and disrupted cooperation of nodes in the network as can be seen in [26]:

- Possible Free-riders: as mentioned in this section, one of the main assumptions enabling the cooperative wireless network to work properly is that nodes are willing to share their resources among other entities in the network. As such, they have to cooperate with other nodes when they are expected and selected to do so. But, there is no assurance that assures the cooperation of the entities. In fact, a node may deliberately refuse to cooperate and relay a node's information. 
In this regard, an incentive may be considered for well-behaved nodes. To discourage the nodes which do not cooperate, well-behaved nodes can refuse to cooperate with a node without the assurance stating that the node can be trusted in terms of reciprocation.

- Absence of Centralized Control: nodes can move from area to area, from network to network and make relationships which may be short term. It is not feasible to get the complete knowledge of these relationships in advance. Therefore, by authentication, only those legitimate nodes must be allowed entering the network. Also, there should be a mechanism for dynamic monitoring of the nodes behavior.

- Frequent Topology Changes: possible changes in network topologies makes it difficult to prevent misbehaviour of relays. All nodes involved in cooperative relaying networks must be reliable to provide successful transmission between source and destination. There is no fixed shape of topology to the network and the topology could change frequently.

- Nature of Wireless Medium: nature of wireless medium causes vulnerability in the way that it is open to any access. In fact, there is no limitation in physical access and therefore, all nodes should be authenticated and monitored in various aspects.

- Resource Constraints: limited power resources and limited orthogonal relaying channels are two examples of the resource constraints in cooperative wireless networks. Wasting power and other resources is considered a misbehaviour in this type of network. In this environment it will not be easy to implement high complexity securing solutions.

- Scalability: any policy or protocol should be compatible to the inherent dynamic nature of the incoming relays and should be extensible in the network.

In this thesis, the trust establishment method we propose in Chapter 3 deals with mitigating the effects of frequently topology changes, absence of centralized control and resource constraints and distinguishing the selfish nodes.

Thus, it is acknowledged that security is one of the main concerns for the spread of cooperative communication and its adoption. Security involves the main goals 
of confidentiality, i.e., protecting the information, integrity, i.e., any change in the information should be done only by authorized entities and authorized mechanisms, and availability, i.e., information is available and accessible to authorized entities [50]. The attributes of a security concerns related to the above security goals as defined by the International Telecommunications Union - Telecommunication Standardization Sector (ITU-T) is given below [50-52]:

- Data Confidentiality: to make sure that the data can not be accessed by unauthorized users or nodes who are not the designated recipients, which could be generally achieved through cryptography.

- Data Integrity: to assure that the data has not been modified during the transmission process, which could be generally achieved through the use of hash functions.

- Authentication: to prevent any impersonation of a user or node in a network and to assist in correcting the identification of an entity.

- Non-repudiation: to make sure entities admit the ownership of their messages which be performed by verifying that the messages were sent with the node's credentials, which could be usually achieved through public key cryptography.

- Access Control: to provide protection against unauthorized access to data and to ensure that legitimate entities access the network.

Different security mechanisms have been established in the literature. Some of them are capable of performing counter malicious attacks to discourage the malicious or misbehavior. Usually, these mechanisms can be classified as the following categories based upon their application mode. Preventive and reactive mechanisms, or as preventıon-based and detection-based techniques are two types of classification of these mechanisms defined on the basis of their timing approach and the specific area of their targeted application. In the following, a brief description of each mechanism is provided.

The proposed method in Chapter 3 enables the system to distinguish the malicious nodes where apparently they pass all the properties above but still may become a compromised or selfish node by disobeying the routing rules. This will further deteriorate the performance of the system in terms of wasting the resources causing noticeable delays. 
- Preventive Mechanism: the goal is to employ some prevention techniques such as authentication, particularly data origin authentication, and integrity protection by encryption. In fact, these types of tools are the security mechanisms that act at the front line of defense against security attacks in cooperative communication networks.

- Reactive Mechanism: this operates as the front line of defense against security attacks and misbehavior that had bypassed the initial preventive mechanism.

- Prevention: It is the front line defense and works to avoid any attacks or unauthorized actions by malicious or adversarial nodes.

- Detection: to ensure that any adversarial, or misbehaving node that had entered the network can be traced and separated from the network.

\subsection{The Concept of Trust and Trust Management}

In various areas of science different definitions for trust has been appeared. For our purposes, trust level can be defined as the degree of belief about the behaviour of another entity [53]. The trust level associated with an entity may be interpreted in two different ways. Trust refers to subjective aspect of the trust level while trustworthiness refers to the objective aspect of the trust level. In general, trust is the probability of the event that an entity behaves as expected. As stated in [54], if the trustor ignores the gap between trustworthiness and trust, there will be a miscalculation of the involved risk. By misplacing trust, either the trustor looses the opportunity to cooperate with the trustees or the risk of deceit increases. Thus, we should minimize the difference between the calculated trust and the trustworthiness.

Trust management is the process of collecting the information necessary to establish a trust value and dynamically monitoring and adjusting the existing trust value. By using trust management, we can detect and decrease nodes' selfish or malicious misbehavior, e.g., dropping or refusing to forward packets for other nodes to save its battery power while still requiring other nodes' services. Few trust management surveys exist in the literature, such as $[54,55]$. [55] elaborates on various trust management methodologies as an action which can help minimize risk and assure the activity of benign entities in distributed systems. In [54], a research on trust management in Mobile Ad Hoc Networks MANETs) has been discussed. As it indicates, 
trust in MANETs has the following properties of subjectivity, dynamicity, asymmetry, incomplete transitivity and context dependency. Since Wireless cooperative networks have similar characteristics, the assumptions are also valid for wireless cooperative networks.

- Dynamicity: trust establishment in cooperative wireless relaying networks has to be based upon temporally and spatially local information

- Subjectivity: a trustor entity may derive a different level of trust against one particular node over time due to various experiences with the node as the topology changes.

- Incomplete Transitivity: trust is not necessarily transitive [56]. For instance, if A trusts $\mathrm{B}$, and $\mathrm{B}$ trusts $\mathrm{C}$, there is no assurance that A trusts $\mathrm{C}$. This is the case for more than two hop relaying networks or multi-user systems.

- Asymmetry: trust is asymmetric, not necessarily reciprocal. For instance, node A trust B but, node B does not trust A.

- Context Dependency: trust is context-dependent, for example node A may trust $B$ in one regard but not another one. Based upon the application, different types of trust should be considered, e.g., trust in computational power, trust in forwarding or trust in reporting.

For our purpose in cooperative wireless mobile relaying networks, we expect a relay node to forward the received packet to destination whenever it is selected to do so and the link between relay and destination has an acceptable quality of channel. Similarly, in the systems with combining at the destination, if the combined SNR is greater than a threshold and the relay has been selected to forward the received packet, it is expected to do so. Note that the destination does not receive the information correctly if the combined SNR is less than a threshold while using maximum ratio combining at the destination [11]. Having defined the network expectation from a relay, henceforth, we use trust and trustworthiness interchangeably, and we will refer to such misbehaving relay nodes as malicious nodes. Misbehaviour of malicious relays may deteriorate the performance of the system severely. This motivates us to look for a mechanism which can distinguish the misbehaving relay nodes from the benign ones. 
To this end, each relay node is associated with a real value from the interval $[0,1]$ which is called trust representing to what extent it is trustworthy. In general, trust is defined as the probability that the trustee performs a specific action expected of it [4]. In cooperative wireless systems, each relay node is a trustee which is expected to forward the received packet to the destination when it is selected for cooperation. Trust establishment is a scheme to evaluate and assign a trust value to every entity in the network which enables the trustor (e.g., the source) to detect misbehaving nodes.

A few studies have been conducted on security issues about security issues in cooperative communication which most of them are dealing with detection based techniques. $[57,58]$ propose a detectıon-based technique which traces the adversarial nodes who transmit unsolicited messages to the destination by using adaptive signal detection at the physical layer while employing pseudorandom tracing symbols in the application layer level. In [59], the authors propose a scheme for cooperative jamming to assure the security of the communication using an untrusted relay in which the destination or another node jams the relay and uses the jamming message signal as the side information. In [60], the authors consider information-theoretically secure communication between two special nodes and establish a cooperative secrecy set-up. In [61], a noise-forwarding strategy is established enforcing the relays to act as trusted third parties by sending codewords independent of the message sent by the source for the purpose of confusing the eavesdropper. An approach to detect selfishness and enforce distributed cooperation based on monitoring neighbors to identify a misbehaving node that does not cooperate during data transmission is proposed in [62]. In [63] and [64], an additional punishment mechanism to isolate the misbehaving and non-cooperating nodes has been proposed. In [65], the authors propose a security system developing a trust model, assigning credentials to nodes, updating private keys, managing each node's trust value, and making appropriate decisions about nodes' access rights. [66] combines AF, DF, and cooperative jamming and the preceding outlined concepts above to propose a security scheme. [67] proposes two new security schemes in the media access control layer to address the security concerns arising in cooperative communication with emphasis on IEEE 802.11 architecture. A statistical detection technique is proposed in [68] to mitigate malicious behavior by having the destination examine the relay's signal before to applying diversity combining with the direct signal from the source.

A large body of research has focused on trust establishment in MANETs [5,69-72]. 
In [69], the authors propose a trust establishment method with high adaptability for MANETs based on game theory. Authors in [70] elaborate upon issues related to trust and introduce a context-aware reputation based method of trust establishment. In [71], a secure authentication approach for multicast MANETs is proposed, employing a Markov chain trust model to determine the trust value for each one-hop neighbour.

Bayesian methodology is a well known approach for trust establishment $[73,74]$. It is widely used for evaluating the nodes' trust in wireless networks specifically in $[5,72,75]$. In Bayesian methodology, under the assumption that every transmission is independent of previous transmissions, trust values are derived and follow a Beta distribution. Parameters of Beta distribution are obtained by performing repeated observations. In [72], Bayesian methodology is used to compute a trust value and a distributed reputation system is constructed. The objective in [5] is to achieve a trust establishment method in order to have reliable data packet delivery. The approach is based on Bayesian methodology using trust and confidence that are computed to construct a reputation model. In [75], the authors design a trust-assisted cooperative transmission scheme. The trust model they employed is based on Bayesian methodology similar to the work in [5]. In their approach, maliciousness and unreliability of channels are taken into consideration simultaneously for signal combining at the destination.

In the next chapter, we design a trust establishment scheme that takes into account the relay selection policy and channel condition information in the trust computation. This trust establishment method can be applied to any system with a general relay selection policy whose decisions in each cooperative transmission are independent of the previous ones. The framework which is further used in the proposed trust establishment is Bayesian methodology which is reviewed in the next section in detail.

\subsubsection{Bayesian Methodology}

The Bayesian methodology is an approach that is well-suited for stochastic problems of the type we wish to address. The following is review of this methodology along with an example of its application. In section 3.3 , we will demonstrate that this methodology needs to be extended to be applied effectively to our security model. Consider a single node $A$ acting as a relay node in a network. Node $A$ receives data packets and then forwards them to a downstream receiver. If node $A$ behaves maliciously, it discards some of the received packets from its queue and does not forward 
them. If we model the maliciousness by a Bernoulli random variable with parameter $q, A$ discards a packet with probability $q$ independent of other transmissions. To such a node, a trust value $T$ from the interval $[0,1]$ is associated which is an estimate of the parameter $\bar{q}=1-q$, i.e., the unknown parameter to the trustor. In fact, the trustor summarizes its belief about $\bar{q}$ in a probability distribution assuming that $\bar{q}$ follows a prior distribution. It then updates this distribution by performing iterative observations on the system, i.e., counting the number of packets entering node $A$ and the number of forwarded packets by $A$. At the beginning, when there is no observation data about the system available, the trustor assumes that $\bar{q}$ follows a uniform distribution (the prior distribution at $t=0$ ). Each time the trustor makes an observation, say at iteration $t, t \in \mathbb{N}$, it can derive the posterior distribution of $\bar{q}$ at that time based on the prior distribution of $\bar{q}$ at $t-1$ and the current observation at iteration $t$. It then, uses the obtained posterior distribution as the prior distribution at iteration $t+1$ and this procedure continues. The expected value of $\bar{q}$ with respect to the obtained posterior distribution after observation $t$ is called the trust value at time $t[5,72-74]$.

Suppose that we observe the system iteratively and during each iteration $t$ we observe $k(t)$ packets received by $A$ and $\ell(t)$ packets transmitted successfully by $A$. Also, assume that the trustor can summarize its belief about the random variable $\bar{q}$ in a prior distribution $f_{t-1}(\bar{q})$. Let $L(t)$ denote the random variable corresponding to number of packets transmitted successfully by $A$. Having $k(t), \ell(t)$ and $f_{t-1}(\bar{q})$, the trustor can derive the posterior distribution of random variable $\bar{q}$ via Bayes' rule as follows.

$$
f_{t}(\bar{q})=\frac{P(L(t)=\ell(t) \mid \bar{q}, K(t)=k(t)) f_{t-1}(\bar{q})}{\int_{0}^{1} P(L(t)=\ell(t) \mid \bar{q}, K(t)=k(t)) f_{t-1}(\bar{q}) d \bar{q}}
$$

Note that, $P(L(t)=\ell(t) \mid \bar{q}, K(t)=k(t))$ follows a binomial distribution, i.e.,

$$
P(L(t)=\ell(t) \mid \bar{q}, K(t)=k(t))=\left(\begin{array}{c}
k(t) \\
\ell(t)
\end{array}\right) \bar{q}^{\ell(t)} q^{k(t)-\ell(t)} .
$$

As Binomial distribution and Beta distribution are a conjugate pair, one can easily derive the distribution function of $\bar{q}$ as follows. At the beginning (iteration 0 ), we have no information about distribution of $\bar{q}$. Therefore, we assume that $\bar{q}$ follows a 
uniform distribution

$$
f_{0}(\bar{q})=U(0,1)=\operatorname{Beta}(1,1)
$$

where

$$
B e t a(\alpha, \beta)=\frac{\bar{q}^{(\alpha-1)} q^{(\beta-1)}}{\int_{0}^{1} \bar{q}^{(\alpha-1)} q^{(\beta-1)} d \bar{q}}
$$

It can be easily shown that if $f_{t-1}(\bar{q})=\operatorname{Beta}(\alpha(t-1), \beta(t-1))$, given $K(t)=k(t)$ and $L(t)=\ell(t)$, we have

$$
f_{t}(\bar{q})=\operatorname{Beta}(\alpha(t-1)+\ell(t), \beta(t-1)+k(t)-\ell(t)), \quad t \geq 1
$$

In other words, $f_{t}(\bar{q})$ follows a $\operatorname{Bet} a(\alpha(t), \beta(t))$ distribution with the parameters

$$
\alpha(t)=\alpha(t-1)+\ell(t), \alpha(0)=1, \quad \beta(t)=\beta(t-1)+k(t)-\ell(t), \beta(0)=1
$$

Note that equations (2.3) and (2.5) construct a recursive formula by which we can easily update the prior distribution function $f_{t}(\bar{q})$ without any need to calculate (2.1) from scratch.

Based on the previous discussion, after the $t$-th iteration, trust value would be the expected value of $\bar{q}$ with respect to the distribution function $f_{t}(\bar{q})$, i.e.,

$$
T(t)=\frac{\alpha(t)}{\alpha(t)+\beta(t)}
$$

It is worth mentioning that more than one stream of data may be passed through node $A$ from different upstream traffic sources. Therefore, each of these upstream sources may observe different observation about the malicious behavior of $A$. In such case, a weighted sum of other users opinion about the trust value of $A$ can be an estimate of $A$ 's trust value. It is also possible that in a multi-user system, a relay behaves differently with regard to different streams originating from different users. In this case, one possible approach is to derive a trust value for every pair of user and relay. One of our experiments in section 4.3 clarifies this issue in a multi-user system. In this section we just talked about one stream of data passing through node $A$.

The Bayesian framework described above is a general framework to estimate or 
derive a distribution of the unknown parameter of a process by using observations $[73,74]$. In fact, if we can model a process in a system with a Bernoulli process (independent successes and failures) with unknown probability of success or failure, Bayesian methodology can be used to obtain an estimate of the unknown probability as the mean of the Beta distribution function.

\subsection{Summary}

In this chapter, the background knowledge and related work of this thesis was presented by looking at the fundamentals of cooperative communication, cooperative relaying and relay selection, security issues in cooperative communication, trust and trust management and Bayesian methodology. Due to the inherent nature of the cooperative networks with the use of any available intermediate nodes as relays, a dynamic relay selection processes trust establishment are required beside authentication and cryptographic techniques. To this end, relay selection in cooperative wireless relaying networks and Bayesian methodology were described. In the following chapter, we will see the proposed trust establishment method in detail. 


\section{Chapter 3}

\section{Trust Establishment in Cooperative Wireless Networks}

\subsection{Introduction}

In cooperative wireless networks, relay nodes are used to improve the channel capacity of the system. However, the presence of misbehaving relays in the network may severely deteriorate the performance of the system. For instance, there exists a possibility that a node refuses to cooperate when it is selected for cooperation or deliberately drop the received packets. Trust establishment is a mechanism to detect misbehaving nodes in a network. Although authentication and cryptographic techniques provide promising approaches to make the system secure and provide confidentiality and integrity, there still remains the chance for compromised relay nodes to take part in the cooperation process and disrupt the transmissions. Another example is the case where a selfish (and not necessarily compromised) relay node refuses to cooperate or deliberately drop the received packets (for different reasons e.g. power saving) when it is selected for cooperation. Henceforth, we will refer to such misbehaving relay nodes as malicious nodes. Misbehaviour of malicious relays may degrade the performance of the system severely. This motivates us to look for a mechanism which can distinguish the misbehaving relay nodes from the benign ones. To this end, each relay node is associated with a real value from the interval $[0,1]$ which is called trust representing to what extent it is trustworthy. In cooperative wireless relaying systems, each relay node is a trustee with regarding to relaying operation and is further expected to forward the received packet to the destination when it is selected for cooperation. Trust establishment is a scheme to evaluate and assign a 
trust value to every entity in the network which enables the trustor (e.g., the source) to detect misbehaving nodes. In the Bayesian methodology under the assumption that every transmission is independent of previous transmissions, trust values are derived and follow a Beta distribution. Parameters of Beta distribution are obtained by performing repeated observations. In this chapter, we propose a trust establishment method for cooperative wireless relaying networks using Bayesian framework reviewed in section 2.3.

In contrast with previous schemes, this approach takes channel state information and the relay selection policy into account to derive a pure trust value for each relay node. The trust of a given relay node will decrease if and only if it misuses the opportunity of cooperative transmission or refuse to cooperate when it is selected for cooperation. The considered model is a two phase cooperation protocol where in the first phase the source node broadcasts the information to the relay nodes as well as the destination. In the second phase, one of the relay nodes is selected for cooperation according to the employed relay selection policy and transmits the information to the destination. The destination node, then combines the signals received in the the first and second phases and detects the information from the combined signal. The proposed method can be applied to any system with a general relay selection policy whose decisions in each cooperative transmission are independent of the previous ones. It is worth mentioning that, the method does not impose additional communication overhead on the system as it uses the available information in the relay selection procedure. The results of the work in this chapter has been published in [76, 77].

The rest of this chapter is organized as follows. In section 3.2, we will describe the model used in this chapter. In section 3.3, we will see how using Bayesian methodology in wireless cooperative networks is insufficient in terms of trust establishment by two examples. In section 3.3.1, we will introduce the proposed trust establishment schemes for cooperative relaying networks. Simulation results are brought in section 3.4. Section 3.5 summarizes the conclusions.

\subsection{Model Description}

The model that we consider consists of a single source node $S$, a destination node $D$, and a set of relay nodes $\mathcal{R}=\left\{r_{1}, r_{2}, \ldots, r_{R}\right\}$ where $|\mathcal{R}|=R$. The relay nodes are 
pure relay stations and do not inject traffic to the system. All nodes operate in halfduplex mode, i.e., they cannot transmit and receive the information simultaneously. The transmission paradigm from $S$ to $D$ is a two-phase transmission protocol which is widely used in cooperative wireless networks such as $[8,9,78,79]$. In the first phase of communication, $S$ broadcasts a data packet to all relay nodes using a fixed amount of power $P_{s}$ while the relay nodes and destination are listening. In the second phase, one of the relay nodes which has successfully received the packet will be selected to forward the packet to $D$. We call the two phases of transmission of a packet a transmission frame. The relay nodes work in DF (Decode and Forward) mode. All transmissions from each relay $i$ use a fixed amount of power $P_{\imath}$. All links in the model are assumed to be slow Rayleigh fading channels with additive white Gaussian noise (AWGN). Based on the channel quality of the direct link from $S$ to $D$, we may consider two cases. In the first case, the channel quality between $S$ and $D$ is poor. Therefore, destination does not keep the received signal transmitted by source in the first phase for the purpose of combining. Therefore, if we denote the transmitted signal from the source in the first phase by $X_{S}$, received signal at each relay $i$ would be

$$
Y_{r_{\imath}}=\frac{h_{S-r_{2}}}{d_{S-r_{\imath}}^{\frac{a}{2}}} X_{S}+Z_{\imath}, \quad \forall i=1, \ldots, R
$$

where $h_{S-r_{2}}$ is the Rayleigh channel coefficient, $d_{S-r_{z}}$ is the distance between the source $S$ and the relay node $r_{\imath}, a$ is the path loss exponent and $Z_{\imath}$ denotes the additive zero mean Gaussian noise at relay $i$. Similarly, if $X_{2}$ denotes the transmitted signal from relay $i$ in the second phase, the received signal at the destination is

$$
Y_{D}=\frac{h_{r_{\imath}-D}}{d_{r_{\imath}-D}^{\frac{2}{2}}} X_{\imath}+Z_{D}, \quad \forall i=1, \ldots, R
$$

In the second case, the direct link between $S$ and $D$ has an acceptable quality of channel. Despite the first case, $D$ always keeps the received signal in the first phase of transmission to perform combining at the end of second time slot. Other settings discussed above for the first case of the model are valid for the second case. Note that, received signal at the destination in the first and second phase of transmission are the following, 


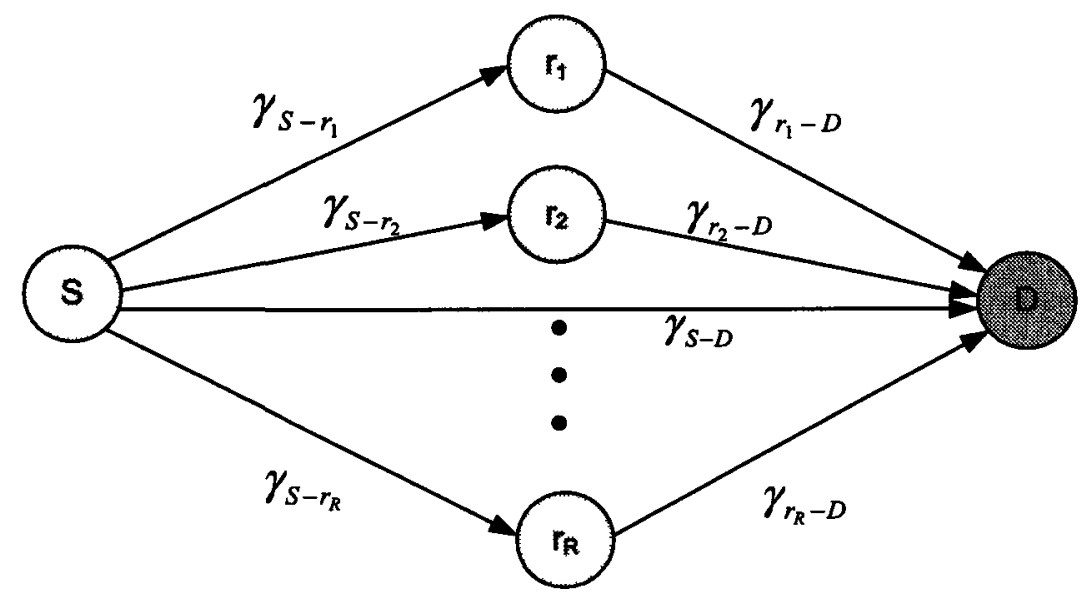

Figure 3.1: The cooperative communication model.

$$
\begin{gathered}
Y_{1 D}=\frac{h_{S-D}}{d_{S-D}^{\frac{a}{2}}} X_{S}+Z_{D}, \\
Y_{2 D}=\frac{h_{r_{2}-D}}{d_{r_{\imath}-D}^{\frac{a}{2}}} X_{\imath}+Z_{D}, \quad \forall i=1, \ldots, R
\end{gathered}
$$

where $h_{S-D}$ and $h_{r_{2}-D}$ are fading coefficients of link $S-D$ and $r_{2}-D$ respectively. $d_{S-D}$ and $d_{r_{\imath}-D}$ represent the distance from $S$ to $D$ and from relay $\imath$ to $D$ respectively. The model employs a relay selection policy, which selects an appropriate relay node based on the available instantaneous channel state information (CSI) of the system. We will assume that each relay selection is performed independent of the previous or future relay selections or channel states. Although this assumption seems to be restrictive, such a class of policies are discussed and have been used in cooperative communication research $[78,79]$. In this section, we will use the maximum SNR policy as the relay selection policy, similar to the approach which is used in [78,79]. Although we use a specific policy in our analysis and main part of simulations, the results extend to any policy with the above property, i.e., independence of relay selections from past and future relay selections. However, in an experiment in the simulations we will show the result of applying the proposed trust establishment method in a system using a different policy. In the following, we will describe the employed relay selection policy in more detail. 
Let us denote the instantaneous SNR of the $S-r_{\imath}, r_{\imath}-D$ and $S-D$ links by $\gamma_{S-r_{2}}$, $\gamma_{r_{2}-D}$ and $\gamma_{S-D}$ respectively. We assume that the noise power in the transmission spectrum is $W$. Based on the previous discussion and assuming, we have the following equations for instantaneous SNRs.

$$
\gamma_{S-r_{2}}=\frac{\left|h_{S-r_{2}}\right|^{2} P_{S}}{d_{S-r_{\imath}}^{a} W}, \gamma_{r_{2}-D}=\frac{\left|h_{r_{2}-D}\right|^{2} P_{2}}{d_{r_{2}-D}^{a} W}, \gamma_{S-D}=\frac{\left|h_{S-D}\right|^{2} P_{S}}{d_{S-D}^{a} W}
$$

Figure 3.1 shows the communication model used in this chapter consisting of source $S$, Destination $D$ and $R$ relay nodes in between.

As mentioned earlier, during the first phase of communication, the source $S$ broadcasts a packet to the relays. Among the relays, those who have SNR greater than a threshold SNR, $\gamma_{t h r}$, can decode the signal correctly. The value of $\gamma_{t h r}$ depends on the acceptable BER for the application running on the network and also the modulation and coding schemes used. For example, for voice and video the acceptable $\operatorname{BER}\left(10^{-3}-10^{-6}\right)$ is different than what is needed for file transfer $\left(10^{-6}-10^{-9}\right)$. The BER-SNR curve is also different for different modulation and coding scheme [11]. Thus, based on the application, the modulation and coding schemes, such a threshold SNR can be determined. Here, we assume that the system is using BPSK modulation without coding and the acceptable BER is $10^{-6}$. Suppose that if a relay node decodes a packet correctly, it broadcasts an instantaneous ACK to the destination. Let $\Omega$ represent the set of relay nodes who have decoded the packet correctly. Afterwards, the destination (who is responsible for selecting an appropriate relay node) chooses a relay node whose $\gamma_{r_{2}-D}$ is the maximum, i.e.,

$$
r^{*}=\arg \max _{r_{2} \in \Omega} \gamma_{r_{i}-D}
$$

where $r^{*}$ denotes the selected relay node. $r^{*}$ then transmits the packet to node $D$. For detection at $D$ in the case without combining (case 1), D simply decodes $Y_{D}$. In the second case, $D$ will further use maximum ratio combining (MRC) at the destination to combine two received signals from $r^{*}$ and $S$, i.e., $Y_{1 D}$ and $Y_{2 D}$. [11]. If $D$ can decode the packet correctly, it broadcasts an instantaneous ACK and the source node erases the packet from its queue. Otherwise, it broadcasts a NACK and the source retransmits the packet in the next frame of transmission. We will model the maliciousness of each relay node $i$ by a binary random variable $Q_{\imath}$ with parameter $q_{2}$. That is, we assume that at each transmission any malicious relay node $i$ selected 
to relay a packet to the destination behaves anomalously and does not forward the packet with probability $q_{i}$. Stochastic modelling of the maliciousness of a node has appeared previously in the literature, e.g., $[5,72,80]$. In fact, a clever attacker may use stochastic malicious behaviour in which the malicious relay tries to mimic the inherent uncertainty of the wireless channel [80].

Our purpose is to derive a trust value corresponding to each relay node which is equal to $1-E\left[q_{2}\right]$. That is, the trust value is the complement of the expectation value of the maliciousness random variable. To this end, we perform iterative observations of the system including the channel conditions, relay selections and the net amount of packets received and forwarded by each relay node. Based on these observations we will derive the current trust value after each observation iteration.

\subsection{Trust Establishment For Wireless Cooperative Relaying Networks}

In the cooperative system described in section 3.2, not all of the unsuccessful packet transmissions from a relay node are due to maliciousness of that node. They may also be the result of unreliability of channels, packet overflow, etc. Therefore, using the Bayesian framework solely based on the number of received and successfully forwarded packets cannot result into an accurate derivation of trust value. In other words, the obtained trust value would not be a pure trust value and will be biased. The following example clarifies this fact.

Example: Consider a simple cooperative system with four nodes. The source node 1 generates traffic and sends the traffic to node 4 via cooperation with nodes 2 and 3. The transmission protocol is as discussed in section 3.2. The channel between the source node 1 and relay nodes 2 and 3 are perfect noise free channels. Among nodes 2 and 3 , one of them is chosen randomly (probabilities of 0.5 ) to forward the packet to the destination node 4 . The channel between relay node 2 and destination node 4 is modelled by a binary erasure channel with success probability of 0.95 and the channel between relay node 3 and destination node 4 is modelled by another binary erasure channel with success probability of 0.65 . Relay 2 is malicious with probability 0.35 and relay node 3 is malicious with probability 0.2. Using the Beta function trust value establishment discussed in the previous section, we obtained the trust value through simulations. We have assumed that each iteration consists of 100 transmission frames. 
We expect the trust of relay 2 and relay 3 to be 0.65 and 0.8 , respectively. As we can

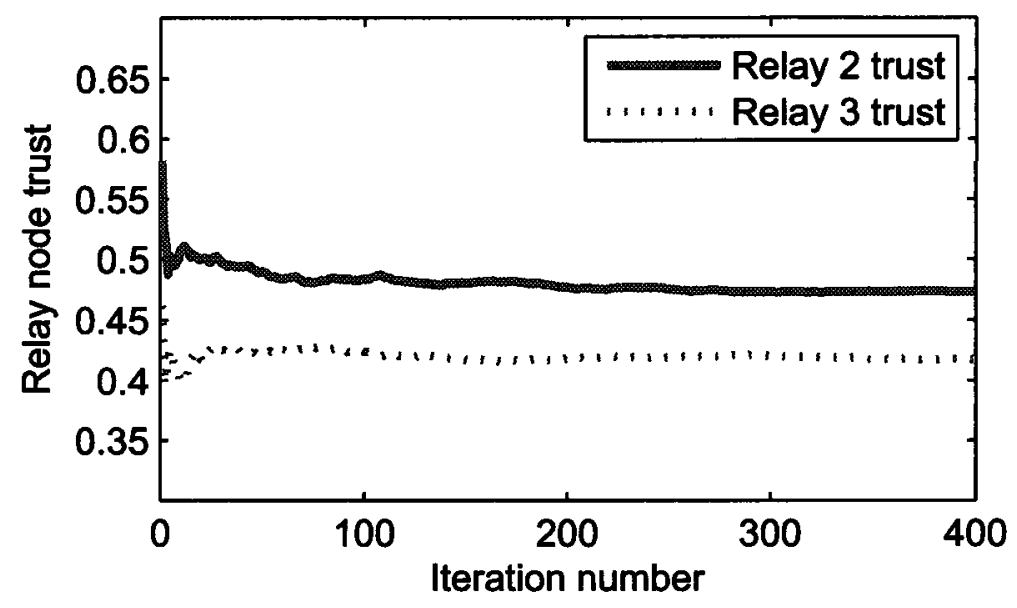

(a)

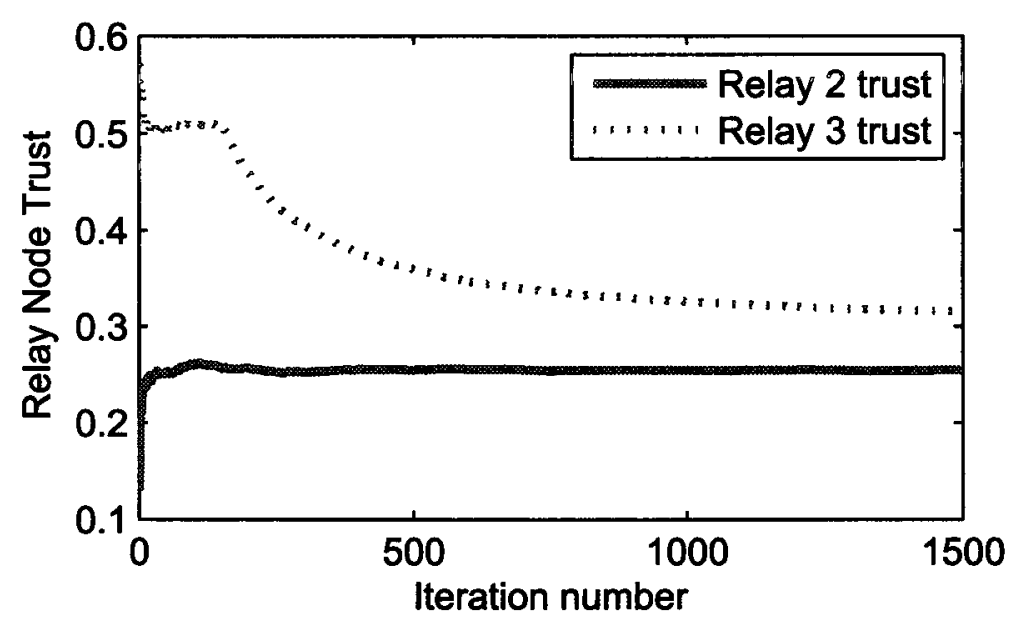

(b)

Figure 3.2: Conventional Bayesian trust establishment in a simple cooperative system.

see in the Figure 3.2.a, the conventional Bayesian trust establishment gives inaccurate trust values with inverse order rather than what we expect. That is, it gives 0.475 for relay 2 and 0.425 for relay 3 . Although relay 2 is more malicious than relay 3 , it has a larger connectivity probability and subsequently, more successfully transmitted packets than relay 3. Therefore, using conventional Bayesian methodology we achieve a larger trust value for relay 2 than for relay 3 . That is, the result is biased by the channel conditions. 
Consider another example where the success probability of channels between node 2 and 4 and between node 3 and 4 is 0.65 and 0.85 , respectively. Suppose that nodes 2 and 3 are initially malicious with probabilities 0.55 and 0.2 . After iteration 150, node 3 changes its maliciousness to 0.6 . Figure 3.2 .b shows the trust tracking of the system using the conventional Bayesian trust establishment. Before iteration 150, we expect the trust of relay 2 and relay 3 to be 0.45 and 0.8 , respectively but, it gives us inaccurate result of 0.25 and 0.5 , respectively. After the maliciousness of relay node 3 changes from 0.2 to 0.6 , the trust of relay 3 should change to 0.4 i.e., less than the trust of relay 2 which should now be 0.45 . Using the conventional Bayesian method, computed trusts at the end are 0.25 for relay 2 and 0.32 for relay 3. Importantly, this method gives us the inverse ranking of trusts at the end after changing the maliciousness of node 3 . In other words, although this method tries to track the trust after iteration 150, the starting and ending point of this tracking are not correct. Again, not considering the channel connectivity probabilities and relay selection probabilities in trust establishment is problematic.

We will see similar results for more realistic channel conditions and relay selection policy in section 3.4.

\subsubsection{Proposed Trust Establishment for Wireless Cooperative Relaying Networks}

Given the problems with the conventional Bayesian approach, we now introduce a trust establishment scheme for the model in two cases described in section 3.2 that considers the channel conditions. In our scheme, our aim is to obtain an accurate trust value corresponding to each relay node. In other words, the destination $D$ (e.g., a base station) which manages the relay selection in the system, should be able to obtain an accurate trust value for each relay node. The destination can then use these trust values and may combine them with the trust values obtained from other nodes to decide whether a relay node is trustworthy or not. It can later take the proper action or remove a relay node from its list of reliable relay nodes if its associated trust value drops below a specific threshold.

As explained earlier, trust establishment is usually based on iterative monitoring of the system and it involves many message exchanges between trustor and trustee. In our method we will make use of existing message exchanges in the relay selection and channel state estimation to our benefit. At each iteration $t$, the destination 
counts the number of successful transmissions from $S$ to each relay node $i$. It can count this number by using the number of ACKs broadcasted from relay $i$. Let $k_{\imath}(t)$ denote this number. The total number of successful transmissions from relay $i$ to $D$ is also counted. These numbers depend on the maliciousness of the relay node $i$ as well as channel conditions and the relay selection policy. Suppose that the destination observes $\ell_{\imath}(t)$ successful transmission from relay $i$ to $D$ at iteration $t$. The conventional Bayesian trust establishment computes the trust for relay node $i$ at iteration $t$ as follows.

$$
T_{\imath}(t)=\frac{\alpha_{\imath}(t)}{\alpha_{\imath}(t)+\beta_{\imath}(t)}, \quad \imath \in\{1,2, \ldots, R\}, t \in \mathbb{Z}^{+} .
$$

where

$$
\alpha_{\imath}(t)=\alpha_{\imath}(t-1)+\ell_{\imath}(t), \alpha_{\imath}(0)=1, \beta_{\imath}(t)=\beta_{\imath}(t-1)+k_{\imath}(t)-\ell_{\imath}(t), \beta_{\imath}(0)=1
$$

As discussed earlier, the amount of $\alpha_{\imath}(t)$ depends not only on the maliciousness of a relay node but also the number of times that the relay was selected for cooperation as well as channel conditions. Therefore, the obtained trust values in (3.7) are biased on the channel conditions and the relay selection policy. We attempt to remove this bias by modifying the trust value in (3.7). Let $\Theta_{2}$ represent the event that relay node $\imath$ is selected by the destination described in section 3.2, respectively. Therefore, for case 1 and case 2 , we can obtain the accurate trust values $T_{\imath}^{(1)}(t)$ and $T_{\imath}^{(2)}(t)$ after iteration $t$ as

$$
\begin{aligned}
T_{\imath}^{(1)}(t)=\frac{\alpha_{\imath}^{(1)}(t)}{\alpha_{\imath}^{(1)}(t)+\beta_{\imath}^{(1)}(t)} \cdot \frac{1}{P\left(\gamma_{r_{\imath}-D} \geq \gamma_{t h r}\right)} \cdot \frac{1}{P\left(\Theta_{\imath} \mid \gamma_{r_{\imath}-D} \geq \gamma_{t h r}, \gamma_{S-r_{\imath}} \geq \gamma_{t h r}\right)} \\
T_{\imath}^{(2)}(t)=\frac{\alpha_{\imath}^{(2)}(t)}{\alpha_{\imath}^{(2)}(t)+\beta_{\imath}^{(2)}(t)} \cdot \frac{1}{P\left(\gamma_{r_{\imath}-D}+\gamma_{S-D} \geq \gamma_{t h r}\right)} \\
\cdot \frac{1}{P\left(\Theta_{\imath} \mid \gamma_{r_{\imath}-D}+\gamma_{S-D} \geq \gamma_{t h r}, \gamma_{S-r_{2}} \geq \gamma_{t h r}\right)}
\end{aligned}
$$

Note that the destination does not know the statistics of relay selections and also the channel conditions. Therefore, the destination follows the same estimation approach to obtain the values for $P\left(\gamma_{r_{\imath}-D} \geq \gamma_{t h r}\right), P\left(\Theta_{\imath} \mid \gamma_{r_{\imath}-D} \geq \gamma_{t h r}, \gamma_{S-r_{\imath}} \geq \gamma_{t h r}\right)$, 
$P\left(\gamma_{r_{\imath}-D}+\gamma_{S-D} \geq \gamma_{t h r}\right)$ and $P\left(\Theta_{\imath} \mid \gamma_{r_{\imath}-D}+\gamma_{S-D} \geq \gamma_{t h r}, \gamma_{S-r_{\imath}} \geq \gamma_{t h r}\right)$. In other words, given the independence of relay selection decisions in different frames, we can model the selection of a relay $i$ given the events $\gamma_{S-r_{2}} \geq \gamma_{t h r}$ and $\gamma_{r_{2}-D} \geq \gamma_{t h r}$ in case 1 and the selection of a relay $i$, given the events $\gamma_{S-r_{z}} \geq \gamma_{t h r}$ and $\gamma_{r_{2}-D}+\gamma_{S-D} \geq \gamma_{t h r}$ in case 2 as two Bernoulli random variables with parameter $p_{\Theta_{\imath}}^{(1)}$ and $p_{\Theta_{i}}^{(2)}$, respectively. Assume that $\ell_{\Theta_{2}}^{(1)}(t)$ in case 1 and $\ell_{\Theta_{1}}^{(2)}(t)$ in case 2 represent the number of times that relay $i$ is selected in iteration $t$ given that both $\gamma_{S-r_{i}}$ and $\gamma_{r_{2}-D}$ in case 1 and both $\gamma_{S-r_{2}}$ and $\gamma_{r_{2}-D}+\gamma_{S-D}$ in case 2 are greater that the threshold SNR. Given $\ell_{\Theta_{2}}^{(1)}(t), \ell_{\Theta_{2}}^{(2)}(t)$ and $k_{\imath}(t)$, the parameter $p_{\Theta_{\imath}}^{(1)}$ in case 1 follows a Beta distribution of $\operatorname{Beta}\left(\alpha_{\Theta_{\imath}}^{(1)}(t), \beta_{\Theta_{\imath}}^{(1)}(t)\right)$ and $p_{\Theta_{\imath}}^{(2)}$ in case 2 follows the distribution of $\operatorname{Beta}\left(\alpha_{\Theta_{\imath}}^{(2)}(t), \beta_{\Theta_{\imath}}^{(2)}(t)\right)$ at iteration $t$ and its corresponding mean is the following,

$$
\frac{\alpha_{\Theta_{\imath}}^{(J)}(t)}{\alpha_{\Theta_{\imath}}^{(J)}(t)+\beta_{\Theta_{\imath}}^{(J)}(t)} j=1,2
$$

where

$$
\begin{gathered}
\alpha_{\Theta_{\imath}}^{(\jmath)}(t)=\alpha_{\Theta_{\imath}}^{(\jmath)}(t-1)+\ell_{\Theta_{\imath}}^{(\jmath)}(t), \quad \beta_{\Theta_{\imath}}^{(\jmath)}(t)=\beta_{\Theta_{\imath}}^{(\jmath)}(t-1)+k_{\imath}(t)-\ell_{\Theta_{\imath}}^{(\jmath)}(t) \\
\alpha_{\Theta_{\imath}}^{(\jmath)}(0)=1, \quad \beta_{\Theta_{\imath}}^{(\jmath)}(0)=1, \quad \text { for } j=1,2
\end{gathered}
$$

In the above equations, $j=1$ belongs to case 1 and $\jmath=2$ is for case 2 . We will further use this value as an estimate of $P^{(1)}\left(\Theta_{\imath} \mid \gamma_{r_{\imath}-D} \geq \gamma_{t h r}, \gamma_{S-r_{\imath}} \geq \gamma_{t h r}\right)$ in case 1 and $P^{(2)}\left(\Theta_{\imath} \mid \gamma_{r_{\imath}-D}+\gamma_{S-D} \geq \gamma_{t h r}, \gamma_{S-r_{\imath}} \geq \gamma_{t h r}\right)$ in case 2. The same argument is used to estimate the $P\left(\gamma_{r_{2}-D} \geq \gamma_{t h r}\right)$ and $P\left(\gamma_{r_{2}-D}+\gamma_{S-D} \geq \gamma_{t h r}\right)$, i.e., given the independence of channel states in different frames, these two events are modelled by two Bernoulli random variables whose parameters $p_{\gamma_{i}}^{(1)}$ and $p_{\gamma_{2}}^{(2)}$ respectively follow Beta distribution at each iteration. Specifically, in the case 1 , if the $D$ observes that the SNR of the link $r_{\imath}-D$ is greater than the threshold in $\ell_{\gamma_{z}}^{(1)}(t)$ cases out of $k_{\imath}(t)$ and in case 2, if it observes that $\gamma_{r_{2}-D}+\gamma_{S-D} \geq \gamma_{t h r}$ in $\ell_{\gamma_{2}}^{(2)}(t)$, then $p_{\gamma_{2}}^{(\jmath)}$ given $\ell_{\gamma_{2}}^{(\jmath)}(t)$ and $k_{\imath}(t)$ follows a Beta distribution $\operatorname{Beta}\left(\alpha_{\gamma_{2}}^{(j)}, \beta_{\gamma_{2}}^{(j)}\right)$ where

$$
\alpha_{\gamma_{2}}^{(\jmath)}(t)=\alpha_{\gamma_{2}}^{(\jmath)}(t-1)+\ell_{\gamma_{2}}^{(\jmath)}(t), \quad \beta_{\gamma_{2}}^{(\jmath)}(t)=\beta_{\gamma_{2}}^{(\jmath)}(t-1)+k_{\imath}(t)-\ell_{\gamma_{2}}^{(\jmath)}(t)
$$




$$
\alpha_{\gamma_{2}}^{(\jmath)}(0)=1, \quad \beta_{\gamma_{2}}^{(\jmath)}(0)=1 \text { for } j=1,2
$$

with mean $\frac{\alpha_{\gamma_{2}}^{(j)}(t)}{\alpha_{\gamma_{2}}^{(g)}(t)+\beta_{\gamma_{2}}^{(g)}(t)}$. We use this value as the estimate of $P\left(\gamma_{r_{2}-D} \geq \gamma_{t h r}\right)$ for case 1 and $P\left(\gamma_{r_{i}-D}+\gamma_{S-D} \geq \gamma_{t h r}\right)$ for case 2 at iteration $t$.

Using the same approach, we can then formulate the trust at each iteration $t$ as

$$
T_{\imath}^{(\jmath)}(t)=\frac{\alpha_{2}^{(\jmath)}(t)}{\alpha_{\imath}^{(\jmath)}(t)+\beta_{\imath}^{(\jmath)}(t)} \cdot \frac{\alpha_{\Theta_{2}}^{(\jmath)}(t)+\beta_{\Theta_{\imath}}^{(\jmath)}(t)}{\alpha_{\Theta_{\imath}}^{(\jmath)}(t)} \cdot \frac{\alpha_{\gamma_{2}}^{(\jmath)}(t)+\beta_{\gamma_{2}}^{(\jmath)}(t)}{\alpha_{\gamma_{2}}^{(\jmath)}(t)}
$$

where the parameters $\alpha_{\imath}^{(\jmath)}(t), \beta_{\imath}^{(\jmath)}(t), \alpha_{\Theta_{\imath}}^{(\jmath)}(t), \beta_{\Theta_{\imath}}^{(\jmath)}(t), \alpha_{\gamma_{2}}^{(\jmath)}(t), \beta_{\gamma_{\imath}}^{(\jmath)}(t)$ for $j=1,2$ (case 1 and case 2) are updated at each iteration $t$ according to (3.8), (3.12) and (3.13), respectively.

Also, note that in the proposed trust establishment scheme, we are using the existing information in the relay selection process. Therefore, our scheme does not impose communication overhead to the system for monitoring and measurements. It is worth mentioning that the proposed method is independent of the relay selection policy and the similar results can be drawn for other relay selection policies with independent decisions in different transmission frames.

It is worth mentioning that, if stochastic models assumed in the thesis are changed in practice the proposed method is still capable of estimating the trust values correctly. However, we can no more claim that the trusts follow the distribution. They will actually, follow a distribution which can derived by Bayes' rule in the Bayesian framework discussed in Chapter 2. The mean of the trust value distribution will converge to the proposed trust value in a different manner and speed.

The way by which the trust values affect the performance of the system depends on the network architecture and routing strategies. For instance, the derived trust values can be further used in the relay selection policy or in an Ad Hoc network, may be used for distinguishing the reliable nodes. A threshold can be set up to reject a node with the trust value lower than the threshold or nodes can still use the relays with low trust values but more resources may be assigned to the node with higher trust values to assure the higher throughput. This is the case where we propose a relay selection policy in the next chapter which is also throughput optimal. 


\subsection{Simulation Results and Discussions}

In this section, we will present the simulation results to show the effectiveness of the proposed trust establishment method. The topology used in the first part of simulations is the same as Figure 3.1 with 4 relay nodes to be verified for case 1 and case 2 as mentioned earlier. The system is using BPSK modulation without any coding scheme and the channels are slow Rayleigh fading channels. We assume that during each packet transmission frame, the fading coefficients are fixed. Since the variations of a channel are modelled by Rayleigh random variables the SNR will follow an exponential distribution. Thus, in the simulations we model the links SNRs by exponential random variables. We have assumed that the acceptable BER is $10^{-6}$ requiring the minimum SNR of $54 \mathrm{~dB}$ to achieve this BER for BPSK. Assume that the relays are malicious with probabilities $0.1,0.2,0.3$ and 0.4 , i.e., $E\left[Q_{1}\right]=0.1, E\left[Q_{2}\right]=$ $0.2, E\left[Q_{3}\right]=0.3$ and $E\left[Q_{4}\right]=0.4$. We have considered two situations of high and low SNR regimes for case 1 and case 2 .

In the high and low SNR regimes, the SNRs of the links $S-r_{\imath}$ and links $r_{\imath}-$ $D, \imath=1,2,3,4$ are exponential random variables with the mean equal to elements of vectors $(60,70,63,65) d B$ and $(52,49,54,50) d B$, respectively. In case 2 , the mean of exponential random variable of SNR for link $S-D$ is $50 d B$. The iteration length is assumed to be 100 frames, i.e., we observe the system every 100 frames.

\subsubsection{Case 1: Cooperative Relaying without Combining}

In Figures 3.3 and 3.4, we compare the trust values of the case 1 obtained from the proposed method and the conventional Bayesian trust establishment method. We expect to have trust values of $0.9,0.8,0.7$ and 0.6 for relay nodes 1 to 4 , respectively. In figures, only the trusts for relay nodes 1 and 3 are illustrated for clarity of figures. As shown, in both cases the proposed trust establishment scheme estimates the trust values of relay nodes 1 and 3 accurately. In contrast, the conventional Bayesian method is biased by channel conditions and the relay selection policy and cannot establish a reliable trust value for the relay nodes. In both figures, we observe that the conventional Bayesian method gives not only incorrect trust values for relay nodes 1 and 3 , but also it computes the trust of relay 1 below that of relay 3 . The reason is that in both cases, relay node 3 has better average channel condition rather than relay 1 . Therefore, it is more likely to select relay 3 for cooperation and packet 
forwarding rather than relay 1. It also has more probability of successful packet forwarding than relay 1 . Since the conventional Bayesian method does not consider the channel condition and relay selection policy in its trust computation process, it fails to calculate accurate and reliable trust values for the relays.

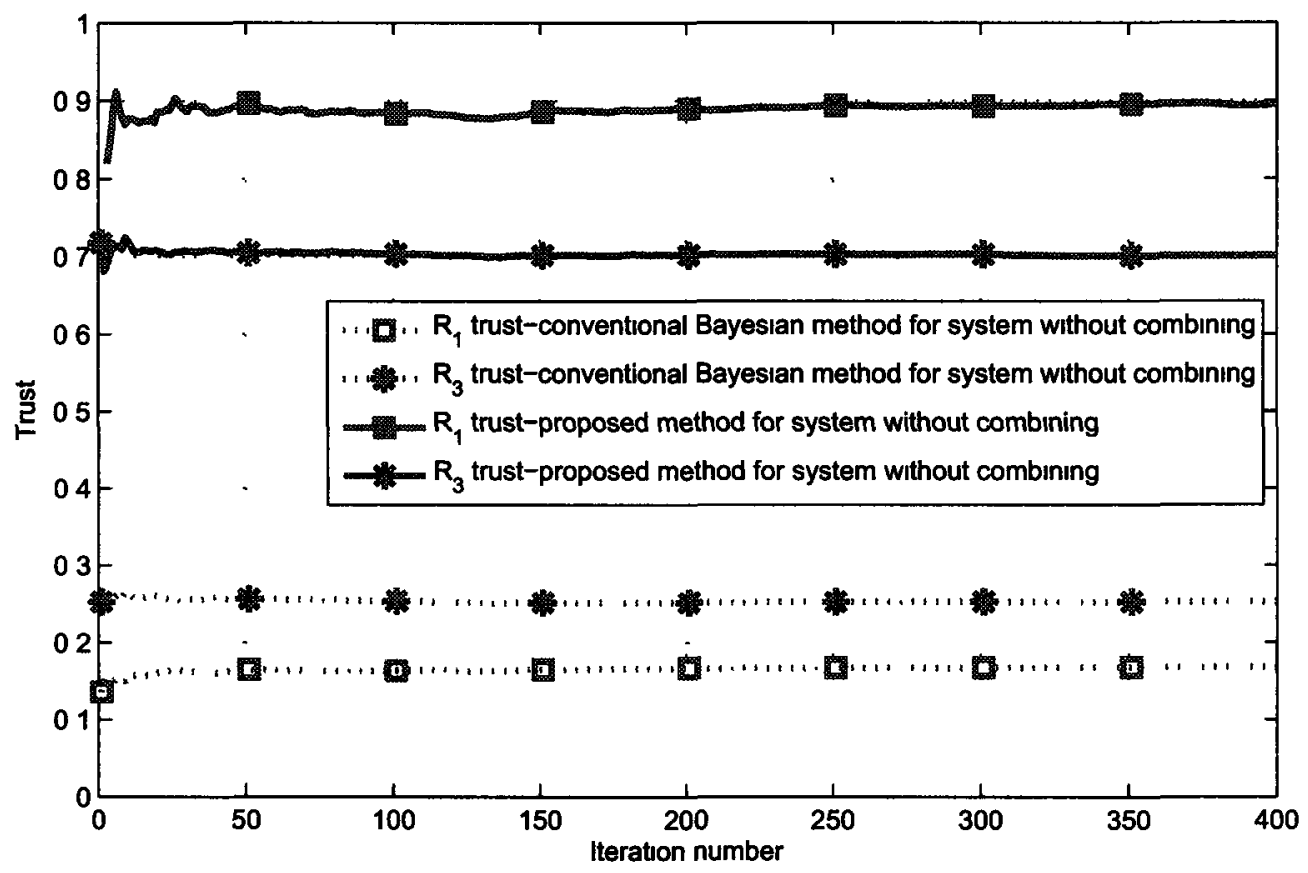

Figure 3.3: Trust establishment in the low SNR regime in case 1.

We can see the calculated trust of all relays at $t=300$ for both methods in high and low SNR regimes of the system without combining in Table 3.1 which admits the figure results.

Another point about these two figures is that in low SNR case (Figure 3.3), the conventional Bayesian method seems to give better estimates of trust rather than it does in the high SNR case (Figure 3.4). The reason is that in low SNR case, in contrast with high the SNR case, the average SNR of relays 1 and 3 are larger than relay nodes 2 and 4 . Therefore, the probability of selecting relay nodes 1 and 3 are higher than relay nodes 2 and 4 in the low SNR case. Thus, relay nodes 1 and 3 have more opportunity for forwarding the received packets in low SNR case rather than 


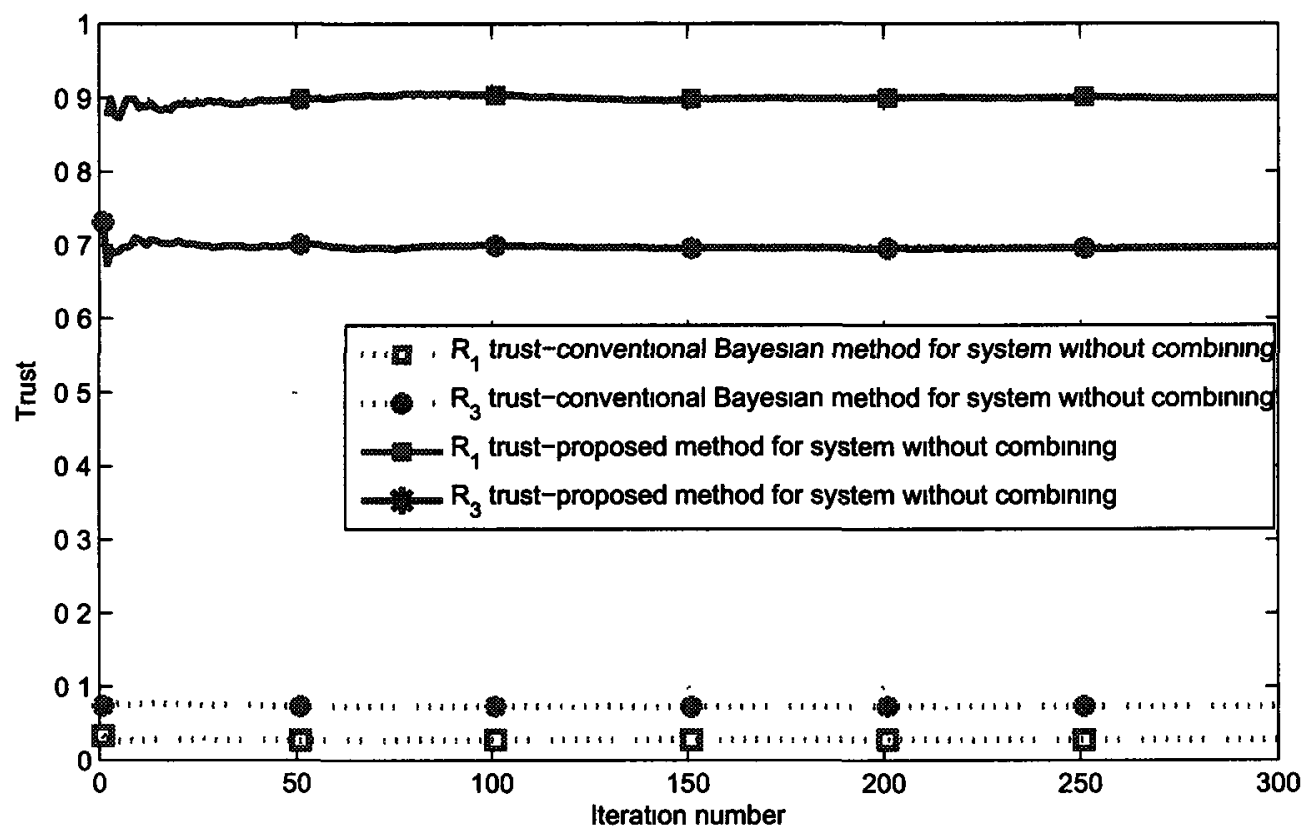

Figure 3.4: Trust establishment in the high SNR regime in case 1.

\begin{tabular}{|l|l|l|l|l|}
\hline \multirow{2}{*}{ Relay $i$} & \multicolumn{3}{|c|}{ Low SNR } & \multicolumn{2}{c|}{ High SNR } \\
\cline { 2 - 5 } trust $t=300$ & \multicolumn{3}{|c|}{ Scheme } \\
\cline { 2 - 5 } & Proposed & $\begin{array}{c}\text { Conventional } \\
\text { scheme }\end{array}$ & $\begin{array}{c}\text { Proposed } \\
\text { scheme }\end{array}$ & $\begin{array}{c}\text { Conventional } \\
\text { Bayesian }\end{array}$ \\
\hline \hline$T_{1}(300)$ & 0.900 & 0.500 & 0.903 & 0.027 \\
$T_{2}(300)$ & 0.801 & 0.722 & 0.800 & 0.569 \\
$T_{3}(300)$ & 0.698 & 0.538 & 0.697 & 0.073 \\
$T_{4}(300)$ & 0.596 & 0.452 & 0.598 & 0.123 \\
\hline
\end{tabular}

Table 3.1: Relay trusts in system without combining (case 1) 
high SNR case. This is another example of how channel conditions can skew results and expectations.

In another experiment, we simulated the case in which the malicious probability of a relay node changes at some point of time. Starting from the same initial conditions as above, we assume that at the 150-th iteration, maliciousness of relay node 2 changes to $E\left[Q_{2}\right]=0.5$. Note that the channel conditions are in the high SNR regime.

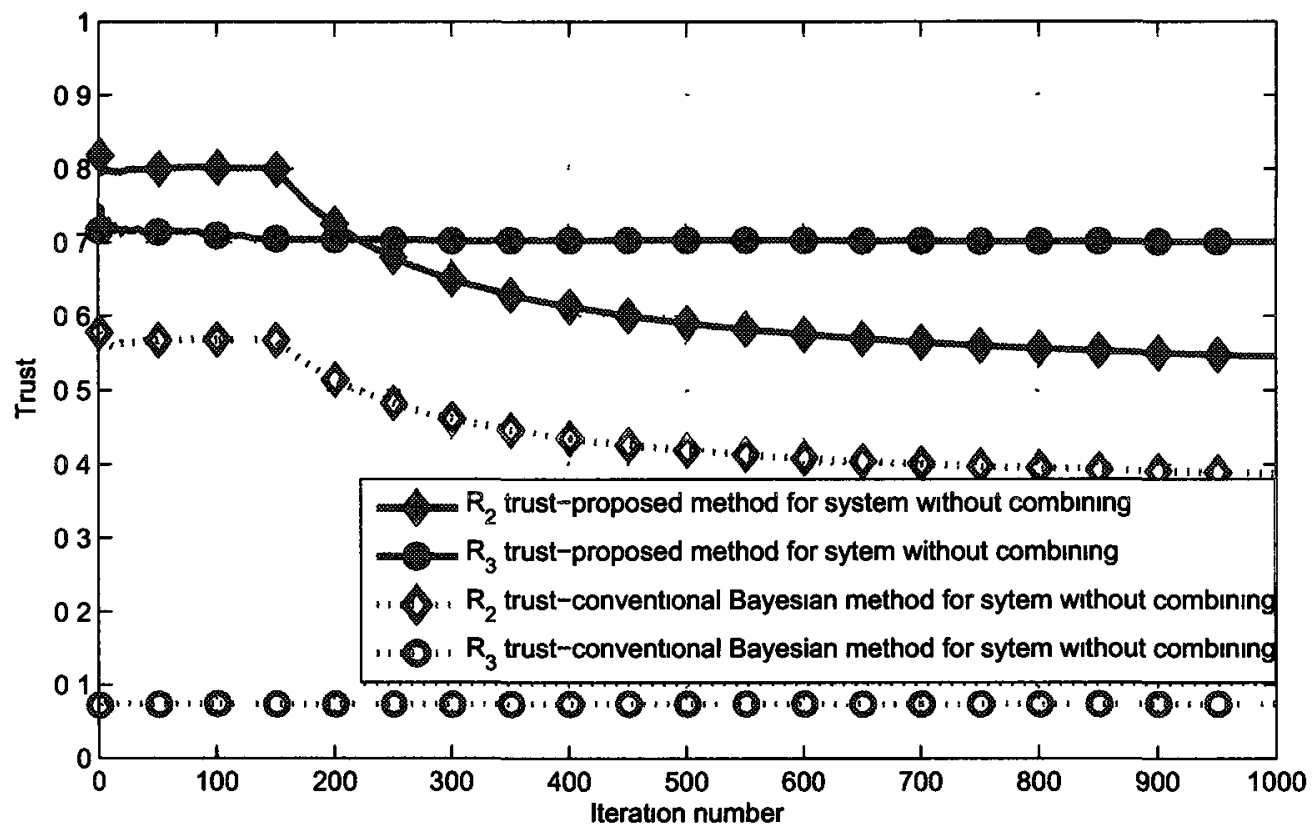

Figure 3.5: Trust tracking with time in case 1 .

In this experiment, we expect the value of relay node 3 to be 0.7 and relay 2 trust value should change from 0.8 to 0.5 after iteration 150 . This means that we expect to see the trust value of relay 2 falling below that of relay 3 after iteration 150 . As we can observe from Figure 3.5, the conventional Bayesian method not only gives incorrect trust estimations before and after iteration 150 , but it also does not satisfy our expectation about the inversion of the rankings of trusts after iteration 150 .

In contrast, Figure 3.5 shows that the proposed new method can track the trust through the time accurately. From Figure 3.5, we can also see that the response of the trust tracking is such that it cannot track rapid changes of trust in the system. To improve the speed of the tracking, we may further introduce a sliding window for the observation process as follows. In the trust computation of trust value at each 
iteration, we consider only the observations obtained inside a sliding window and discard the observations of the frames before the beginning of the sliding window. With a sliding window size of $\omega$, at iteration $t$, equation (3.8) becomes

$$
\begin{gathered}
\alpha_{\imath}(t)=\alpha_{\imath}(t-1)+\ell_{\imath}(t)-\ell_{\imath}(t-\omega), \\
\beta_{\imath}(t)=\beta_{\imath}(t-1)+k_{\imath}(t)-\ell_{\imath}(t)-k_{\imath}(t-\omega)+\ell_{\imath}(t-\omega), \\
\alpha_{\imath}(0)=1, \quad \beta_{\imath}(0)=1, \quad k_{\imath}(\tau)=0, \quad \ell_{\imath}(\tau=0), \quad \forall \tau \leq 0 .
\end{gathered}
$$

Using equations (3.14) and (3.15) the trust at each iteration $t$ is calculated for the window based approach. Figure 3.6 compares the trust for the proposed scheme with and without applying the sliding window. In our simulation, we set $\omega$ equals to 50 iterations. As we can observe from Figure 3.6, trust tracking is much more faster in the case where a sliding window is used.

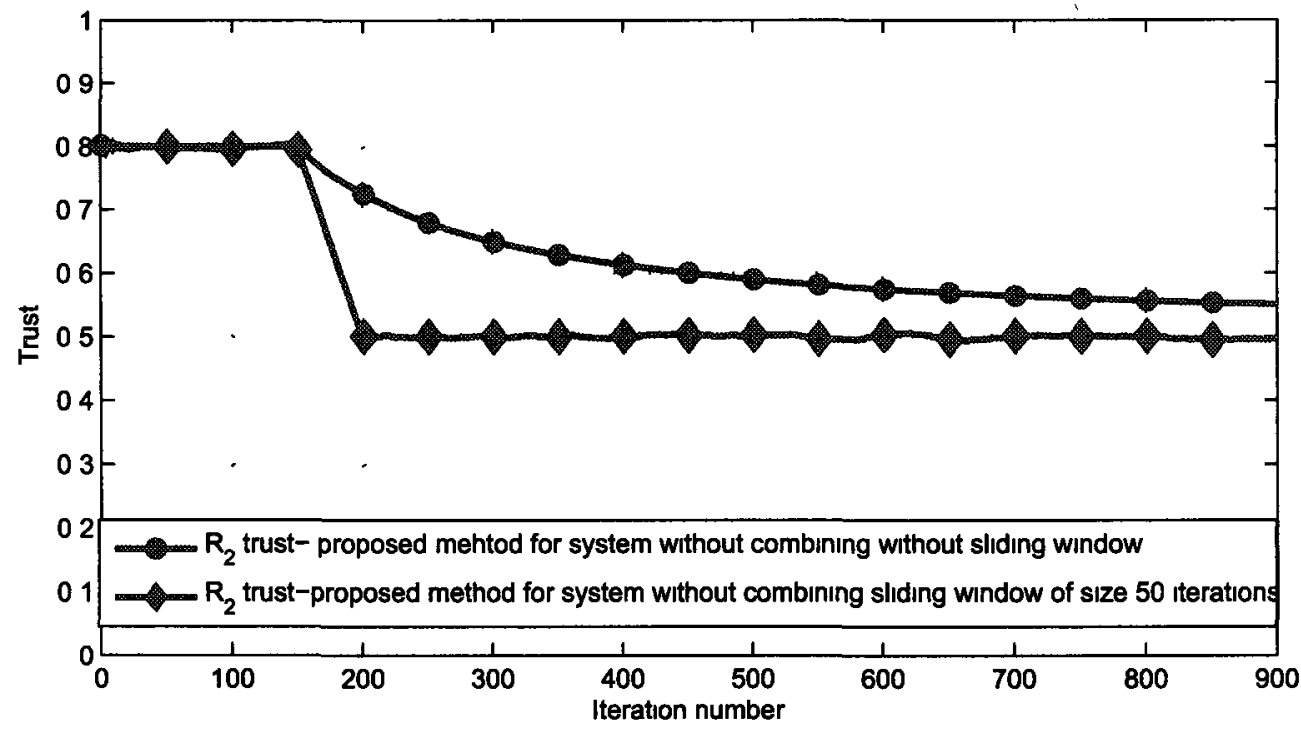

Figure 3.6: Trust tracking with and without using a sliding window in case 1.

Note that selection of an appropriate window size is very important in this case. That is selection of a large window size may result in delay in trust change tracking (like Figure 5 as the extreme case) and selection of a small window size may result in oscillations of the computed trust. 


\subsubsection{Case 2: Cooperative Relaying with Combining}

In Figures 3.7 and 3.8, we compare the trust values of the case 2 obtained from the proposed method and the conventional Bayesian trust establishment method in high and low SNR regimes. We expect to have trust values of $0.9,0.8,0.7$ and 0.6 for relay nodes 1 to 4 , respectively. Only the trusts for two relay nodes are illustrated due to space limitations. As shown, in both SNR regimes the proposed trust establishment scheme estimates the trusts of two nodes accurately. In contrast, similar to case 1 , the conventional Bayesian method is biased by channel conditions and the relay selection policy and cannot establish an accurate trust value for the relay nodes. In Figure 3.7 and Figure 3.8, we observe that the conventional Bayesian method gives incorrect trust values for relay nodes 1 and 3 .

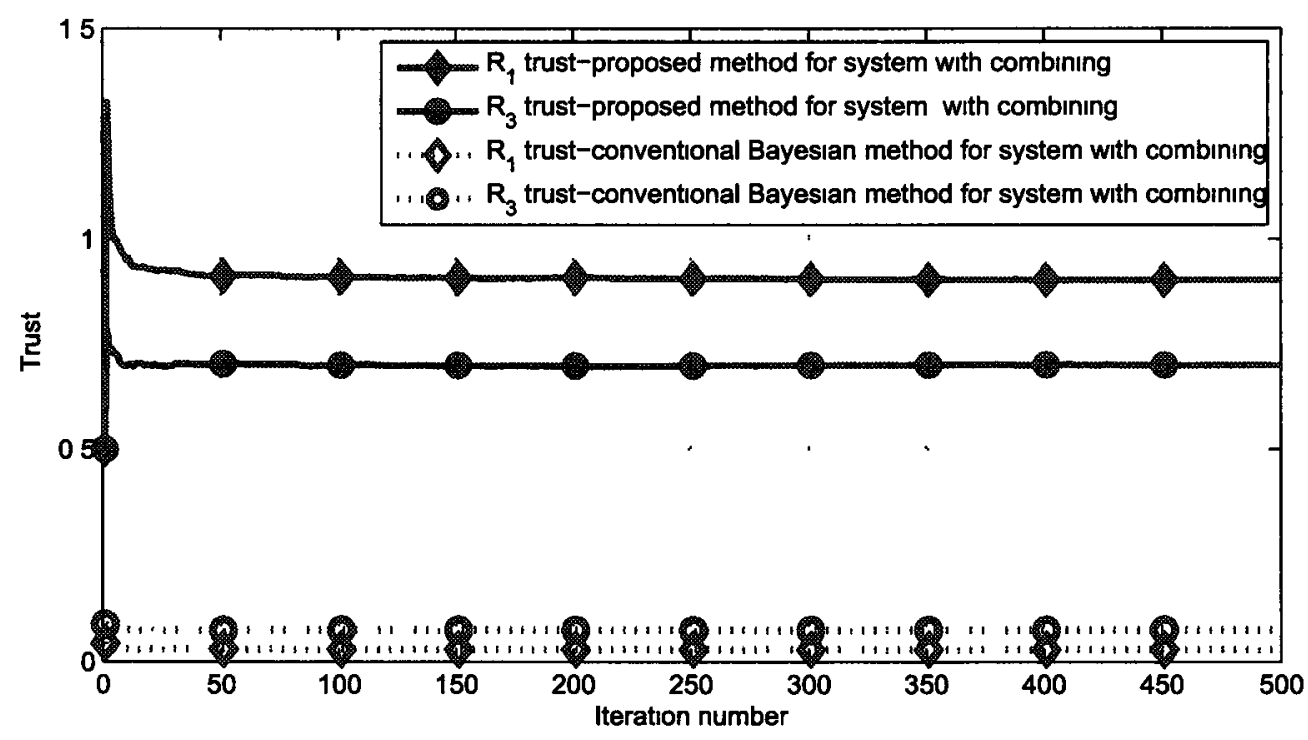

Figure 3.7: Trust establishment in high SNR regime in case 2 .

In Table 3.2, we can easily observe the trust of all relays at $t=300$ both for proposed method and conventional method in high and low SNR regimes in the system with combining at destination, i.e., case 2 . As we expect the results in the table admit the figures results.

In another experiment, similar to an experiment in section 3.4.1, we simulated the 


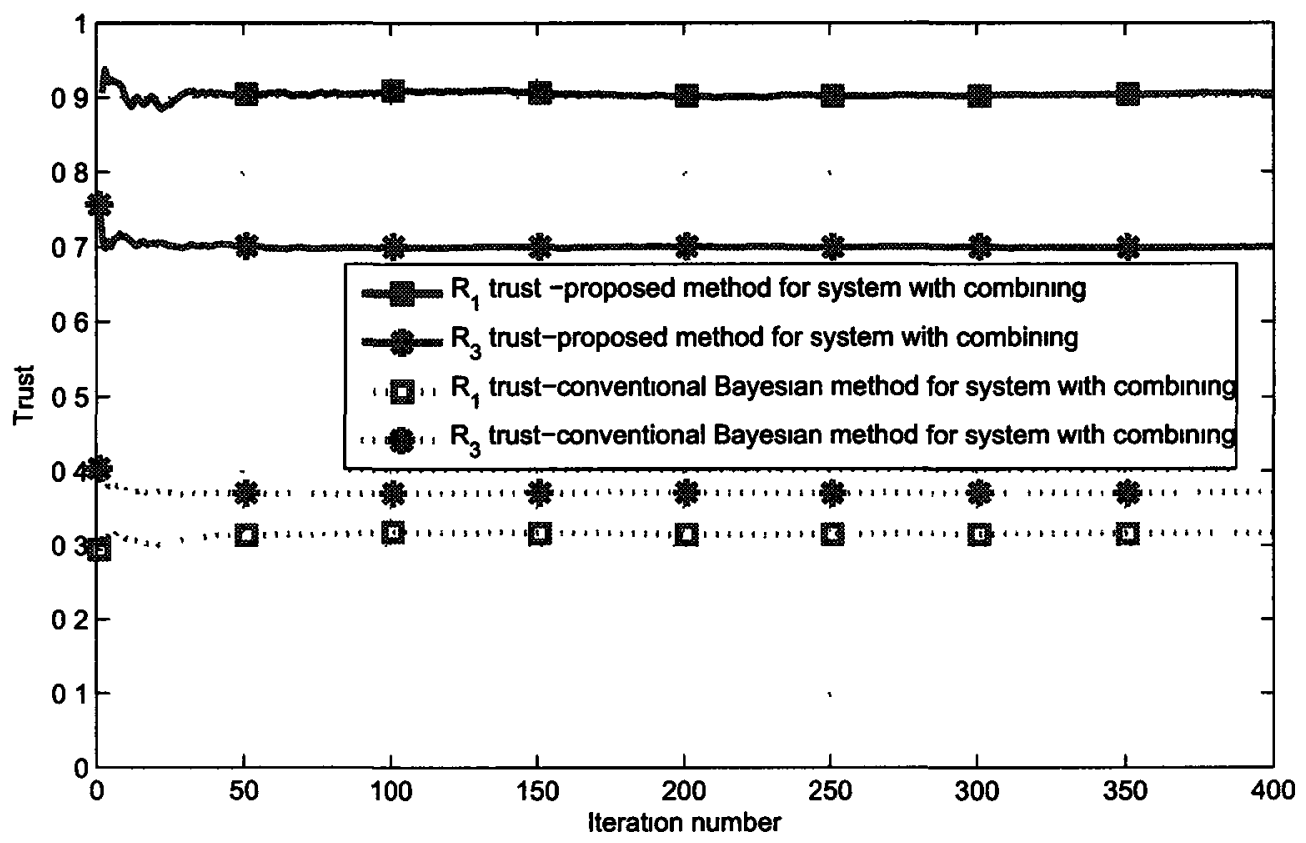

Figure 3.8: Trust establishment in low SNR regime in case 2.

\begin{tabular}{|c|c|c|c|c|}
\hline \multirow[b]{3}{*}{$\begin{array}{c}\text { Relay } i \\
\text { trust } t=300\end{array}$} & \multicolumn{2}{|c|}{ Low SNR } & \multicolumn{2}{|c|}{ High SNR } \\
\hline & \multicolumn{4}{|c|}{ Scheme } \\
\hline & $\begin{array}{c}\text { Proposed } \\
\text { scheme }\end{array}$ & $\begin{array}{c}\text { Conventional } \\
\text { Bayesian }\end{array}$ & $\begin{array}{c}\text { Proposed } \\
\text { scheme }\end{array}$ & $\begin{array}{c}\text { Conventional } \\
\text { Bayesian }\end{array}$ \\
\hline$T_{1}(300)$ & 0.895 & 0.027 & 0.902 & 0.028 \\
\hline$T_{2}(300)$ & 0.799 & 0.568 & 0.800 & 0.568 \\
\hline$T_{3}(300)$ & 0.700 & 0.073 & 0.703 & 0.074 \\
\hline$T_{4}(300)$ & 0.600 & 0.123 & 0.602 & 0.124 \\
\hline
\end{tabular}

Table 3.2: Relay trusts in system with combining (case 2) 
situation where the maliciousness probability of a relay node changes at some point of the time. Again, starting from the same initial conditions as above, we assume that at the 150-th iteration, maliciousness of relay node 2 changes to $E\left[Q_{2}\right]=0.5$. The channel conditions are in the high SNR regime.

In this experiment, we expect the trust value of relay node 4 to be 0.6 and trust of relay 2 should change from 0.8 to 0.5 after iteration 150 . That is, we expect to see the trust value of relay 2 falling below that of relay 3 after iteration 150 . As we can easily observe in Figure 3.9, the conventional Bayesian method gives incorrect trust estimations before and after iteration 150 . It also does not satisfy our expectation about the inversion of the rankings of trusts after iteration 150 .

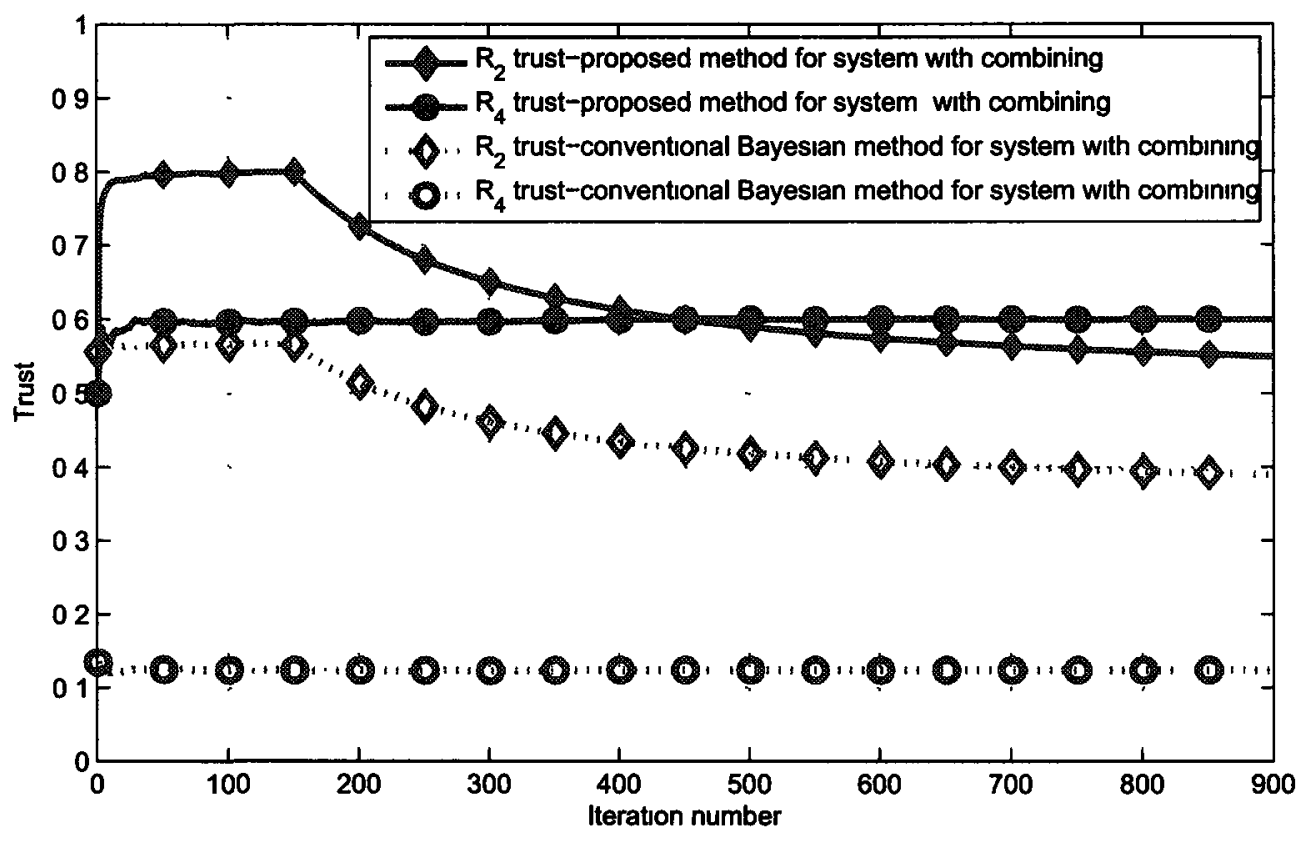

Figure 3.9: Trust tracking with time in system in case2.

In contrast, Figure 3.9 shows that the proposed new method can track the trust through the time accurately. As we see in Figure 3.9, the response of the trust tracking is not such that it can track rapid changes of trust in the system.

To improve the speed of the tracking, again we may use a sliding window for the observation process as described earlier in section 3.4.1. With a sliding window size of $\omega$, at iteration $t$ and using equations (3.14) and (3.15) the trust at each iteration $t$ 


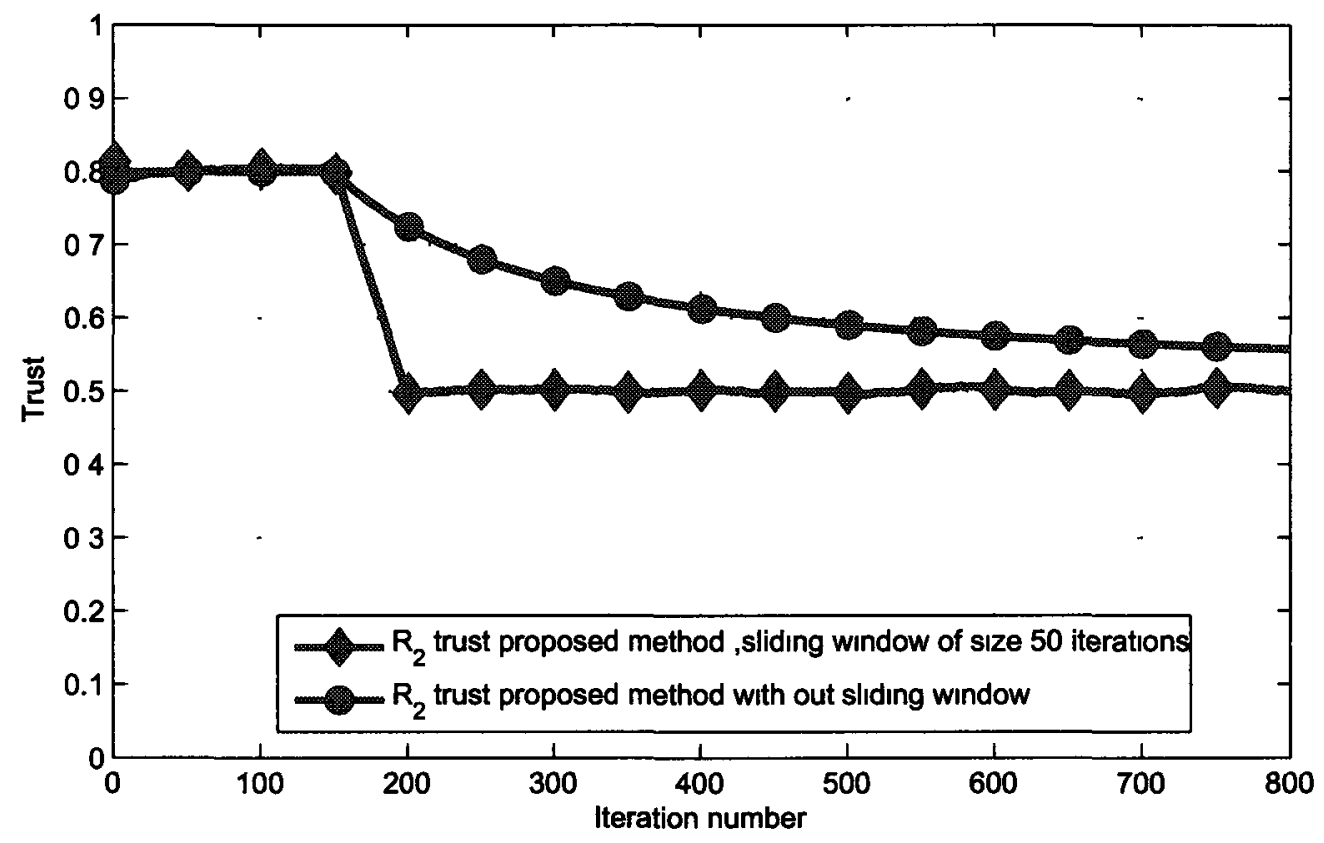

Figure 3.10: Trust tracking with and without using a sliding window in case 2 .

is calculated for the window based approach. Figure 3.10 compares the trust for the proposed scheme with and without applying the sliding window where $\omega$ equals to 50 iterations. Similar to case 1 , we observe that trust tracking is much more faster in the case where a sliding window is used.

In another experiment, the topology shown in Figure 3.11 was used to observe how the proposed trust establishment works in the presence of malicious relays in the multi-user cooperative networks for a different policy. What makes this experiment distinct from the previous one is that there are 4 users and 3 relays for the cooperation. Each user has a specific channel for its communication. Relays are capable of tuning to the users channel and detecting the users data simultaneously. In the first slot of a transmission frame, users send their data to all the relays. In the second time slot of every transmission frame, each relay must be assigned to a user based on the following policy. Those users whose data is received properly at a relay are candidates for assignment to that specific relay. Hungarian algorithm which is a solution to maximum matching problem was applied as the policy of relay assignment to the users so that the maximum connectivity in the system can be achieved [81] . All other physical layer characteristics of the simulated system is the same as before. 
Each relay may have a different probability of maliciousness with respect to different users. Maliciousness parameters of relays of the multi-user system which we simulated are indicated by a matrix $q$ as follows.

$$
q=\left(\begin{array}{ccc}
0.8 & 0 & 0.8 \\
0.6 & 0 & 0.6 \\
0.4 & 0 & 0.4 \\
0.2 & 0 & 0.2
\end{array}\right)
$$

Each element of matrix $q, q_{\jmath, 2}$ where $n \in\{1,2,3,4\}, i \in\{1,2,3\}$ is in fact $E\left[Q_{\jmath, 2}\right]$ and denotes probability of maliciousness of relay $i$ with respect to user $j$. Equivalently, $T_{n, 2}$ is trust of relay $i$ with respect to user $n$.

In Figure 3.11, for clarity of the figure, only 4 of these trust values are depicted. $T_{1,1}$, the trust value of relay 1 with respect to user 1 , i.e., $1-E\left[Q_{1,1}\right]$ is expected to be 0.2 . Similarly, we expect $T_{2,3}$, trust value of relay 3 for user 1 to be $0.4, T_{3,1}$ to be 0.6 and $T_{4,3}$ to be 0.8 , respectively. As it is shown in Figure 3.12, the proposed trust establishment can correctly calculate the trust value of a relay with respect to a specific user for such relay assignment policy in the situation that a malicious relay behaves differently and discriminates among users.

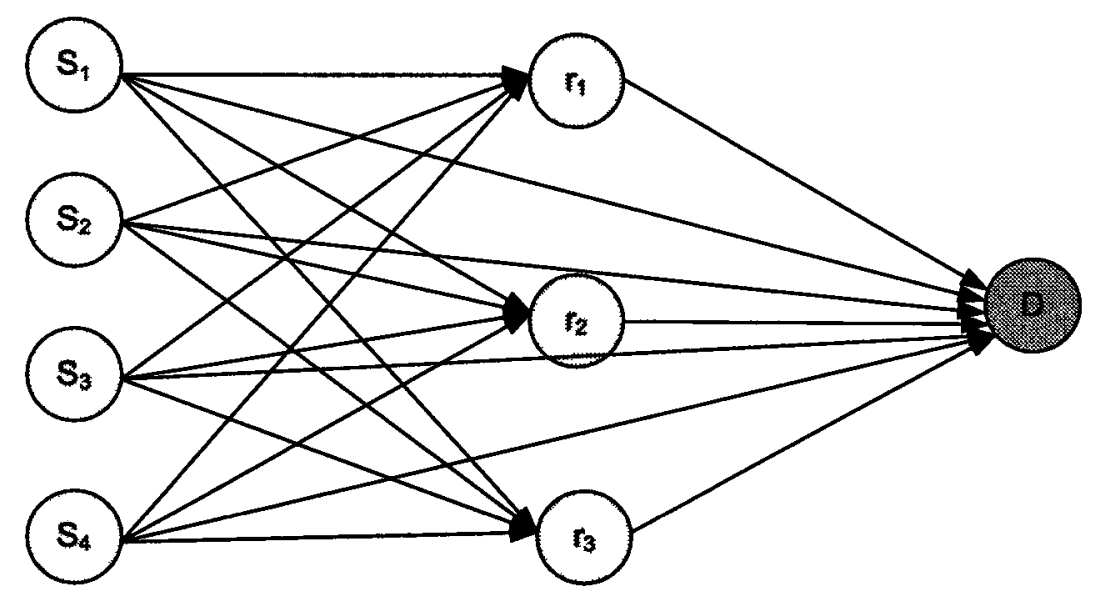

Figure 3.11: Relays trust of multi-user system in high SNR regimes 


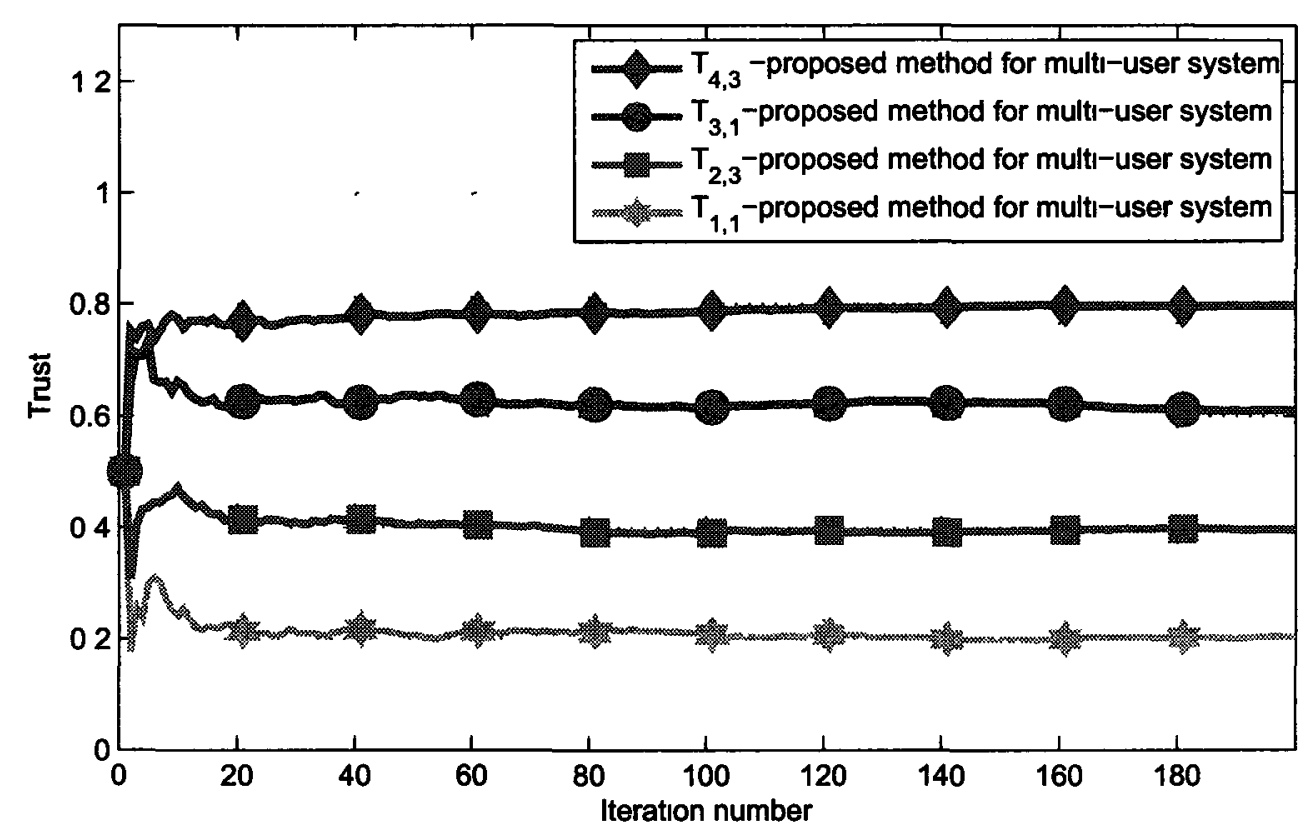

Figure 3.12: Relays trust of multi-user system in high SNR regimes

\subsection{Summary}

In this chapter, we introduced a trust establishment scheme for cooperative wireless networks. The proposed scheme covers a broad range of models in which the channel conditions and the relay selection decisions are independent in different transmission frames. We showed that the conventional a Bayesian trust establishment method is insufficient for this task as it is biased by the channel conditions and relay selection decision processes. We modified the conventional trust establishment method by incorporating the available information about the channel conditions and relay selection decisions. By using simulations we demonstrated the effectiveness of the proposed method. In the proposed method we always assume that the transmissions, relay selections or channel conditions are independent across different frames. In the simulations, we also observed that the calculated trust values may lag the true values in cases of trust change in the system. Therefore, we introduced a sliding window in the system to compute trust at each iteration. Simulation results confirm the improvement in the speed of trust change tracking. 


\section{Chapter 4}

\section{Optimal Secure Relay Selection in Multiuser Cooperative Relaying Networks}

\subsection{Introduction}

The problem of optimal secure relay selection in multiuser cooperative wireless networks is investigated in this chapter. A general discrete time model for such networks is introduced which takes into account the dynamic variations of the channel state, the stochastic malicious behaviour of relay nodes as well as stochastic arrival of data packets into the system. The model consists of a set of mobile users, one destination node and a set of relay nodes which may be either mobile or fixed. The system uses the benefit of cooperative diversity by relaying in decode and forward mode. We assume that each user either transmits its packets directly to the destination or selects a relay node to cooperatively transmit its packets. A malicious relay node may behave maliciously and refuse to cooperative with a user deliberately when it is selected for cooperation. A malicious relay node usually does this stochastically to hide his malicious behaviour for longer time. Therefore, at each time slot we have to decide whether a user has to cooperate with any relay node or not and if so, which relay node should be selected for cooperation.

Note that in all the relay selection policies of earlier works mentioned in Chapter 2, the relay nodes are assumed to be trusted relay nodes. However, As we discussed earlier, security is a challenging issue in cooperative relaying networks where the source node relies on intermediate relay nodes to transmit its information. Although cryptographic techniques provide assuring approaches to make the system secure, there still remains the chance for compromised relay nodes to take part in the cooperation 
process and disrupt the transmissions. Another example is the case where a selfish (and not necessarily compromised) relay node refuses to cooperate or deliberately drops the received packets (for different reasons e.g. power saving) when it is selected for cooperation. Henceforth, we will refer to such misbehaving relay nodes as malicious nodes. To quantify the maliciousness of relay nodes we will use the trust values associated to the relay nodes. As we discussed earlier, trust values are real values from the interval $[0,1]$ which are a measure of trustworthiness of relay nodes.

We will propose a secure throughput optimal relay selection policy that can stabilize the system for all the arrival rate vectors strictly inside the stability region. Then, we show that the optimal policy is equivalent to finding the maximum matching in a weighted bipartite graph at each time slot. We also use simulations to compare the performance of the proposed policy with four other sub-optimal policies in terms of average queue occupancy (or equivalently, queueing delay) and stable throughput region. In the proposed policy we incorporate the trust values associated to each relay node, queue length process and the channel state of the system to decide about relay assignment at each time slot. Note that the trust values employed in the proposed policy are general trust values which are real numbers from interval $[0,1]$ which reflect how much reliable is the relay node in cooperation with a specific user. The trust values may be the output of any intrusion detection or anomaly detection tool or trust establishment method.

The results in this chapter are in fact an extension of the work in [48] where all the relay nodes were assumed to be trusted. The main goal of this chapter is to devise an optimal secure relay selection policy in a multiuser cooperative wireless system. In our model, there is a set of users as well as a set of relay nodes and a centralized controller that controls the relay assignment process. Each user may transmit directly to the destination (direct mode) or it can send its packets to the destination using the cooperation with a relay node according to a two phase cooperative protocol (cooperation mode). Therefore, at each time slot, the network controller has to address two questions about each user: whether it should work in the direct mode or cooperation mode; and if it has to cooperate, which relay node must be assigned to that user. We call any strategy that answers these two questions about each user a relay selection policy. We investigate the optimal policy for such a problem with the objective of maximizing the long run average throughput of the system. The optimal policy does its duty based on the available information about the channel state of 
the system, trust values corresponding to each relay node as well as the length of each user's queue. We will characterize the optimal policy as an integer programming (IP) problem. Then, we will show that the IP problem at each time slot is equivalent to finding the maximum matching in a weighted bipartite graph. Simulations are conducted to compare the performance of the proposed policy with other cooperative and non-cooperative policies.

The rest of this chapter is organized as follows. In section 4.1.1, we will describe the model used in this chapter and then we will have a brief review on the notions of stability region and throughput optimal policies. In section 4.2 , we will introduce the secure throughput optimal relay selection policy. We also show that the optimal policy is equivalent to finding the maximum weighted matching in a bipartite graph at each time slot. Simulation results are brought in section 4.3. Section 4.4 summarizes the conclusions.

\subsubsection{Problem Formulation}

\section{Model Description}

The cooperative relaying model which is used in this chapter is similar to the model used in [48]. It consists of the set of users $\mathcal{N}$ with $|\mathcal{N}|=N$, the set of relay nodes $\mathcal{R}$ with $|\mathcal{R}|=R$ and one destination node $D$. Although the system is assumed to be working in uplink, the same results can be easily concluded for downlink as well. Suppose that there is a separate channel $C_{n}$ assigned to each user $n$ whose transceiver is tuned on that channel. Therefore, the destination node $D$ is able to receive and decode the information transmitted from all users, simultaneously. The system is assumed to be time slotted with equal length intervals.

The exogenous packet arrival process to each user $n$ at each time slot $t$ is denoted by $A_{n}(t)$. In fact, $A_{n}(t)$ represents the number of packets arrived for user $n$ exogenously during time slot $t$. It is assumed that $E\left[A_{n}^{2}(t)\right]<\infty$ for all $t$ and all $n$. Note that in our model the new arrivals are added to each queue at the end of each time slot. The arrival processes $A_{n}(t) \forall n \in \mathcal{N}$ are assumed to be i.i.d. with mean $\lambda_{n}$. Suppose that $U(t)=\left(U_{1}(t), \ldots, U_{N}(t)\right)$ is the queue length process vector at the end of time slot $t$ after adding new arrivals to the queues. Figure 4.1 illustrates the model used in this chapter.

At each time slot, packet transmissions from each user $n$ to $D$ may be performed 


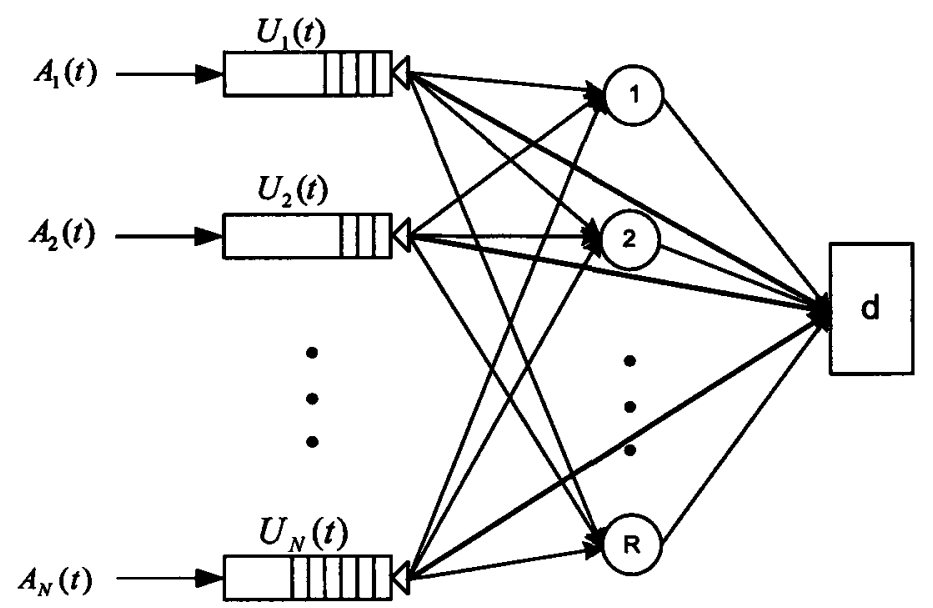

Figure 4.1: The model of cooperative communication used in this chapter

in direct mode or cooperative mode. In the former case, user $n$ transmits its packets directly to $D$ and in the latter case it uses one of the relay nodes to transmit its packets cooperatively.

A user $n$ in direct mode uses all the time slot duration to transmit its packets. However in cooperative mode, it uses a two phase transmission protocol to transmit its packets to $D$ (relay nodes are assumed to be half duplex). In this case, the time slot duration is split into two sub-slots. In the first sub-slot, the user $n$ starts the first phase of transmission in which it broadcasts its packets to the relay nodes as well as $D$. In the second sub-slot, a relay node $r$ is selected for cooperation with user $n$ according to a relay selection policy and then it cooperatively relays the received packets from user $n$ to the destination node $D$.

The relay nodes are assumed to work in Decode and Forward (DF) mode in which each relay node $r$ receives the signal transmitted in the first phase and decodes it. Then, it re-encodes the information and forwards it to $D$. Note that the results obtained in this chapter are also true for systems which support Amplify and Forward (AF) relaying mode. However, in our model, all the relays are in DF mode. In AF mode, the relay node $r$ receives the signal but does not decode it. However, it amplifies the signal in such a way that its transmission power satisfies the relay's maximum power constraint. In both cases of $\mathrm{DF}$ and $\mathrm{AF}$, destination node $D$ uses the information received from direct link in the first sub-slot and the relayed information in the second sub-slot and combines them. Then, $D$ uses the combined signal to 
decode the information.

Suppose that the direct communication link between each user $n, n \in \mathcal{N}$ and node $D$ is denoted by $\ell_{n, D}$. Let $R_{n, D}(t)$ represent the achievable rate corresponding to each link $\ell_{n, D}$ (which is using channel $C_{n}$ ) at time slot $t$. The achievable rates over different communication links are varying from time slot to time slot. The variation of communication links is due to various factors such as fading, mobility of the users, path loss, etc. Assume that $H_{n, D}(t)$ denote all the effects of such factors on each link $\ell_{n, D}$ during time slot $t$. Henceforth, we call $H_{n, D}(t)$ the channel gain of direct link $\ell_{n, D}$ at time slot $t$. Therefore, if $X_{n, D}(t)$ and $Y_{n, D}(t)$ denote the symbol transmitted and received on this link, respectively, then $\forall n \in \mathcal{N}$

$$
Y_{n, D}(t)=H_{n, D}(t) X_{n, D}(t)+Z_{D}(t)
$$

$Z_{D}(t)$ is the additive Gaussian noise process at node $D$ with Power Spectral Density (PSD) of $\frac{N_{0}}{2}$. According to Shannon formula for capacity of communication channels, the rate $R_{n, D}(t)$ is determined by

$$
R_{n, D}(t)=W_{n} \log _{2}\left(1+\frac{\left|H_{n, D}(t)\right|^{2} P_{n}}{N_{0} W_{n}}\right) \text { bits } / \mathrm{sec}
$$

where $P_{n}$ is the transmission power of user $n$ and $W_{n}$ is the bandwidth of the channel $C_{n}$. Note that $R_{n, D}(t)$ is the maximum achievable transmission rate for reliable communication over each link $\ell_{n, D}$ at time slot $t$.

The cooperative link consisting of user $n$, relay node $r$ and destination node $D$ is represented by $\ell_{n, r}$. The achievable rate corresponding to the cooperative link $\ell_{n, r}$ (which is in DF mode) at time slot $t$ is denoted by $R_{n, r}^{D F}(t)$. This rate depends on the channel gains of direct links between $n$ and $r$, the gain of direct link between $n$ and $D$ and the gain of direct link between $r$ and $D$ denoted by $H_{n, r}(t), H_{n, D}(t)$ and $H_{r, D}(t)$, respectively. If each relay node $r$ has the maximum power limit of $P_{r}^{\prime}$ for its transmissions, the achievable rate $R_{n, r}^{D F}(t)$ is calculated by the following equation [8].

$$
\begin{aligned}
R_{n, r}^{D F}(t)= & \frac{W_{n}}{2} \min \left\{\log _{2}\left(1+\frac{\left|H_{n, r}(t)\right|^{2} P_{n}}{N_{0} W_{n}}\right),\right. \\
& \left.\log _{2}\left(1+\frac{\left|H_{n, D}(t)\right|^{2} P_{n}}{N_{0} W_{n}}+\frac{\left|H_{r, D}(t)\right|^{2} P_{r}^{\prime}}{N_{0} W_{n}}\right)\right\}
\end{aligned}
$$

Note that the achievable rate for DF in (4.2) is for the case where a repetition 
code is used at the relay node, i.e., the relay node is using the same codebook as the source is using $[8,31]$.

It is worth mentioning that if relay nodes operate in AF relaying mode, then the achievable rate corresponding to the cooperative link $\ell_{n, r}$ is denoted by $R_{n, r}^{A F}(t)$ and is calculated by the following equation [8].

$$
\begin{aligned}
R_{n, r}^{A F}(t)=\frac{W_{n}}{2} & \log _{2}\left(1+\frac{\left|H_{n, D}(t)\right|^{2} P_{n}}{N_{0} W_{n}}\right. \\
& \left.+f\left(\frac{\left|H_{n, r}(t)\right|^{2} P_{n}}{N_{0} W_{n}}, \frac{\left|H_{r, D}(t)\right|^{2} P_{r}^{\prime}}{N_{0} W_{n}}\right)\right)
\end{aligned}
$$

where

$$
f(x, y):=\frac{x y}{x+y+1}
$$

Thus, by having the channel gains of all one hop communication links, we can easily determine the achievable rates of all the direct links $\left(R_{n, D}(t), n=1,2, \ldots, N\right)$ and cooperative links $\left(R_{n, r}^{D F}(t), n=1,2, \ldots, N, r=1,2, \ldots, R\right)$.

The channel gains are assumed to be fixed during each time slot. However, they are allowed to vary across different time slots. This is the usual assumption when modelling a slow Rayleigh fading wireless channel. The channel state of the system is defined as the set of channel gains of all one hop links in the systems, i.e.,

$$
C S I(t)=\left\{H_{n, D}(t), H_{n, r}(t), H_{r, D}(t), \forall n \in \mathcal{N}, \forall r \in \mathcal{R}\right\}
$$

It is assumed that the channel state $C S I(t)$ evolves with time according to a finite state, irreducible Markov chain. Therefore, the system has stationary sate probabilities and hence the capacity region of the system is well defined [9]. Although the channel state of the system has Markovian property, the channel gains over different links at any time slot $t$ may be arbitrarily correlated.

Packets are assumed to have the same length which is equal to $L$. Therefore, the number of packets that can be transmitted successfully through the direct link $\ell_{n, D}$ and the cooperative link $\ell_{n, r}^{D F}$ are equal to

$$
w_{n, D}(t)=\left\lfloor\frac{R_{n, D}(t)}{L}\right\rfloor, w_{n, r}(t)=\left\lfloor\frac{R_{n, r}^{D F}(t)}{L}\right\rfloor
$$


respectively.

The maliciousness of each relay node $r$ with respect to user $n$ is further modelled by a binary random variable $Q_{n, r}$ with parameter $q_{n, r}$. In other words, at each cooperative transmission over cooperative link $\ell_{n, r}$, a malicious relay node $r$ behaves maliciously and does not forward the packets received in the first phase with probability $q_{n, r}$. Stochastic modelling of the maliciousness of a node has appeared previously in the literature, e.g., $[5,72,80]$. In fact, a clever attacker may use stochastic malicious behaviour to hide his maliciousness behind the uncertainty of the wireless channel [80] and keep his malicious behaviour for longer time. Note that in our model a malicious relay node $r$ may have different malicious behaviour to different users. This is a general assumption in which malicious relays may attack some specific users.

We assume that a centralized controller at each time slot determines each user's mode of operation (direct or cooperation) and if it is in cooperation mode, which relay must be selected for cooperation such that the long-term throughput region of the system is maximised. This is accomplished based on the information about the channel state and also the queue length process $U(t)$.

Thus, a relay selection policy may be defined by determination of the following indicator variables $I_{n, D}(t)$ and $I_{n, r}(t)$ at each time slot $t$.

$$
\begin{gathered}
I_{n, D}(t)=\left\{\begin{array}{lc}
1 & \text { user } n \text { transmits directly to } D \text { at time } t \\
0 & \text { Otherwise }
\end{array}\right. \\
I_{n, r}(t)=\left\{\begin{array}{lc}
1 & \text { user } n \text { selects relay node } r \text { at time } t \text { for cooperation } \\
0 & \text { Otherwise }
\end{array}\right.
\end{gathered}
$$

Note that not all the allocations of $I_{n, D}(t)$ and $I_{n, r}(t)$ are valid. In fact, these indicator variables are constrained to satisfy the following conditions.

$$
\sum_{n=1}^{N} I_{n, r}(t) \leq 1 \quad \forall r=1,2, \ldots, R
$$




$$
I_{n, D}(t)+\sum_{r=1}^{R} I_{n, r}(t) \leq 1 \quad \forall n=1,2, \ldots, N
$$

The above constraints comes from the facts that each relay can be assigned to at most one user and each user may be in direct mode or cooperative mode and if it is in cooperative mode, it can cooperate with at most one relay node.

\section{Stability Region and Throughput Optimal Policies}

The concepts of stability, stability region and throughput optimal policies in stochastic network optimization comes from the works $[6,9,82,83]$. First, we will review the definition of strong stability in queueing networks [9]. A queuing system is defined as stable if in the long run, average queue sizes of the network nodes remain bounded and do not grow to infinity. Consider a discrete time single queue system with an arrival process $A(t)$ and service process $\mu(t)$. Suppose that $U(t)$ denotes the queue length process at time slot $t$. The evolution of $U(t)$ with time is determined by the following rule.

$$
U(t)=(U(t-1)-\mu(t))^{+}+A(t)
$$

where $(\cdot)^{+}$outputs the term inside the brackets if it is non-negative and zero otherwise.

Definition $1 A$ discrete time single queue with an arrvval process $A(t)$ and service process $\mu(t)$ is called strongly stable if it has bounded time averaged expected queue occupancy [9], i.e.,

$$
\limsup _{t \rightarrow \infty} \frac{1}{t} \sum_{\tau=0}^{t-1} E[U(\tau)]<\infty
$$

Definition 1 can be extended to a queueing network as follows [9].

Definition 2 A queueing system is called to be strongly stable if all the queues in the system are strongly stable. Specifically, for a network consisting of $N$ queues, the system is strongly stable if the time averaged expected aggregated backlog in the network is bounded, i.e.,

$$
\underset{t \rightarrow \infty}{\limsup } \frac{1}{t} \sum_{\tau=0}^{t-1} \sum_{n=1}^{N} E\left[U_{n}(\tau)\right]<\infty .
$$


Consider the queueing system in definition 2 with $N$ queues. Assume that the arrival rate to each queue $n$ is represented by $\lambda_{n}$ and therefore, the arrival rate vector is denoted by $\lambda=\left(\lambda_{1}, \lambda_{2}, \ldots, \lambda_{N}\right)$. A resource allocation (relay selection) policy $\pi$ is said to stabilize the system if by applying policy $\pi$ the system is strongly stable. Let $\Lambda_{\pi}$ represent the closure of the set of arrival rate vectors for which $\pi$ can stabilize the system. $\Lambda_{\pi}$ is called the stability region for policy $\pi$. Now, consider the union of the stability region of all the possible policies and let us denote it by $\Lambda$, i.e., $\Lambda=\bigcup_{\pi} \Lambda_{\pi}$. $\Lambda$ is called the network stability region (also called network layer capacity region in literature [9]). In other words, the network stability region is the closure of the set of all arrival rate vectors for which there exists a policy that can stabilize the network. Note that the network stability region is independent of the resource allocation policy and in fact is a specific characteristic of any network. A resource allocation policy $\pi^{*}$ is called throughput optimal if it can stabilize the system for all the arrival rate vectors strictly inside the network stability region. In other words, $\pi^{*}$ is throughput optimal if its stability region coincides with the network stability region.

Now, consider the system of Figure 4.1 with trustworthy relay nodes, i.e., $q_{n, r}=$ $0 \forall n \in \mathcal{N}, r \in \mathcal{R}$. The throughput optimal relay selection policy for this system was discussed in [48] and it was shown that the throughput optimal policy is equivalent to finding the maximum matching in a bipartite graph at each time slot. In the model considered in this work, we assume that the relay nodes may maliciously disobey the optimal scheduling algorithm and not forward the packets received in the first phase of cooperative transmission. In this case, such relay nodes behave like bumps in the network and cause larger average delay in the system. This causes the stability region of the network to shrink with respect to the system considered in [48]. In the following lemma we show that the stability region of the network with malicious relays shrinks with respect to the system considered in [48].

Lemma 1 Suppose that $\Lambda_{\text {Secure }}$ represents the stability region of a secure relaying system in which the trust values associated to all the relay nodes are 1 (All the elements of trust matrix $T$ are equal to 1$)$. Similarly, we denote the the stability region of a non-secure relaying system by $\Lambda_{\text {Non-Secure }}$ in which at least one element of matrix $T$ us structly less than 1 . Then,

$$
\Lambda_{\text {Non-Secure }} \subseteq \Lambda_{\text {Secure }}
$$


Proof: We first prove that $\Lambda_{\text {Non-Secure }} \subseteq \Lambda_{\text {Secure }}$ and then by an example we will show that in some cases $\Lambda_{\text {Non-Secure }} \subset \Lambda_{\text {Secure }}$. Consider the relaying system of Figure 4.1 in which $T_{n_{0}, r_{0}}<1$ for some $n_{0} \in \mathcal{N}$ and $r_{0} \in \mathcal{R}$ and other trust values are identically equal to 1 . We will compare this system with a secure system in which all the trust values are identically equal to 1 from the stability point of view.

We first characterize the stability region of a general relaying system introduced in Figure 4.1 which is denoted by $\Lambda$. A non-negative rate vector $\lambda=\left(\lambda_{1}, \lambda_{2}, \ldots, \lambda_{N}\right) \in \Lambda$ if there exist non-negative real elements $f_{n, r} \forall n \in \mathcal{N}, \forall r \in \mathcal{R}$ and $f_{n, D} \forall n \in \mathcal{N}$ such that

$$
\lambda_{n} \leq f_{n, D}+\sum_{r=1}^{R} f_{n, r} \quad \forall n \in \mathcal{N}
$$

and there exists a relay selection policy with indicator variables $I_{n, D}(t)$ and $I_{n, r}(t)$ for all $t$ such that

$$
\left\{\begin{array}{l}
f_{n, r} \leq \sum_{s \in \mathcal{S}} \pi_{s} T_{n, r} I_{n, r}(t) w_{n, r}^{(s)} \\
f_{n, D} \leq \sum_{s \in \mathcal{S}} \pi_{s} I_{n, D}(t) w_{n, D}^{(s)} \\
\sum_{n=1}^{N} I_{n, r}(t) \leq 1 \quad \forall r=1,2, \ldots, R \\
I_{n, D}(t)+\sum_{r=1}^{R} I_{n, r}(t) \leq 1 \quad \forall n=1,2, \ldots, N \\
I_{n, r}(t) \in\{0,1\}, I_{n, D}(t) \in\{0,1\} \quad \forall n \in \mathcal{N}, \forall r \in \mathcal{R}
\end{array}\right.
$$

in which $w_{n, r}^{(s)}$ and $w_{n, D}^{(s)}$ are the number of successful packet transmissions over the cooperative link $\ell_{n, r}$ and direct link $\ell_{n, D}$, respectively when the channel is in state $s$. From the above formulation of stability region and from the fact that $T_{n_{0}, r_{0}}<1$, it is concluded that $\Lambda_{\text {Non-Secure }} \subseteq \Lambda_{\text {Secure }}$. In the following, we will demonstrate that in some cases $\Lambda_{\text {Non-Secure }} \subset \Lambda_{\text {Secure }}$ by an example.

Example: Consider a relaying communication channel in which there is one source $S$, one relay node $r$ and one destination node $D$. We set $\frac{P}{W N_{0}}=20 d B$. The parameters $H_{S, D}(t), H_{S, r}(t)$ and $H_{r, D}(t)$ represent the effect of both location of users and relays 


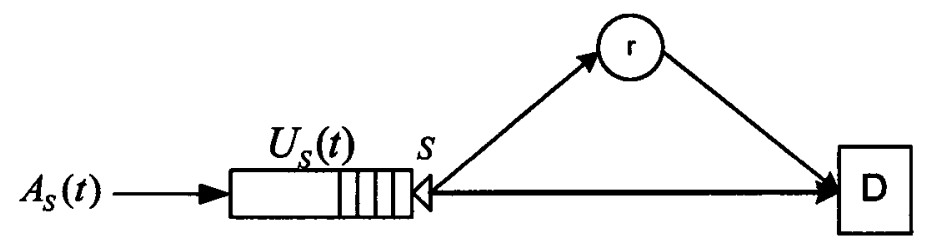

Figure 4.2: The model of cooperative communication used in the example

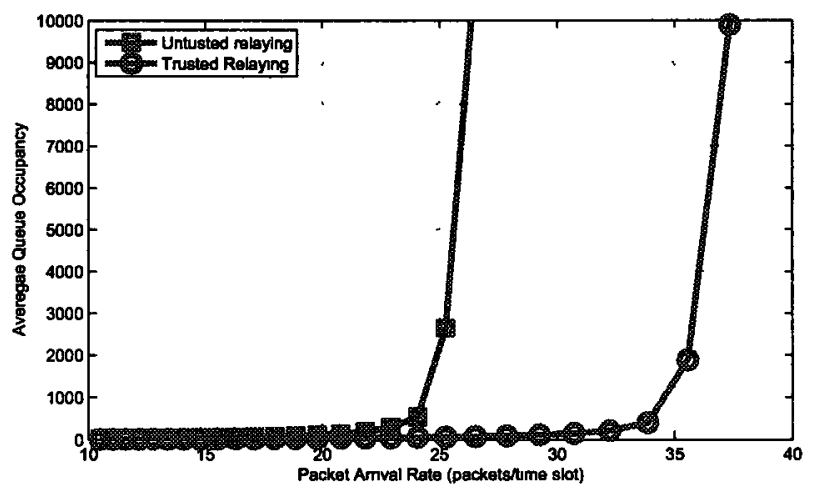

Figure 4.3: Comparison of stability region for trusted and untrusted relaying

as well as the shadowing and fading effects. Therefore, we may write them as

$$
H_{S, D}(t)=\frac{h_{S, D}(t)}{d_{S, D}^{\frac{2}{2}}(t)}, H_{S, r}(t)=\frac{h_{S, r}(t)}{d_{S, r}^{\frac{2}{2}}(t)}, H_{r, D}(t)=\frac{h_{r, D}(t)}{d_{r, D}^{\frac{a}{2}}(t)}
$$

where $d_{\imath, J}$ is the distance between nodes $i$ and $j$ and $\frac{a}{2}$ is the path loss exponent. We used $d_{S, r}=d_{r, D}=2 \sqrt{2}$ and $d_{S, D}=4$. The parameters $h_{\imath, j}(t)$ denote the effects of shadowing, fading, etc. at time slot $t$. We use $a=6$, and modelled $h_{\imath, j}(t)$ as i.i.d. multinomial random processes which takes the values $\{0.05,0.1,0.2,0.5\}$ with equal probabilities of 0.25 . The arrival processes to source node $S$ at each time slot are assumed to follow a Poisson distribution and packet length is 1024 bits. Packets of source node $S$ are kept in a queue to be transmitted in future. The source node $S$ transmits its packets with the cooperation of relay node $r$. Figure 4.2 illustrates the setting of the example. We compare two cases. In the first case, we will consider a trusted relay node and in the other case we will consider the system in which the relay node $r$ is malicious with probability 0.7 . We will compare the performance of the two systems in terms of stability region. Since in this example we just 
have one arrival stream of packets, stability region is determined by an inequality $\lambda \leq \lambda_{0}^{\text {Secure }}$ for trusted relaying system and $\lambda \leq \lambda_{0}^{\text {Non-Secure }}$ for untrusted relaying. Figure 4.3 depicts the result. As we can see the stability region of the untrusted relaying system is less than the stability region of trusted relaying. In other words, $\lambda_{0}^{\text {Non-Secure }}<\lambda_{0}^{\text {Secure }}$.

\subsection{Throughput-Optimal Secure Relay Selection Policy}

In this section, we will introduce the throughput optimal secure relay selection policy for the model described in section 4.1.1.

\subsubsection{Optimal Policy}

We introduce the relay selection policy $\Theta$ in which the indicator variables $I_{n, D}(t)$ and $I_{n, r}(t)$ are the solution to the following optimization problem.

Problem P:

$$
\begin{aligned}
\text { Maximize } & \sum_{n=1}^{N} U_{n}(t-1)\left(w_{n, D}(t) I_{n, D}(t)+\sum_{r=1}^{R} T_{n, r} w_{n, r}(t) I_{n, r}(t)\right) \\
\text { s.t. } & \sum_{n=1}^{N} I_{n, r}(t) \leq 1 \quad \forall r=1,2, \ldots, R \\
& I_{n, D}(t)+\sum_{r=1}^{R} I_{n, r}(t) \leq 1 \quad \forall n=1,2, \ldots, N \\
& I_{n, r}(t) \in\{0,1\}, I_{n, D}(t) \in\{0,1\} \quad \forall n \in \mathcal{N}, \forall r \in \mathcal{R}
\end{aligned}
$$

As we can see in the optimization problem $\mathbf{P}$, policy $\Theta$ determines the indicator variables $I_{n, D}(t)$ and $I_{n, r}(t)$ by considering the channel conditions, queue lengths and the trust associated to each relay node. In fact in policy $\Theta$, it is more probable to assign more secure relay nodes to users with better channel condition as well as larger queue backlogs. The following proposition states that the solution to the optimization problem $\mathbf{P}$ at each time slot determines the optimal decision variables 
$I_{n, D}(t)$ and $I_{n, r}(t)$ such that the stability of the system is guaranteed for all arrival rate vectors strictly inside the stability region.

Proposition 1 The relay selection polvcy $\Theta$ stabilizes the system for all the arrival rate vectors strictly inside the stability region $\Lambda$.

Proof: Let us introduce the following service processes:

- $\mu_{n, D}(t)$ : The amount of service provided at time slot $t$ over direct link $\ell_{n, D}$

- $\mu_{n, r}(t)$ : The amount of service provided at time slot $t$ over cooperative link $\ell_{n, r}$

Note that

$$
\mu_{n, D}(t)=w_{n, D}(t) I_{n, D}(t) \quad \text { and } \quad \mu_{n, r}(t)=w_{n, r}(t) I_{n, r}(t)\left(1-Q_{n, r}(t)\right)
$$

where $Q_{n, r}(t) \in\{0,1\}$ represents the maliciousness of relay node $r$ with respect to user $n$ at time slot $t$. The variable $Q_{n, r}(t)$ is equal to 1 when relay node $r$ does not cooperate with user $n$ at time slot $t$ and is equal to 0 otherwise. Obviously $E\left[Q_{n, r}(t)\right]=1-T_{n, r}$.

Since we have assumed that the channel state of the system is bounded and finite, therefore the processes $\mu_{n, D}(t)$ and $\mu_{n, r}(t)$ are bounded, i.e., $\mu_{n, D}(t) \leq \mu_{\max }<\infty$ and $\mu_{n, r}(t) \leq \mu_{\max }<\infty$. We will start with the Lyapunov drift evaluation. We will use the quadratic function $L(U(t))=\sum_{n=1}^{N} U_{n}^{2}(t)$ as the Lyapunov function. The Lyapunov drift $\Delta(t+1)$ for two successive time slots has the following form.

$$
\begin{aligned}
& \Delta(t+1) \leq E[L(U(t+1))-L(U(t)) \mid U(t))] \\
& \quad=E\left[\sum_{n=1}^{N} U_{n}^{2}(t+1)-U_{n}^{2}(t) \mid U(t)\right]
\end{aligned}
$$

Note that

$$
U_{n}(t+1)=\left(U_{n}(t)-\mu_{n, D}(t+1)-\sum_{r=1}^{R} \mu_{n, r}(t+1)\right)^{+}+A_{n}(t+1)
$$


It can be easily seen that

$$
\begin{aligned}
U_{n}^{2}(t+1)-U_{n}^{2}(t) & \leq A_{n}^{2}(t+1)+\left(\mu_{n, D}(t+1)+\sum_{r=1}^{R} \mu_{n, r}(t+1)\right)^{2} \\
& -2 U_{n}(t)\left(\mu_{n, D}(t+1)+\sum_{r=1}^{R} \mu_{n, r}(t+1)-A_{n}(t+1)\right)
\end{aligned}
$$

According to the fact that $\mu_{n, D}(t)+\sum_{r=1}^{R} \mu_{n, r}(t) \leq \mu_{m a x}$, the Lyapunov drift $\Delta(t+1)$ is written as

$$
\begin{aligned}
\Delta(t+1) & \leq \sum_{n=1}^{N} E\left[A_{n}^{2}(t+1)\right]+N \mu_{\max }^{2} \\
& +2 E\left[\sum_{n=1}^{N} U_{n}(t)\left(A_{n}(t+1)-\mu_{n, D}(t+1)-\sum_{r=1}^{R} \mu_{n, r}(t+1)\right) \mid U(t)\right] \\
= & \sum_{n=1}^{N} E\left[A_{n}^{2}(t+1)\right]+N \mu_{\max }^{2} \\
& +2 \sum_{n=1}^{N} U_{n}(t) \lambda_{n}-2 \sum_{n=1}^{N} E\left[U _ { n } ( t ) \left(w_{n, D}(t+1) I_{n, D}(t+1)\right.\right. \\
& \left.\left.-\sum_{r=1}^{R} w_{n, r}(t+1) I_{n, r}(t+1)\left(1-Q_{n, r}(t+1)\right)\right) \mid U(t)\right]
\end{aligned}
$$

$I_{n, d}(t+1)$ and $I_{n, d}(t+1)$ are valid indicator variables at time slot $t+1$ and are constrained to satisfy (4.6) and (4.7). Thus,

$$
\begin{aligned}
\Delta(t+1) & \leq \sum_{n=1}^{N} E\left[A_{n}^{2}(t+1)\right]+N \mu_{\max }^{2} \\
& +2 \sum_{n=1}^{N} U_{n}(t) \lambda_{n}-2 \sum_{n=1}^{N} U_{n}(t) E\left[w_{n, D}(t+1) I_{n, D}(t+1)\right. \\
& \left.-\sum_{r=1}^{R} T_{n, r} w_{n, r}(t+1) I_{n, r}(t+1) \mid U(t)\right]
\end{aligned}
$$

According to problem $\mathbf{P}$ and (4.18), we can see that policy $\Theta$ is minimizing the right hand side of (4.18). In other words, the right hand side of (4.18) for policy $\Theta$ is 
less than that of any other policy $\Theta^{\prime}$.

Now, consider the rate vector $\lambda=\left(\lambda_{1}, \lambda_{2}, \ldots, \lambda_{N}\right)$ strictly inside the stability region or inside the interior of stability region, i.e., $\lambda \in \operatorname{intr}(\Lambda)$ where $\operatorname{intr}(\cdot)$ is the interior of a set. Assume that $\epsilon_{N}$ is a vector of size $N$ whose elements are all identically equal to real number $\epsilon>0$ and $\epsilon$ is the largest positive real number such that $\lambda+\epsilon_{N} \in \Lambda$. Thus, there exists a relay selection policy $\Theta^{\prime}$ for which we have

$$
\lambda_{n}+\epsilon=E\left[w_{n, D}(t+1) I_{n, D}(t+1)+\sum_{r=1}^{R} T_{n, r} w_{n, r}(t+1) I_{n, r}(t+1)\right]
$$

and therefore

$$
\Delta^{\Theta^{\prime}}(t+1) \leq E\left[A_{n}^{2}(t+1)\right]+N \mu_{m a x}^{2}-2 \epsilon \sum_{n=1}^{N} U_{n}(t)
$$

According to (4.18), (4.19) and (4.20), we can conclude that

$$
\Delta^{\Theta}(t+1) \leq E\left[A_{n}^{2}(t+1)\right]+N \mu_{\max }^{2}-2 \epsilon \sum_{n=1}^{N} U_{n}(t)
$$

Based on Lyapunov drift argumentation, we can see that the right hand side of (4.21) would be negative if $\sum_{n=1}^{N} U_{n}(t) \geq \frac{E\left[A_{n}^{2}(t+1)\right]+N \mu_{\max }^{2}}{2 \epsilon}$ and this guarantees the stability of policy $\Theta$ for any arrival rate inside the interior of stability region.

\subsubsection{Bipartite Graph Representation of Optimal Policy}

Problem $\mathbf{P}$ has the general form of an integer programming problem. In general, integer programming problems are NP-hard. However, problem $\mathbf{P}$ has the structure of a maximum weighted matching problem in bipartite graphs [84]. The weighted bipartite graph associated to problem $\mathbf{P}$ is denoted by $G\left(V_{1}, V_{2}, E\right)$. Graph $G$ consists of two sets of vertices $V_{1}$ and $V_{2}$ and a set of edges $E$. Set $V_{1}$ is the set of all users and hence $\left|V_{1}\right|=N$. The set of vertices $V_{2}$ is defined as $V_{2}=\mathcal{R} \cup \mathcal{D}$ where $\mathcal{D}=\left\{D_{1}, D_{2}, \ldots, D_{N}\right\}$. Each node $D_{n} \in \mathcal{D}$ is associated to the direct transmission from user $n$. Therefore, in graph $G$ there is one edge between user $n \in V_{1}$ and $D_{n} \in V_{2}$ whose weight is $U_{n}(t) w_{n, D}(t)$. Therefore, each $D_{n} \in V_{2}$ is just connected to one node 


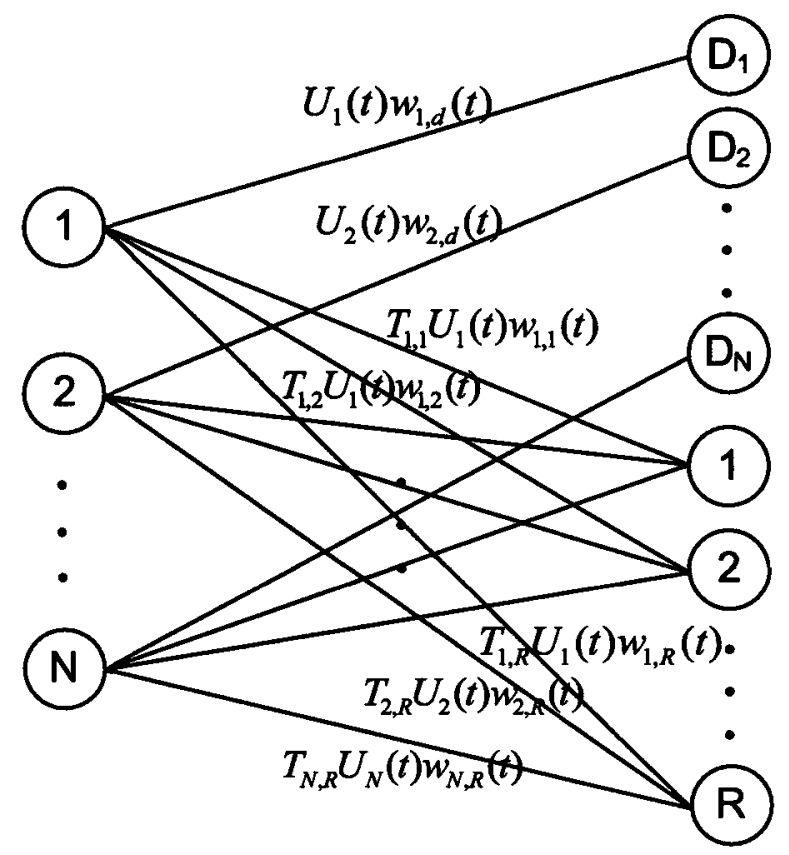

Figure 4.4: The bipartite graph representation of problem $\mathbf{P}$

$n \in V_{1}$. However, every node $n \in V_{1}$ is connected to all the nodes of $\mathcal{R} \subset V_{2}$. The weight of the edge connecting $n \in V_{1}$ and $r \in \mathcal{R}$ is equal to $T_{n, r} U_{n}(t) w_{n, r}^{D F}(t)$.

In summary, the edges weights $e$ are defined as the following:

- $e_{n, D_{n}}(t)=U_{n}(t) w_{n, D}(t) \forall n=1,2, \ldots, N$

- $e_{n, r}(t)=T_{n, r} U_{n}(t) w_{n, r}^{D F}(t) \forall n=1,2, \ldots, N \forall r=1,2, \ldots, R$

Figure 4.4 illustrates the structure of the bipartite graph $G$. A matching in the bipartite graph $G$ is a sub-graph of $G$ in which no two edges share a common vertex. We can see that the constraints of problem $\mathbf{P}$ impose that the solution to problem $\mathbf{P}$ must be a matching in graph $G$ and since the objective function in problem $\mathbf{P}$ is the summation of all the edges of graph $G$, the solution to problem $\mathbf{P}$ is a maximum weighted matching in graph $G$. Note that at each time slot, there may be more than one maximum weighted matching for graph $G$. In those cases, ties are broken arbitrarily.

Finding the maximum weighted matching in a bipartite graph is not NP-hard and has polynomial time algorithms. Hungarian algorithm is the most well known algorithm [81] to find the maximum matching in a bipartite graph whose complexity 
is of order $O\left(\left(\min \left\{\left|V_{1}\right|,\left|V_{2}\right|\right\}\right)\left(\max \left\{\left|V_{1}\right|,\left|V_{2}\right|\right\}\right)^{2}\right)$. This algorithm is used in our simulations to solve the optimization problem $P$. As we can see, the complexity order of this algorithm for the described model is $O\left(N(N+R)^{2}\right)$ which is a polynomial of order 3 with respect to $N$ and a polynomial of order 2 with respect to $R$. In other words, complexity of the algorithm increases faster when the number of users grow compared to the case where number of relays grow.

\subsection{Simulation Results}

In this section, we will compare the performance of policy $\Theta$ with the corresponding performance of four other policies using simulations. We call policy $\Theta$ as the Secure Throughput Optimal (STO) relay selection policy. Let us first introduce other four policies and then describe the simulation settings.

Consider the relay selection policy that assigns the relay nodes according to the solution of the following optimization problem.

$$
\begin{aligned}
\text { Maximize } & \sum_{n=1}^{N} U_{n}(t-1)\left(w_{n, D}(t) I_{n, D}(t)+\sum_{r=1}^{R} w_{n, r}(t) I_{n, r}(t)\right) \\
\text { s.t. } & \sum_{n=1}^{N} I_{n, r}(t) \leq 1 \quad \forall r=1,2, \ldots, R \\
& I_{n, D}(t)+\sum_{r=1}^{R} I_{n, r}(t) \leq 1 \quad \forall n=1,2, \ldots, N \\
& I_{n, r}(t) \in\{0,1\}, I_{n, D}(t) \in\{0,1\} \quad \forall n \in \mathcal{N}, \forall r \in \mathcal{R}
\end{aligned}
$$

Note that this policy is the policy used in [48] where it was assumed that all the relay nodes are trustworthy. In fact, this policy is equivalent to STO if $T_{n, r}=1 \forall n \in$ $\mathcal{N}, r \in \mathcal{R}$. We call this policy as Non-Secure Throughput Optimal (NSTO) relay selection policy since it does not consider the trust values when assigning the relay nodes.

Another policy considered in our simulations is the policy that does not consider queue length process in its assignments. In other words, the indicator variables $I_{n, D}(t)$ and $I_{n, r}(t)$ are determined by solving the following optimization problem. 


$$
\begin{aligned}
\text { Maximize } & \sum_{n=1}^{N} w_{n, D}(t) I_{n, D}(t)+\sum_{n=1}^{N} \sum_{r=1}^{R} T_{n, r} w_{n, r}(t) I_{n, r}(t) \\
\text { s.t. } & \sum_{n=1}^{N} I_{n, r}(t) \leq 1 \quad \forall r=1,2, \ldots, R \\
& I_{n, D}(t)+\sum_{r=1}^{R} I_{n, r}(t) \leq 1 \quad \forall n=1,2, \ldots, N \\
& I_{n, r}(t) \in\{0,1\}, I_{n, D}(t) \in\{0,1\} \quad \forall n \in \mathcal{N}, \forall r \in \mathcal{R}
\end{aligned}
$$

The objective of this policy is to maximize the secure instantaneous throughput at each time slot. Therefore, it is called Secure Instantaneous Throughput Optimal (SITO).

One way of securing the network with malicious relay nodes is to remove the malicious relay nodes from the set of relay nodes. In other words, we will not consider the relay nodes with $T_{n, r}<1$ in our allocation process. Therefore, we arrange a similar optimization problem to $\mathbf{P}$, but instead of using trust values of $T_{n, r}$ we will use $\left\lfloor T_{n, r}\right\rfloor$ in the objective function, i.e.,

$$
\begin{aligned}
\text { Maximize } & \sum_{n=1}^{N} U_{n}(t-1)\left(w_{n, D}(t) I_{n, D}(t)+\sum_{r=1}^{R}\left\lfloor T_{n, r}\right\rfloor w_{n, r}(t) I_{n, r}(t)\right) \\
\text { s.t. } & \sum_{n=1}^{N} I_{n, r}(t) \leq 1 \quad \forall r=1,2, \ldots, R \\
& I_{n, D}(t)+\sum_{r=1}^{R} I_{n, r}(t) \leq 1 \quad \forall n=1,2, \ldots, N \\
& I_{n, r}(t) \in\{0,1\}, I_{n, D}(t) \in\{0,1\} \quad \forall n \in \mathcal{N}, \forall r \in \mathcal{R}
\end{aligned}
$$

We call this policy as Secure Throughput Optimal with Pure Trusted Relays (STO-PTR). Note that in this policy, all the transmissions are secure since each user either transmits in direct mode or it uses pure trusted relay nodes for cooperation.

Last policy which is considered in our simulations is the non-cooperative (NC) policy in which all the users are just transmitting in direct mode. In this case, like 


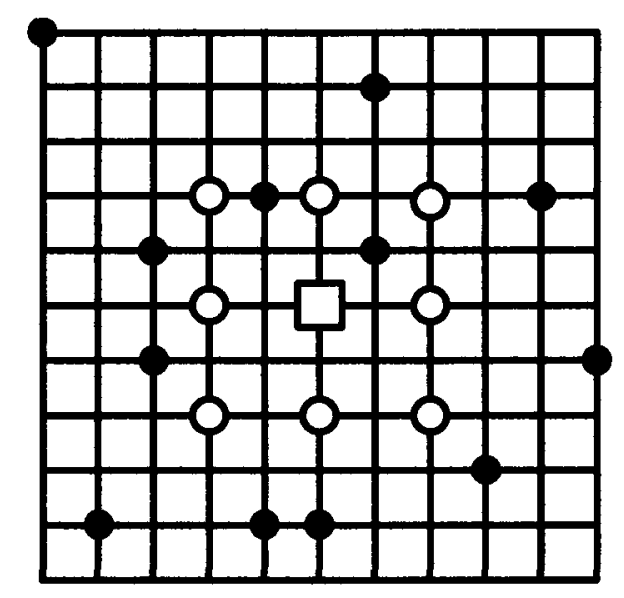

Figure 4.5: The grid cell structure

STO-PTR policy all the transmissions are secure transmissions. However, the users miss the opportunity to cooperate with relay nodes.

In our simulations, there are 12 users $(N=12)$ each equipped with one transceiver tuned on a separate orthogonal channel with the bandwidth of $W=100 \mathrm{KHz}$. We also considered 8 relay nodes $(R=8)$ in the system with fixed locations. All the nodes (users and relay nodes) are located in a grid cell structure with the size of $11 \times 11\left(\left[\begin{array}{ll}-5 & 5\end{array}\right] \times[-55]\right)$. The access point is located at the origin $(0,0)$. Figure 4.5 shows the cell structure used in the simulations.

The users are represented by black dots, the relay nodes by white circles and the access point is shown by a square in the middle. The users are initially distributed randomly on the grid but they can move with equal probabilities to the possible directions north, south, east and west at the beginning of each time slot. We do not allow a user to move to $(0,0)$ since the distance between the user and access point will be zero. We assume that relay 1 to 8 are located on $(2,0),(2,2),(0,2),(-2,2)$, $(-2,0),(-2,-2),(0,-2)$ and $(2,-2)$, respectively.

All the users and relay nodes transmit with the same level of power $P$. We will set $\frac{P}{W N_{0}}=20 d B$. The parameters $H_{i, j}(t), \forall i \in \mathcal{N} \bigcup \mathcal{R}, \forall j \in \mathcal{R} \bigcup D$ represent the effect of both location of users and relays as well as the shadowing and fading effects. Therefore, they can be written as

$$
H_{\imath, \mathcal{J}}(t)=\frac{h_{\imath, \jmath}(t)}{d_{\imath, \jmath}^{\frac{\jmath}{2}}(t)}
$$


where $d_{\imath, j}$ is the distance between nodes $i$ and $j$ and $\frac{a}{2}$ is the path loss exponent which is chosen to be 6 in our simulations. The parameters $h_{\imath, j}(t)$ denotes the effects of shadowing, fading, etc. at time slot $t$ and are modelled as i.i.d. multinomial random processes which takes the values $\{0.05,0.1,0.2,0.5\}$ with equal probabilities of 0.25 . With the above setting, the channel state information $C S I(t)$ evolves according to a finite state Markov chain. The arrival processes to all the queues at each time slot are assumed to follow a Poisson distribution and packet length is set to 1024 bits.

We considered four cases for which we plot the average total queue occupancy versus arrival rate.

In the first case we simulated a symmetric system in which the arrival rates to all the users are the same. Figure 4.6 illustrate the average total queue occupancy versus arrival rate. In the second case, we consider an asymmetric system in which we assume that the packet arrival rate to each user is proportional to its index. Therefore, if user 1 has arrival rate $\lambda$, user $i \geq 1$ has arrival rate $\imath \times \lambda$. We also assume that users $9,10,11$ and 12 are moving just on the border of the cell during the simulation. Figure 4.8 reflects the average total queue occupancy versus the arrival rate of user 1 (who has the smallest rate among all users). In both cases we assume that four of the relay nodes are trusted relays with trust values of 1 , i.e., $T_{n, r}=1 \forall n \in \mathcal{N}$ and for $r=2,4,6,8$. For other relay nodes, we assume that $T_{n, 1}=0.8, T_{n, 3}=0.6, T_{n, 5}=0.4$ and $T_{n, 7}=0.2 \forall n \in \mathcal{N}$.

In the third and the forth cases, we study the effect of trust values on the performance of each policy. Specifically, we consider two cases: a highly non-secure system with $T_{n, r}=0.2 \forall n \in \mathcal{N}$ and $\forall r \in \mathcal{R}$ and a relatively secure system with $T_{n, r}=0.8 \forall n \in \mathcal{N}$ and $\forall r \in \mathcal{R}$.

As we can observe from the figures, the stability region of STO policy is larger than all other policies in all the cases. In other words, the stable throughput region of the STO policy that we proposed is larger than other sub-optimal policies considered in the simulations. The reason to this fact is that STO considers the queue length process as well as trust values and channel conditions in the optimization $\mathbf{P}$. In fact, STO not only tries to maximize the service rate provided to the queue (by considering $w_{n, d}$ and $T_{n, r}$ ), but also it tries to balance the queues by multiplying those weights to the queue lengths and giving more resources to the users with very large backlogs. 


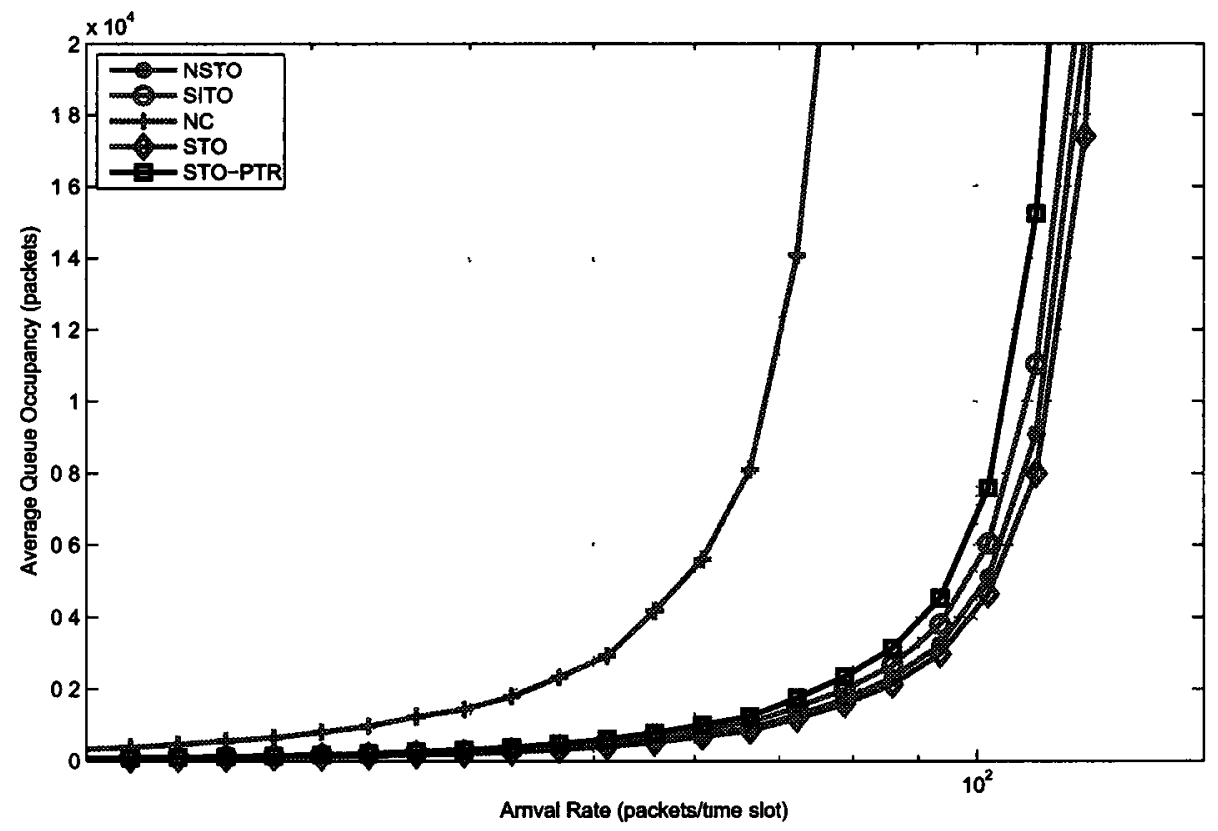

Figure 4.6: Average queue occupancy versus arrival rate in case 1

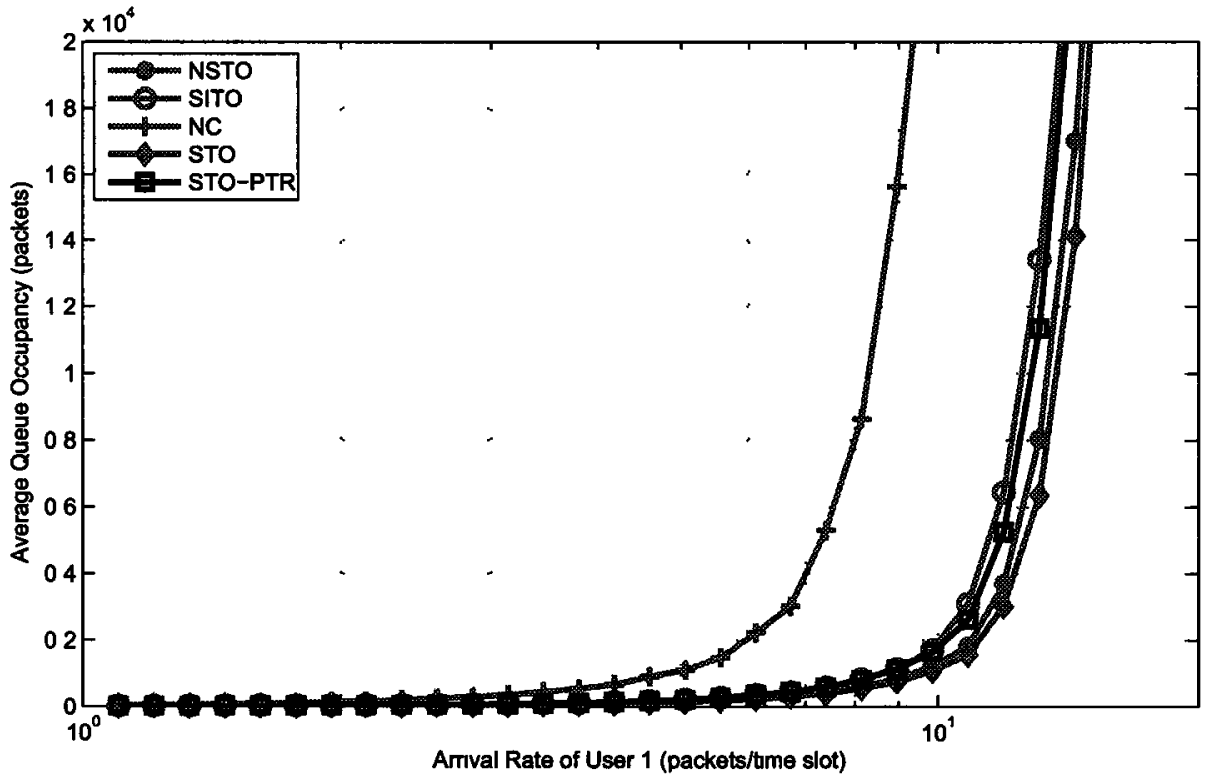

Figure 4.7: Average queue occupancy versus arrival rate in case 2 


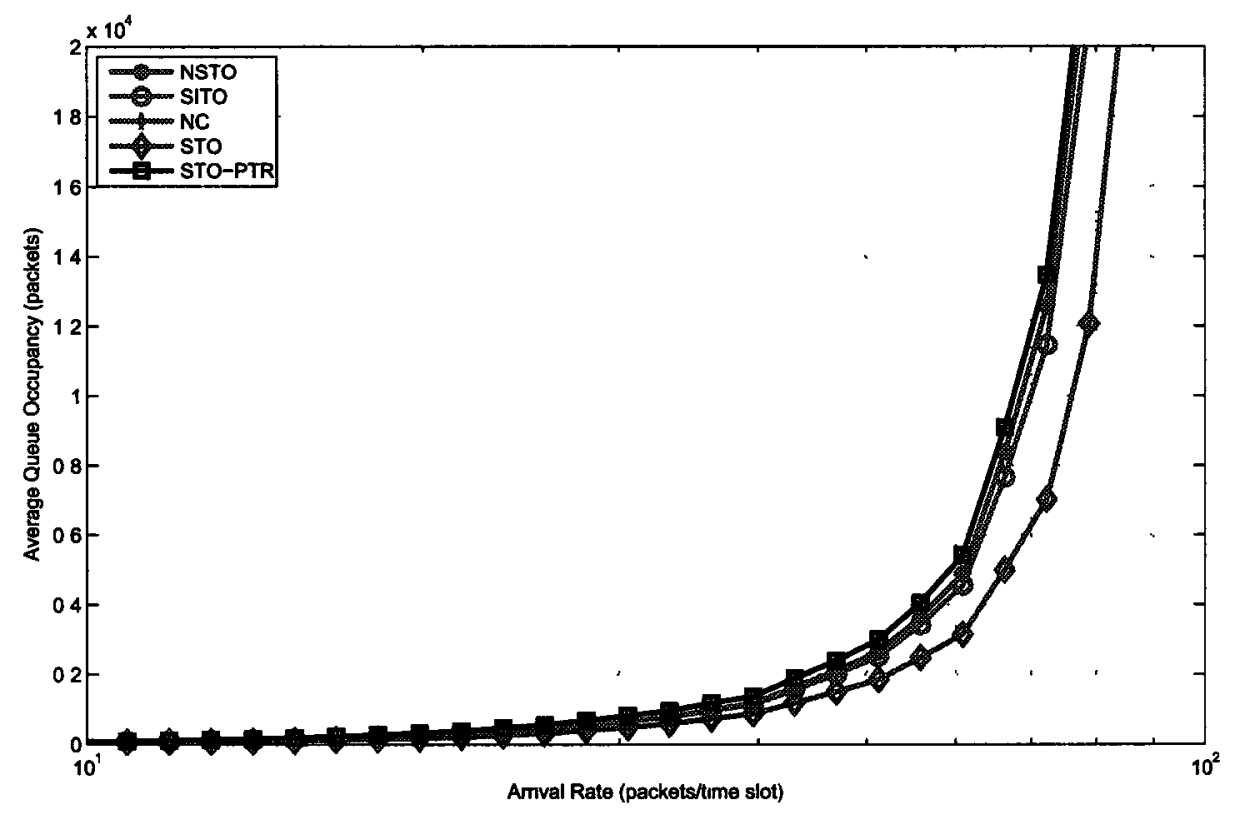

Figure 4.8: Average queue occupancy versus arrival rate in case 3

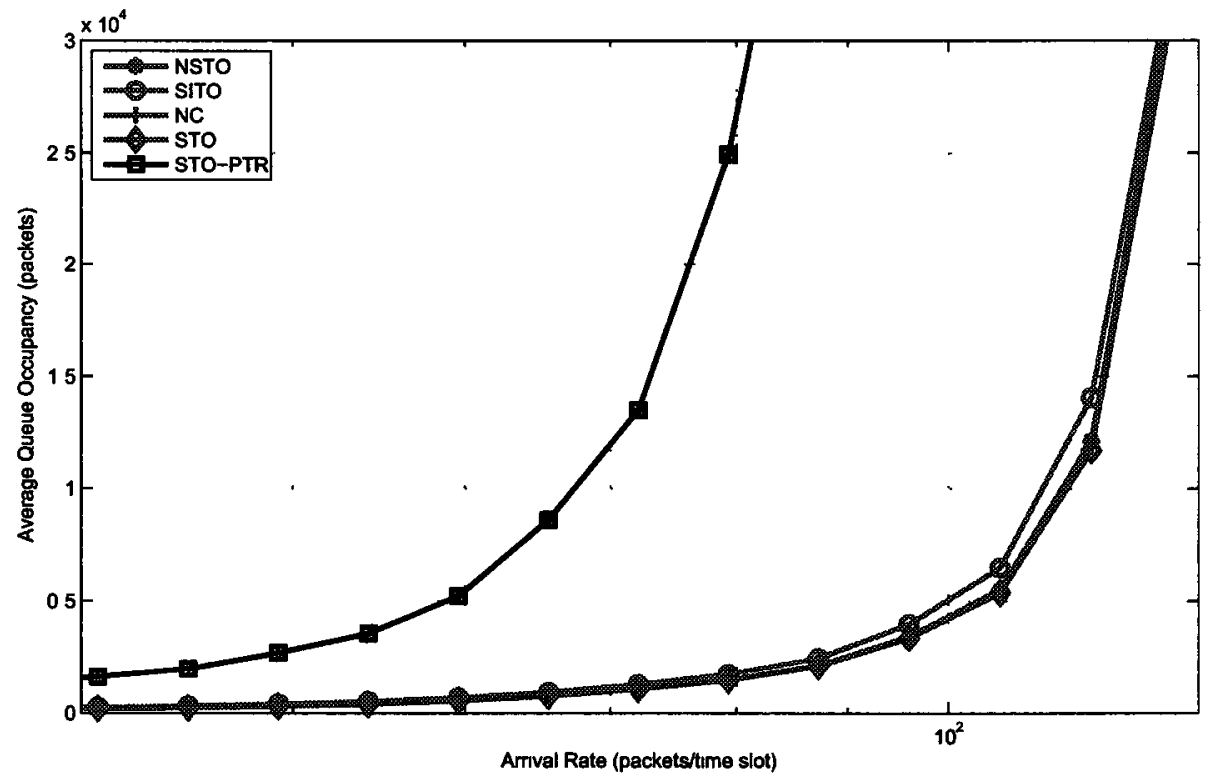

Figure 4.9: Average queue occupancy versus arrival rate in case 4 
We can observe that NC policy is always performing worse than other policies and the reason to this fact is that NC policy does not have the advantage of cooperation with relay nodes. In fact, cooperation can lead to significant improvement in the achievable rates and therefore can provide higher service rate for the queues.

As we compare the results in Figure 4.6 and 4.7, we can see that when the system is asymmetric the policy STO-PTR performs better than SITO. The reason is that in the asymmetric system, users with higher index have higher arrival rates as well as worse channel conditions and this results in higher backlog in those users. Therefore, balancing the queues would be very important to achieve higher stability region. Although STO-PTR removes relay nodes $2,4,6$ and 8 from the list of its relay nodes, its achieves higher stable throughput region with respect to SITO where queue length is not considered in relay selection decisions.

In Figures 4.8 and 4.9 we can see that two policies NC and STO-PTR perform the same. The reason to this fact is that in cases 3 and 4 of our simulations, all the relay nodes are malicious with positive probabilities and therefore in both cases there is no cooperative relaying. We also observe that as the trust values of the relays decrease, performance of all the policies that consider trust values in their relay selection decisions (STO,SITO,STO-PTR) and NC get closer to each other. In the extreme case where all the relay nodes are malicious with probability one, all these policies perform like NC policy. For trust value of 0.2 which was considered in our simulation, we see that the proposed policy STO performs better than all the policies and has larger stable throughput region. As the trust values get larger, the systems tends to more secure system and STO and NSTO performances get closer and are better than other policies specially NC and STO-PTR.

\subsection{Summary}

In this chapter, we introduced a throughput optimal relay selection policy for multiuser cooperative wireless networks. The proposed policy can stabilize the system for all the arrival rate vectors strictly inside the network stability region. We used Lyapunov stability analysis to prove the stability of the proposed method. We used simulations to compare the performance of the proposed policy with some other suboptimal cooperative and non-cooperative policies in terms of average backlog or equivalently average queueing delay. From the simulations we observed that the proposed 
throughput optimal policy outperforms other policies and has larger stable throughput region. 


\section{Chapter 5}

\section{Conclusions and Future works}

In this thesis, we reviewed the concept of trust and trust establishment in wireless networks. The related works were reviewed. In Chapter 3, we proposed a trust establishment scheme for wireless cooperative networks based on the Bayesian trust establishment framework. We considered a node as untrusted if it misuses the opportunity of the cooperation and does not forward the received packet when it is expected to do. First, by an example we showed that the conventional Bayesian trust establishment method is not able to estimate the relay nodes trust values precisely as they are biased on the channel quality of links as well as the relay selection policy. The proposed scheme tries to remove those biases to establish exact trust values for relay nodes.

The model that we considered consisted of a single source node, a destination node and a set of relay nodes. The relay nodes were assumed to be pure relay stations who do not inject traffic to the system. All nodes operate in half-duplex mode, i.e., they cannot transmit and receive the information simultaneously. The transmission paradigm from source to destination was a two-phase widely used transmission protocol in cooperative wireless networks. In the first phase of communication, source broadcasts a data packet to all relay nodes using a fixed amount of power while the relay nodes and destination are listening. In the second phase, one of the relay nodes which has successfully received the packet will be selected to forward the packet to the destination. The relay nodes were assumed to work in decode and forward mode. The amount of power was assumed to be fixed and the same for all relay nodes and the channels were assumed to be slow Rayleigh fading channels with additive white Gaussian noise. Based on the channel quality of the direct link from the source node to destination, two cases were considered. In the first case, the channel quality between 
the source and the destination was assumed to be poor. Therefore, the destination would not keep the received signal transmitted by source in the first phase for the purpose of combining. In the second case, the direct link between the source and the destination has an acceptable quality of channel. In contrast with the first case, in the second case the destination always would keep the received signal in the first phase of transmission to perform combining at the end of second phase. Other settings discussed above for the first case of the model was the same for the second case. The employed relay selection policy is to select the best relay node in terms of the available instantaneous channel state information of the system. Although a specific policy was used in the analysis, the results extend to any policy with the property of independence of relay selections from past and future relay selections. Note that the proposed method does not impose additional communication overhead to the system as it uses the available channel state information as well as relay selection decisions.

Through simulations, the effectiveness of our method was demonstrated. In the simulations, in an experiment we also showed the result of applying the proposed trust establishment method in a multi-user system using a different policy.

In the other part of this thesis, we devised an optimal relay selection policy in a relaying system in the presence of malicious relay nodes. We first showed that malicious behaviour of relay nodes may cause the stability region of the network to shrink. This motivated us to propose a throughput optimal secure relay selection policy which considers queue lengths, channel state and trust values associated to the relay nodes. We proved that the proposed policy is throughput optimal, i.e., it can stabilize the network for all the arrival rate vectors strictly inside the stability region. Note that the trust values employed in the proposed policy are general trust values which are real numbers from interval $[0,1]$ representing how much reliable is the relay node in cooperation with a specific user in terms of forwarding the received packets. The trust values could be the output of any intrusion detection or anomaly detection tool or trust establishment method.

In the studied model, there was a set of users, a set of relay nodes and a centralized controller controlling the relay assignment process. Each user could transmit directly to the destination or it could send its packets to the destination using the cooperation with a relay node according to a two phase cooperative protocol. Therefore, at each time slot, the network controller addressed two questions about each user: whether it should operate in the direct mode or cooperation mode; and if it has to 
cooperate, which relay node should be assigned to that user. The answers to these two questions determine a relay selection policy. We investigated the optimal policy for such a problem with the objective of maximizing the long run average throughput of the system. As mentioned earlier, the proposed optimal policy performs its duty based on the available information about the channel state of the system, trust values corresponding to each relay node as well as the length of each user's queue. This optimal policy was further characterized as an integer programming problem. Then, we showed that the optimal policy which we called it Secure Throughput Optimal (STO) is equivalent to determination of a maximum matching in a weighted bipartite graph at each time slot. Simulation was used to compare the performance of the proposed policy with four other sub-optimal relay selection policies in terms of average queue backlog or queueing delay and stability region. The other sub-optimal policies we considered for the comparison in our simulations include the following.

- NSTO: Non-Secure Throughput Optimal relay selection policy which does not consider the trust values when assigning the relay nodes to the users.

- SITO: Secure Instantaneous Throughput Optimal policy that does not consider queue length process in its assignments but considers trust values. The objective of this policy is to maximize the secure instantaneous throughput at each time slot.

- STO-PTR: Secure Throughput Optimal with Pure Trusted Relays which removes the malicious relay nodes from the set of relay nodes. In other words, the relay nodes whose trust value is less than 1 were not considered in the allocation process.

- NC: Non-Cooperative policy in which all the users were only transmitting in the direct mode without cooperation with any relay node.

In all the experiments, the stability region of STO policy is larger than all other policies in all the cases. The reason to this fact is that the proposed policy considers the queue length process as well as trust values and channel conditions in its optimization problem. In fact, this policy not only tries to maximize the service rate provided to the queues, but also it tries to balance the queues by incorporating the service rates and the queue lengths. Therefore, It gives more resources to the users with very large backlogs. 
Note that the relay selection policy proposed in Chapter 4 is a centralized control of relay assignment process in the network. Thus, in the future it will be worthwhile to investigate for distributed versions of the proposed policy which imposes less communication overhead to the network. Another interesting issue is to consider the system without the knowledge of trust values and instead estimate trust values adaptively as the system is running. Estimating the trust values can be achieved by the proposed trust establishment in Chapter 3. As the trust establishment speed is relatively high we expect to see very similar results. Moreover to extend the simulation results we can examine other channel models as well as mobility models. 


\section{List of References}

[1] A. Nosratinia, T. E. Hunter, and A. Hedayat, "Cooperative communication in wireless networks," IEEE Commun. Magazine, vol. 42, pp. 74-80, Oct. 2004.

[2] A. Sendonaris, E. Erkip, and B. Aazhang, "User cooperation diversity - part i: System description," IEEE Trans. Info. Theory, vol. 51, pp. 1927-1938, Nov. 2003.

[3] "IEEE standard for local and metropolitan area networks-part 16: Air interface for fixed broadband wireless access systems," IEEE Std.802.16-2004, 2004.

[4] D. Quercia, S. Hailes, , and L. Capra, "B-trust: Bayesian trust framework for pervasive computing," in Proc. 4th International Conference on Trust Management, (Pisa, Italy), May 2006.

[5] C. Zouridaki, B. L. Mark, M. Hejmo, and R. K. Thomas, "A quantitative trust establishment framework for reliable data packet delivery in manets," in Proc. 3rd ACM Workshop on Security of Ad Hoc and Sensor Networks (SASN'05)), (Alexandria, USA), Nov. 2005.

[6] L. Tassiulas and A. Ephremides, "Stability properties of constrained queueing systems and scheduling policies for maximum throughput in multihop radio networks," IEEE Trans. Auto. Control, vol. 37, pp. 1936-1949, Dec. 1992.

[7] G. Kramer, M. Gastpar, and P. Gupta, "Cooperative strategies and capacity theorems for relay networks," IEEE Trans. Inform. Theory, vol. 51, pp. 30373063, Sep. 2005.

[8] N. Laneman, D. Tse, and G. Wornell, "Cooperative diversity in wireless networks: Efficient protocols and outage behavior," IEEE Trans. Inform. Theory, vol. 50, pp. 3062-3080, Dec. 2004.

[9] L. Georgiadis, M. J. Neely, and L. Tassiulas, Resource Allocation and Cross Layer Control in Wireless Networks. Now Publisher, 2006.

[10] Y. Hong, W. Huang, F. Chiu, and C. C. J. Kuo, "Cooperative communications in resource-constrained wireless networks," IEEE Signal Processing Magazine, vol. 24, pp. 47-57, May 2007.

[11] J. G. Proakis, Digital Communications. 4th ed. McGraw-Hill, forth ed., 2001. 
[12] T. M. Cover and A. A. E. Gamal, "Capacity theorems for the relay channel," IEEE Trans. Inform. Theory, vol. 25, pp. 572-584, Sep. 1979.

[13] W. Ni, G. Shen, S. Jin, T. Fahldieck, and R. Muenzner, "Cooperative relay in IEEE 802.16j MMR," Tech. Rep. IEEE C802.16j-06_006r1, Alcatel, http://ieee802.org/16/relay/contrib/C80216j-06_006r1.pdf, May 2006.

[14] A. Sendonaris, E. Erkip, and B. Aazhang, "Increasing uplink capacity via user cooperation diversity," in Proc. of IEEE International Symposium on Information Theory, (Cambridge, USA), Aug. 1998.

[15] E. Beres and R. Adve, "Relay selection in cooperative networks," in Cooperative communications for improved wireless network transmission: Framework for virtual antenna array applications (M. Uysal, ed.), USA: Information Science Reference, 2010.

[16] M. Dohler and Y. Li, Cooperative communications: Hardware, Channel \& PHY. U.K.: John Wiley \& Sons, 2010.

[17] Y. Li, B. Vucetic, Z. Chen, and J. Yuan, "An improved relay selection scheme with hybrid relaying protocols," in Proc. of IEEE Global Telecommunications Conference (GLOBECOM'07), (Washington DC, USA), Nov. 2007.

[18] K. Chen and R. Prasad, Cognitıve radıo networks. U.K.: John Wiley \& Sons, 2009.

[19] R. U. Nabar, H. Bölcskei, and F. W. Kneubühler, "Fading relay channels: Performance limits and space-time signal design," IEEE Journal on Selected Areas in Commun., vol. 22, pp. 1099-1109, Aug. 2004.

[20] A. Avestimehr and D. Tse, "Outage capacity of the fading relay channel in the low-SNR regime," IEEE Trans. on Information Theory, vol. 53, pp. 1401-1415, Apr. 2007.

[21] J. Hu and N. Beaulieu, "Closed-form expressions for the outage and error probabilities of decode-and-forward relaying in dissimilar rayleigh fading channels," in IEEE International Conference on Communıcatıon. ICC'07, (Glasgow, U.K.), June 2007.

[22] Y. Zhao, R. Adve, and T. J. Lim, "Outage probability at arbitrary SNR with cooperative diversity," IEEE Commun. Letters, vol. 9, pp. 700-702, Aug. 2005.

[23] J. Hu and N. Beaulieui, "Performance analysis of decode-and-forward relaying with selection combining," IEEE Commun. Letters, vol. 11, pp. 489-491, June 2007.

[24] K. Hwang, Y. Ko, and M. Alouini, "Performance analysis of incremental relaying with relay selection and adaptive modulation over non-identically distributed cooperative paths," in Proc. of IEEE International Symposium on Information Theory, (Toronto, Canada), July 2008. 
[25] S. Ikki and M. Ahmed, "Performance analysis of incremental relaying cooperative diversity networks over rayleigh fading channels," in Proc. Conference on Wireless Communication and Networking. WCNC'08, (Las Vegas, USA), Apr. 2008.

[26] D. Djenouri, L. Khelladi, and N. Badache, "A survey of security issues in mobile ad hoc and sensor networks," IEEE Commun. Surveys \& Tutorials, vol. 7, no. 4, pp. 2-28, 2005.

[27] V. Stankovic, A. Host-Madsen, and Z. Xiong, "Cooperative diversity for wireless ad hoc networks," IEEE Signal Processing Magazine, vol. 23, pp. 37-49, Sep. 2006.

[28] A. Scaglione and Y. Hong, "Opportunistic large arrays: Cooperative transmission in wireless multihop ad hoc networks to reach far distances," IEEE Trans. on Signal Processing, vol. 51, Aug. 2003.

[29] A. Scaglione, D. L. Goeckel, and J. N. Laneman, "Cooperative communications in mobile ad hoc networks: Rethinking the link abstraction," in Distrubuted antenna systems: Open architecture for future wireless communicatıons (H. Yu, Y. Zhang, and J. Luo, eds.), USA: Auerbach Publications, 2007.

[30] A. Scaglione, D. Goeckel, and J. N. Laneman, "Cooperative communications in mobile ad hoc networks," IEEE Signal Processing Magazine, vol. 23, pp. 18-20, Sep. 2006.

[31] G. Kramer, I. Maric, and R. D. Yates, Cooperatıve Communıcatıons. Hanover MA: Foundations and Trends in Networking, NOW Publishers, 2007.

[32] B. Schein and R. G. Gallager, "The gaussian parallel relay network," in Proc. IEEE Int. Symp. Information Theory (ISIT), (Sorrento, Italy), June 2005.

[33] E. Beres and R. Adve, "Selection cooperation in multi-source cooperative networks," IEEE Trans. Wireless Commun., vol. 7, pp. 118-127, Jan. 2008.

[34] Y. Zhang, Y. Xu, and Y. Cai, "Relay selection utilizing power control for decodeand-forward wireless relay networks," in Proc. 2nd International Conference on Signal Processing and Communication Systems (ICSPCS 2008), (Gold Coast, Australia), Dec. 2008.

[35] Y. Jing and H. Jafarkhani, "Single and multiple relay selection schemes and their achievable diversity orders," IEEE Trans. Wireless Commun., vol. 8, pp. 14141423, March 2009.

[36] B. Wang, Z. Han, and K. J. R. Liu, "Distributed relay selection and power control for multiuser cooperative communication networks using stackelberg game," IEEE Trans. Mobile Comput., vol. 8, pp. 975-990, July 2009.

[37] S. Nam, M. Vu, and V. Tarokh, "Relay selection methods for wireless cooperative communications," in Proc. Conf. on Inform. Sciences and Systems (CISS), March 2008. 
[38] N. E. Wu, W. C. Huang, and H. J. Li, "A novel relay selection algorithm for relaying networks," in Proc. IEEE Vehıcular Technology Conference (Fall), (Anchorage, Alaska), Sep. 2009.

[39] Z. Yi and I. M. Kim, "Diversity order analysis of the decode and-forward cooperative networks with relay selection," IEEE Trans. Wireless Commun., vol. 7, no. 5 .

[40] A. Ibrahim, A. K. Sadek, W. Su, and K. J. R. Liu, "Cooperative communications with relay selection: When to cooperate and whom to cooperate with?," IEEE Trans. Wireless Commun., vol. 7, pp. 2814-2827, July 2008.

[41] A. K. Sadek, Z. Han, and K. J. R. Liu, "An efficient cooperation protocol to extend coverage area in cellular networks," in Proc. IEEE Wireless Communıcations and Networking Conference, (Las Vegas, NV), Apr. 2008.

[42] A. Bletsas, A. Lippman, and D. P. Reed, "A simple distributed method for relay selection in cooperative diversity wireless networks, based on reciprocity and channel measurements," in 61st IEEE Vehıcular Technology Conference (VTC'05), (Stockholm, Sweden), May 2005.

[43] Y. Zhao, R. S. Adve, and T. J. Lim, "Improving amplify-and-forward relay networks: optimal power allocation versus selection," IEEE Trans. on Wireless Commun., vol. 6, pp. 3114-3123, Aug. 2007.

[44] A. Bletsas, H. Shin, and M. Z. Win, "Outage probability at arbitrary SNR with cooperative diversity," IEEE Commun. Letters, vol. 11, pp. 261-263, March 2007.

[45] A. Bletsas, H. Shin, and M. Z. Win, "Cooperative communications with outageoptimal opportunistic relaying," IEEE Trans. on Wireless Commun., vol. 6, pp. 3450-3460, Sep. 2007.

[46] E. Yeh and R. Berry, "Throughput optimal control of cooperative relaying networks," IEEE Trans. Inform. Theory, special ıssue on Models, Theory and Codes for Relaying and Cooperation in Communication Networks, vol. 53, pp. 38273832, Oct. 2007.

[47] Y. Wei, F. R. Yu, M. Song, and V. C. M. Leung, "Energy efficient distributed relay selection in wireless cooperative networks with finite state markov channels," in Proc. IEEE Globecom 2009, (Honolulu, Hawaii, USA), Dec. 2009.

[48] H. Halabian, I. Lambadaris, C.-H. Lung, and A. Srinivasan, "Throughputoptimal relay selection in multiuser cooperative relaying networks," in IEEE MILCOM 2010, (San Jose, CA, USA), Nov. 2010.

[49] R. Urgaonkar and M. J. Neely, "Delay-limited cooperative communication with reliability constraints in wireless networks," in Proc. IEEE INFOCOM 2009, (Rio De Janeiro, Brazil), Nov. 2010.

[50] B. A. Forouzan, Cryptography and network security. USA: McGraw-Hill, 2008. 
[51] B. Wu, J. Chen, J. Wu, and M. Cardei, "A survey of attacks and countermeasures in mobile ad hoc networks," in Wireless network security (Y. Xiao, X. Shen, and D. Du, eds.), USA: Springer - Signals and Communication Technology, 2007.

[52] G. Schäfer, Security in fixed and wireless networks - An introductıon to securing data networks. U.K.: John Wiley \& Sons, 2003.

[53] L. Capra, "Toward a human trust model for mobile ad-hoc networks," in Proc. 2nd UK-UbıNet Workshop, (Cambridge, UK), May 2004.

[54] J. H. Cho, A. Swami, and I. R. Chen, "A survey on trust management for mobile ad hoc networks," IEEE Communications Surveys and Tutorials, pp. 1-22, 2010.

[55] H. Li and M. Singhal, "Trust management in distributed systems," IEEE computer, vol. 40, pp. 45-53, 2007.

[56] Y. L. Sun, Z. Han, F. Chiu, and K. J. R. Liu, "Information theoretic framework of trust modeling and evaluation for ad hoc networks," IEEE J. Sel. Areas Commun., vol. 24, no. 2, pp. 305-317, 2006.

[57] Y. Mao and M. Wu, "Security issues in cooperative communications: Tracing adversarial relays," in Proc. of IEEE International Conference on Acoustic, Speech, and Signal Processing. ICASSP'06, (Toulouse, France), May 2006.

[58] Y. Mao and M. Wu, "Tracing malicious relays in cooperative wireless communications," IEEE Trans. on Information Forensics and Securty, vol. 2, pp. 198-212, June 2007.

[59] X. He and A. Yener, "Two-hop secure communication using an untrusted relay: a case for cooperative jamming," in Proc. of IEEE Global Telecommunications Conference. GLOBECOM'08, (New Orleans, USA), Dec. 2008.

[60] E. Perron, S. Diggavi, and E. Telatar, "On cooperative wireless network secrecy," in Proc. IEEE INFOCOM 2009, (Rio de Janiero, Brazil), Apr. 2009.

[61] L. Lai and H. E. Gamal, "Cooperative secrecy: The relay-eavesdropper channel," in Proc. IEEE International Symposium on Information Theory. ISIT'07, (Nice, France), June 2007.

[62] S. Marti, T. Giuli, K. Lai, and M. Baker, "Mitigating routing misbehavior in mobile ad hoc networks," in Proc. of 6th ACM International Conference on Mobile Computing and Networking, (Boston, USA), Aug. 2000.

[63] S. Buchegger and J. Le Boudec, "Performance analysis of the confidant protocol," in Proc. of 3rd ACM International Symposium on Mobıle Ad hoc Networking $\mathcal{G}$ Computing, (Lausanne, Switzerland), June 2002.

[64] R. Mahajan, M. Rodrig, D. Wetherall, and J. Zahorjan, "Sustaining cooperation in multi-hop wireless networks," in Proc. of 2 nd ACM Conference on Symposium on Networked Systems Design 6 Implementation, (Boston, USA), May 2005. 
[65] A. Boukerche and Y. Ren, "A trust-based security system for ubiquitous and pervasive computing environments," Journal Computer Communications, vol. 31, no. 18, pp. 305-317, 2008.

[66] L. Dong, Z. Han, A. Petropulu, and H. Poor, "Improving wireless physical layer security via cooperating relays," IEEE Trans. Signal Processing, vol. 58, pp. 1875-1888, Mar. 2010.

[67] S. Makda, A. Choudhary, N. Raman, T. Korakis, Z. Tao, and S. Panwar, "Security implications of cooperative communications in wireless networks," in Proc. IEEE Sarnoff Symposium, (Princeton, USA), Apr. 2008.

[68] S. Dehnie, H. T. Sencar, and N. Memon, "Detecting malicious behavior in cooperative diversity," in Proc. Conference on Information Scrences and Systems. CISS'07, (Baltimore, USA), Mar. 2007.

[69] X. Jiang, C. Lin, H. Yin, Z. Chen, and L. Su, "Game-based trust establishment for mobile ad hoc networks," in Proc. 2009 WRI International Conference on Communucations and Mobıle Computing (CMC'09), Jan. 2009.

[70] R. Shankaran, V. Varadharajan, M. Orgun, and M. Hitchens, "Context-aware trust management for peer-to-peer mobile ad-hoc networks," in Proc. 33rd IEEE Computer Software and Applications Conference (COMPSAC'09), (Seattle, WA), July 2009.

[71] B. J. Chang and S. L. Kuo, "Markov chain trust model for trust-value analysis and key management in distributed multicast manets," IEEE Trans. Veh. Tech., vol. 58, pp. 1846-1863, Sep. 2009.

[72] S. Buchegger and J.-Y. L. Boudec, "A robust reputation system for $\mathrm{p} 2 \mathrm{p}$ and mobile ad-hoc networks," in Proc. 2nd Workshop on Economics of Peer-to-Peer Systems, (Boston, MA), June 2004.

[73] A. C. Davison, Statistical Models. London: Cambridge University Press, 2003.

[74] J. Berger, Statistical Decision Theory and Bayesıan Analysis. London: SpringerVerlag, 1985.

[75] Z. Han and Y. L. Sun, "Distributed cooperative transmission with unreliable and untrustworthy relay channels," EURASIP Journal on Wireless Communucations and Networking, special issue on Wireless Network Security, vol. 2009. Available: http://www.hindawi.com/journals/wcn/2009/740912.html, no. 11, 2009.

[76] R. Changiz, H. Halabian, F. R. Yu, I. Lambadaris, and H. Tang, "Trust management in wireless mobile networks with cooperative communications," in Proc. IEEE/IFIP TrustCom 2010, (HongKong, P.R. China), Dec. 2010.

[77] R. Changiz, H. Halabian, F. R. Yu, I. Lambadaris, H. Tang, and P. Mason, "Trust establishment in cooperative wireless networks," in IEEE Milcom 2010, (San Jose, CA, USA), Nov. 2010. 
[78] F. A. Onat, Y. Fan, H. Yanikomeroglu, and H. V. Poor, "Threshold based relay selection in cooperative wireless networks," in Proc. IEEE Globecom 2008, (New Orleans, LA), Nov. 2008.

[79] V. Sreng, H. Yanikomeroglu, and D. Falconer, "Relayer selection strategies in cellular networks with peer-to-peer relaying," in Proc. IEEE VTC'F03, (Orlando, FL), Oct. 2003.

[80] S. Dehnie and N. Memon, "A stochastic model for misbehaving relays in cooperative diversity," in Proc. IEEE WCNC 2008, (Las vegas, USA), 2008.

[81] H. W. Kuhn, "The hungarian method for the assignment problem," Naval Research Logistic Quarterly, pp. 83-97, 1955.

[82] L. Tassiulas and A. Ephremides, "Dynamic server allocation to parallel queues with randomly varying connectivity," IEEE Trans. Inform. Theory, vol. 39, pp. 466-478, March 1993.

[83] M. J. Neely, Dynamic power allocation and routing for satellite and wireless networks with time varyıng channels. MIT,USA: Ph.D. dissertation, Massachusetts Institute of Technology, LIDS, 2003.

[84] T. H. Cormen, C. E. Leiserson, R. L. Rivest, and C. Stein, Introductıon to Algorithms. USA: MIT Press, 2001. 


\section{Appendix A}

\section{Simulation Programs}

\%This Function receives the arrival rate to the single user system with out combining $\%$ pa, and $N$ iterations as input and calculates the trust values of $M$ relay Nnde with window size w. This Function invokes SNR.m function and Produce.m function

function wo_comb_ $\operatorname{Tr}(\mathrm{pa}, \mathrm{N})$

$\%$ gama1_avg=[10^5.2 10^4.9 10^5.4 10^5]; \%Sample vector of SNRs between $S$ and $r_{-} \imath$ in Low SNR regieme

$\%$ gamaZ_avg $=\left[10^{\wedge} 610^{\wedge} 710^{\wedge} 6.310^{\wedge} 6.5\right]$; \%Sample vector of SNRs between $r_{-} \imath$ and $D$ in High SNR regıeme

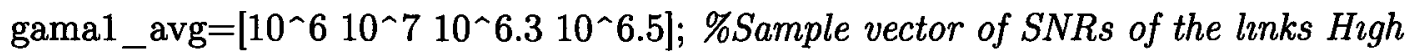
SNR regieme

gama2_avg $=\left[10^{\wedge} 610^{\wedge} 710^{\wedge} 6.310^{\wedge} 6.5\right]$

$\mathrm{T}=30 ; \%$ Calculations are pefromed $\mathrm{T}$ times to be accurate

$\mathrm{M}=4 ; \%$ Number of relays

$\mathrm{w}=50 ; \%$ Window size

Frame $=100 ; \%$ Number of frames observed in an iteration

ts_avg $=\operatorname{zeros}(\mathrm{M}, \mathrm{N}) ; \%$ Proposed trust values which will be averaged in $T$ runs

tz_avg $=$ zeros $(\mathrm{M}, \mathrm{N}) ; \%$ Conventional Bayesian trust values

for $\mathrm{t}=1: \mathrm{T}$

$\mathrm{q}=\left[\begin{array}{llll}0.1 & 0.2 & 0.3 & 0.4\end{array}\right] ;$ \% Vector of Probabulutres of malıcrousness

$\%$ Initial Values

select $=\mathbf{z e r o s}(1, \mathrm{M}) ; \%$ Number of times in which the relay is selected in an iteration

$\mathrm{N}_{\text {_g_rd=zeros }}(1, \mathrm{M}) ; \%$ Number of times in which the relay has acceptable quality of channel to $D$

p_sel $=0.5$.*ones $(1, \mathrm{M}) ;$ \%estrmation of probability of a relay berng selected

P_SNR_rd=0.5.*ones $(1, \mathrm{M})$; \%estımation Probabiluty of having good quality channel between relay to $D$

$\mathrm{N}$ _selection $=0 ; \%$ Number of times when relay selection us performed.

$\mathrm{mal}=\mathbf{z e r o s}(1, \mathrm{M}) ;$ \%vector maliciousness of $M$ relays

gama_Th $=10^{\wedge} 5.4 ; \% E b / N O=54$ for bpsk ber $=10^{\wedge}-6=>$ Threshold $S N R=54 \mathrm{~dB}$

que $0=1 ; \% Q u e u e$ length at user side 
\%Proposed Trust parameters for the case with out combining alpha_wo_p=ones $(1, \mathrm{M})$;

beta_wo_p=ones $(1, \mathrm{M})$;

\% Connectivity

$\mathrm{CR}=\mathbf{z e r o s}(1, \mathrm{M}) ; \%$ Connectivity vector of links between relays to $D$ gamaL $=\operatorname{zeros}(1, \mathrm{M}) ; \% S N R$ vector of links between source and relays gamaR=zeros $(1, \mathrm{M}) ; \% S N R$ vector of links between relays and $D$

\%Conventional Bayesian Trust Parameters of Beta function

alpha_Bayes=ones $(1, \mathrm{M})$;

beta_Bayes=ones $(1, \mathrm{M})$;

\%Trusts

$\mathrm{tz}=\mathrm{zeros}(\mathrm{M}, \mathrm{N})$;

ts $=\operatorname{zeros}(\mathrm{M}, \mathrm{N})$;

counter $=$ zeros $(1, \mathrm{M}) ; \%$ Times which relay has received a packet and has an \%acceptable SNR to $D$ and

sent $=$ zeros $(\mathrm{N}, \mathrm{M}) ; \%$ Number of successful received packets at $D$

recv $=$ zeros $(\mathrm{N}, \mathrm{M}) ; \%$ Number of packets received at relays

for $\mathrm{j}=1: \mathrm{N} \%$ Start of teration $\mathrm{j}$

for $\mathrm{i}=1$ :Frame \%Start of frame $\imath$

Broadcasted $=0$;

\%slot 1 starts with calculating arrivals useing Produce.m

$\mathrm{a}=$ produce $(\mathrm{pa})$;

que $0=$ que $0+a ;$

if que $0>0$

$\mathrm{CL}=\operatorname{zeros}(1, \mathrm{M}) ; \%$ Connectivities from $S$ to relays

\%calculatıng SNRs from $S$ to relay $k$ based on the

\%gama1_avg using SNR.m

\%Calculatıng Number of received packets at relays

for $\mathrm{k}=1: \mathrm{M}$

$\operatorname{gamaL}(\mathbf{k})=\mathrm{SNR}($ gama1_avg $(\mathbf{k}))$;

if gamaL(k)>gama_Th

$\mathrm{CL}(\mathrm{k})=1$;

Broadcasted $=1$;

$\operatorname{recv}(\mathrm{j}, \mathrm{k})=\operatorname{recv}(\mathrm{j}, \mathrm{k})+1$;

end

end

\%slot 2:

$\mathrm{a}=\operatorname{produce}(\mathrm{pa})$

que $0=$ que $0+a ;$

\%If at least one relay recieved the signal selection is

\%performed

if $($ Broadcasted $==1)$,

que $0=$ que $0-1$

\%Relay Selection:

$\mathrm{CR}=\operatorname{zeros}(1, \mathrm{M})$;

\%Determining the tımes when a relay has 
\%acceptable SNR from relay $k$ to $D$

for $\mathrm{k}=1: \mathrm{M}$

$\operatorname{mal}(\mathbf{k})=\operatorname{produce}(\mathrm{q}(\mathrm{k})) ; \%$ Malıcıousness $R V$ are produced with parameter $q(k)$

$\operatorname{gamaR}(\mathrm{k})=\mathrm{SNR}($ gama2_avg(k));\%SNR of the links from relay to $D$ is produced

if gamaR(k)>gama_Th \%Compare to Threshold SNR

$\mathrm{CR}(\mathrm{k})=1 ; \%$ connectivity

if $\mathrm{CL}(\mathrm{k})==1$

counter $(\mathrm{k})=$ counter $(\mathrm{k})+1 ;$ \%relay has recevved and has acceptable SNR

end

N_g_rd(k)=N_g_rd(k)+1; \%Number related to relay having \%acceptable SNR to D

end

end

sel_set=gamaR.*CL;

$[\mathrm{g}, \overline{\mathrm{r}}]=\max \left(\mathrm{sel} \_s e t\right) ; \%$ Selectıng Relay With Best channel

if $\mathrm{CR}(\mathrm{r})>0$

select(r) $=$ select(r) $+1 ; \%$ Number of times being selected

end

if $(\mathrm{mal}(\mathrm{r})==0) \& \&(\mathrm{CR}(\mathrm{r})==1)$

$\operatorname{sent}(\mathrm{j}, \mathrm{r})=\operatorname{sent}(\mathrm{j}, \mathrm{r})+1 ; \%$ Number recevved packets at $D$ end

end

end

$\mathrm{N}_{\text {_selection }}=\mathrm{N}$ _selection $+1 ; \% \mathrm{~N}$ of relay selections

end \%A frame ends

\%calculations proposes trust for Relays

P_SNR_rd=N_g_rd./N_selection; \% Estımatıng the probability of havıng an acceptable $\overline{S N} \bar{R}$ from relay to $D$

if $\mathrm{j}>\mathrm{w} \%$ calculations of the trust using a sliding window

alpha_wo_p=alpha_wo_p+sent $(\mathrm{j},:)-\operatorname{sent}(\mathrm{j}-\mathrm{w},:) ; \%$ \%rscards the number of packets before the strat of sluding window

beta_wo_p=beta_wo_p $+\operatorname{recv}(j,:)-\operatorname{recv}(j-w,:)-\operatorname{sent}(j,:)+\operatorname{sent}(j-W,:)$; else

alpha_wo_p=alpha_wo_p+sent $(j,:)$;

end

beta_wo_p=beta_wo_p+recv $(j,:)-\operatorname{sent}(j,:)$;

ts $(:, j)=$ alpha_wo_p./(alpha_wo_p+beta_wo_p)./P_SNR_rd./p_sel; $\%$ Proposed Trust Value

p_sel=select./(counter); \%update Probabılity of berng selected

ts_avg $(:, \mathrm{j})=\mathrm{ts} \_\operatorname{avg}(:, \mathrm{j})+\mathrm{ts}(:, \mathrm{j})$;

\% Conventional Bayesian trust parameters are calculated and then updated 
alpha_Bayes $=$ alpha_Bayes $+\operatorname{sent}(\mathrm{j},:)$;

beta_Bayes=beta_Bayes $+\operatorname{recv}(j,:)-\operatorname{sent}(j,:)$;

$\mathrm{tz}(:, \mathrm{j})=$ alpha_Bayes./(alpha_Bayes+beta_Bayes); \%trusts are calculated

$\mathrm{tz} \_\operatorname{avg}(:, \mathrm{j})=\overline{\mathrm{tz}}_{-} \operatorname{avg}(:, \mathrm{j})+\mathrm{tz}(: \overline{\mathrm{j}}) ; \%$ Sum of the trusts to be averaged

end $\overline{\%}$ Iteration ends

end \%Run $T$ has been performed

\%calculating the average trusts

ts_avg $=$ ts_avg. $/ \mathrm{T}$;

tz_avg=tz_avg./T;

end

\% This Function, SNR.m generates exponentıal distributed random numbers

$\%$ with mean gama_avg

function $\mathrm{U}=\mathrm{SNR}$ (gama_avg)

$\mathbf{u}=$ unifrnd $(0,1,1,1) ; \%$ generation of a random number in $[0,1]$

$\mathrm{U}=\operatorname{expinv}(\mathrm{u}, \mathrm{gama}$ _avg);

end \%function en $\bar{d}$

$\%$ This function generates a number which is 1 with probability $p$

$\%$ and is 0 otherwise.

function $\mathrm{y}=$ produce $(\mathrm{p})$

$\mathrm{u}=\mathrm{unifrnd}(0,1,1,1) ; \%$ generation of a random number in $[0,1]$

if $\mathrm{u}<\mathrm{p}$

$y=1$;

else

$y=0$

end

end

\%This Function Recevves the arrival rate to the user/users in the system with combining

$\%$ as input and calculates the trust values of $M$ relay Node with respect to $n$ users in $N$ iterations.

\% It saves the 3 dimentional trust matrix as a result whose dimentions are (user,relay, iteration)

function comb_multi(pa,N)

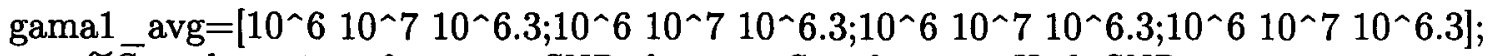

$\% \overline{S a m p l e}$ vector of average SNRs between $S$ and $r_{-} \imath$ in High SNR regieme

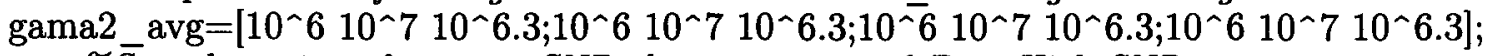

$\% \overline{S a m p l e}$ vector of average SNRs between $r_{2} \imath$ and $D$ in High SNR regieme

gama_d_avg $=\left[10^{\wedge} 510^{\wedge} 510^{\wedge} 510^{\wedge} 510^{\wedge} 5\right] ; \% \overline{V e c t o r}$ of average SNRs from users to $D$

$\mathrm{U}=4 ; \overline{\%} \overline{N u}$ mber of users

$\mathrm{M}=3 ; \%$ Number of relays

$\mathrm{T}=30 ; \%$ Number of time the calculation are performed for accuracy

Frame $=100 ; \%$ Frame size

tco_avg=zeros $(\mathrm{U}, \mathrm{M}, \mathrm{N})$; 
for $\mathrm{t}=1: \mathrm{T}$

tco $=\operatorname{zeros}(\mathrm{U}, \mathrm{M}, \mathrm{N}) ; \%$ Proposed trusts

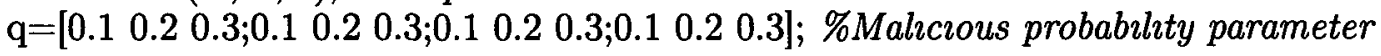
matrix

$\%$ estimation of probability of a relay being selected

p_sel=zeros $(\mathrm{U}, \mathrm{M})$;

\%\% estrmation Prob. of having good channel

$\mathrm{p}_{\text {_co }}=\mathrm{zeros}(\mathrm{U}, \mathrm{M})$;

\%how many times selection is peformed

que $=$ ones $(1, \mathrm{U})$

mal=zeros $(\mathrm{U}, \mathrm{M}) ; \%$ malıciousness of relay $M$ for user $U$

gama_Th $=10^{\wedge} 5.4 ; \% E b / N O=54$ for bpsk ber $=10^{\wedge}-6=>$ Threshold $S N R=54 d B$

\%mult-user system trustparameters proposed mehthod

alpha_co=ones(U,M); \%Cooperatıve channel SNR estımatıon parameters

beta_co=ones $(\mathrm{U}, \mathrm{M})$;

alpha_g_co=ones(U,M);\%Channel estımation of links between user $u$ and relay

beta_g_co $=$ ones $(\mathrm{U}, \mathrm{M})$;

$\%$

alpha_se_co=ones $(\mathrm{U}, \mathrm{M})$; Number of times a relay has been selected is estimated by these parameters

beta_se_co=ones $(\mathrm{U}, \mathrm{M})$;

\%connectivity

C_co=zeros $(\mathrm{U}, \mathrm{M}) ; \%$ Connectivity vector of links between relays to $D$

gamaL $=\operatorname{zeros}(\mathrm{U}, \mathrm{M}) ; \% S N R$ vector of links between user $n$ and relays

gamaR=zeros $(\mathrm{U}, \mathrm{M}) ; \% S N R$ vector of links between relays and $D$

gamaL=zeros $(1, \mathrm{M}) ; \% A v g$. SNR vector of links between source and relays

gamaR=zeros $(1, \mathrm{M}) ; \%$ Avg. SNR vector of links between relays and $D$

gama_d=zeros $(1, \mathrm{U}) ; \% A v g$. SNR of user $n$ to $D$

$\%$ Trusts

for $\mathrm{j}=1: \mathrm{N} \%$ iteratıon 3 starts

select $=\operatorname{zeros}(\mathrm{U}, \mathrm{M}) ; \%$ Number of times in which the relay is selected in an iteration est_co=zeros $(\mathrm{U}, \mathrm{M}) ; \%$ Number of time that cooperative channel has acceptable $S N R$

counter $=$ zeros $(\mathrm{U}, \mathrm{M}) ; \%$ Number of times in which the relay can be selected

$\mathrm{N}_{\text {_selection}=z e r o s}(1, \mathrm{U}) ; \% N$ of observation on each iteration when at least a relay recerves the message

sent $=\mathbf{z e r o s}(\mathrm{U}, \mathrm{M}) ; \%$ Number of successful received packets at $D$

$\mathrm{recv}=\mathrm{zeros}(\mathrm{U}, \mathrm{M}) ; \%$ Number of packets recelved at relays

for $\mathrm{i}=1: 100 \%$ FRAME START

\%slot 1

Broad $=\mathbf{z e r o s}(1, \mathrm{U}) ;$ \%variable indicaing that at least a relay has received the users signal

for $\mathrm{u}=1: \mathrm{U}$ \%for users compute

$\mathrm{a}(\mathrm{u})=$ produce $(\mathrm{pa}) ; \%$ Arrvvel with mean $p a$ 
que $(u)=q u e(u)+a(u)$

if que $(u)>0$

$\mathrm{CL}(\mathrm{u},:)=\operatorname{zeros}(1, \mathrm{M}) ; \%$ connectivity from user $u$ to relay for $k=1: M$

$\operatorname{gamaL}(\mathrm{u}, \mathrm{k})=\mathrm{SNR}($ gama1_avg $(\mathrm{u}, \mathrm{k})) ; \%$ Generating $S N R$ s from $S$ to relay $k$ based on the

\%gama1_avg using SNR.m

if $\operatorname{gamaL}(\mathrm{u}, \mathrm{k})>=$ gama_Th $\%$ Channel between $u$ and relay $k$ is acceptable

$\mathrm{CL}(\mathrm{u}, \mathrm{k})=1$;

$\operatorname{Broad}(\mathrm{u})=1$;

$\operatorname{recv}(\mathrm{u}, \mathrm{k})=\operatorname{recv}(\mathrm{u}, \mathrm{k})+1 ; \%$ If relay $k$ has recevved message of user $u$ end

end

gama_d $(\mathrm{u})=\mathrm{SNR}($ gama_d_avg $(\mathrm{u})) ; \%$ Generate SNR of the link $u-D$

\%slot 2

$\mathrm{a}(\mathrm{u})=$ produce $(\mathrm{pa}) ; \%$ Possible arrival

que $(u)=q u e(u)+a(u)$;

if $\operatorname{Broad}(\mathrm{u})==1 \%$ If a relay has recevved

$\mathrm{N}_{\text {_selection }}(\mathrm{u})=\mathrm{N}_{\text {_ }} \operatorname{selection}(\mathrm{u})+1$;

\%Relay Selection:

C_co $(\mathrm{u},:)=\operatorname{zeros}(1, \mathrm{M})$;

for $\mathrm{k}=1: \mathrm{M}$

\%Malıcrousness

$\operatorname{mal}(\mathrm{u}, \mathrm{k})=\operatorname{produce}(\mathrm{q}(\mathrm{u}, \mathrm{k})) ; \%$ Generating malıcıousness $R V$ of relay $k$ with respect to user $u$ )

$\operatorname{gamaR}(\mathrm{u}, \mathrm{k})=\mathrm{SNR}($ gama2_avg $(\mathrm{u}, \mathrm{k})) ; \% S N R$ of the links from relay to

$D$ is produced

if gamaR(k)>gama_Th \%Compare to Threshold SNR for the

purpose of channel estimating

gama_co(u,k)=gamaR $(\mathrm{u}, \mathrm{k})+$ gama_d $(\mathrm{u}) ; \%$ Cooperatıve $S N R$

$\%$ Af received counter is increased

if gama_co(u,k)>=gama_Th

C_co(u, $\mathrm{k})=1 ; \%$ Acceptäble cooperatıve channel beteen user $u$ and $D$

if $\mathrm{CL}(\mathrm{u}, \mathrm{k})==1$

$\%$ counter for relay selection $p$

counter $(\mathrm{u}, \mathrm{k})=$ counter $(\mathrm{u}, \mathrm{k})+1 ; \%$ relay could be selected

end

est_co $(\mathrm{u}, \mathrm{k})=\mathrm{est}$ co $(\mathrm{u}, \mathrm{k})+1 ; \%$ Number related to relay having

\%acceptable SNR of cooperative channel end

end

end

end

end 


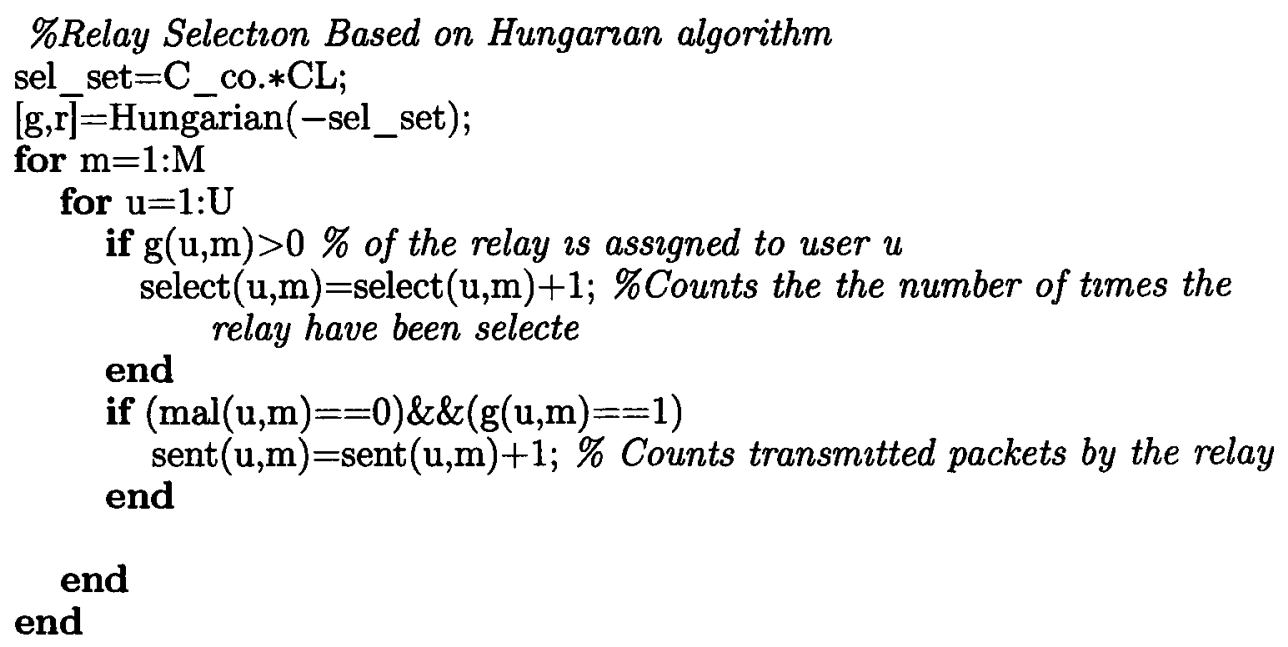

end

for $\mathrm{m}=1: \mathrm{M} \%$ relays

for $\mathrm{u}=1: \mathrm{U} \%$ users

p_sel $(\mathrm{u}, \mathrm{m})=$ alpha_se_co $(\mathrm{u}, \mathrm{m}) /($ alpha_se_co $(\mathrm{u}, \mathrm{m})+$ beta_se_co $(\mathrm{u}, \mathrm{m})) ; \%$ Estimation of $\overline{n u m b e r}$ of assignment of relay $m$ to user $u$

p_co $(\mathrm{u}, \mathrm{m})=$ alpha_g_co $(\mathrm{u}, \mathrm{m}) /(\mathrm{alpha}$ _g_co $(\mathrm{u}, \mathrm{m})+\mathrm{beta}$ _g_co $(\mathrm{u}, \mathrm{m})) ; \%$ Channel estımation Parameters

end

end

$\operatorname{tco}(:,,, \mathrm{j})=$ alpha_co./(alpha_co+beta_co $). /$ p_sel./p_co; \%Proposed Trust Value main formula

alpha_g_co=alpha_g_co+est_co; \%estımatıon of having acceptable cooperative channel

for $\mathrm{f}=1: \mathrm{M}$

end

$\mathrm{N}_{\mathrm{s}} \mathrm{s}(: \mathrm{f})=\mathrm{N}$ _selection; \%Rearrange the matrix $N$ selectıon in 3 dimentıons

beta_g_co $=$ beta_g_co $+N_{\text {N_s }}$-est_co;

alpha_co=alpha_co+sent; \% Number of sent and recerved packets are updated beta_co=beta_co+recv-sent;

p_sel=select./(counter); \% update Probability of being selected

alpha_se_co=alpha_se_co+select; \% Number of time a relay has been selected in $10 \overline{0}$ frame

beta_se_co=beta_se_co+counter-select;

end \%frame ends

\%iteration ends

end

\%calculating the average 
tco_avg=tco_avg./T; \% the reult is a three dimentıonal matrix whose dimentions are user, relay and iteration( $(\mathrm{\jmath})$

save tco_avg; \%Saves the result

end

end

\% This function provides us with the plot of average aggregated queue lengths of for five

$\%$ policies as follows. The main calculations are performed in function $f()$

$\%$ which is invoked in this function.

\%TR: Secure Throughput Optımal

\%TO: Non-Secure Throughput Optrmal

\%ıTO: Secure Instantaneous Throughput Optımal

\%iso: Throughput Optımal with Pure Trusted Relays

$\%$ NC: Non-Cooperative

$\%$ Results are averaged for Repl replucations.

function main

$\mathrm{N}=12 ; \%$ number of users

$\mathrm{K}=8 ; \%$ number of relays

repl $=30$; \%run replacations

$\% \operatorname{Tr}=[.2 *$ ones $(1,12) ; .2 *$ ones $(1,12) ; .2 *$ ones $(1,12) ; .2 *$ ones $(1,12) ; .2 *$ ones $(1,12) ; .2 *$ ones $(1,12) ; .2 *$ ones $(1,12) ; .2 *$ ones $(1,12)]$;

$\operatorname{Tr}=[$ ones $(1,12) ;$ ones $(1,12) ;$ ones $(1,12) ;$ ones $(1,12) ; .8 *$ ones $(1,12) ; .6 *$ ones $(1,12) ; .4 *$ ones $(1,12)$ $; .2 *$ ones $(1,12)]$;

for $\mathrm{j}=1: 40 \%$ Calculations are for 40 different arrvvals

$$
\begin{aligned}
& \operatorname{TO}(\mathrm{j})=0 ; \\
& \operatorname{iTO}(\mathrm{j})=0 ; \\
& \operatorname{NC}(\mathrm{j})=0 ; \\
& \operatorname{TR}(\mathrm{j})=0 ; \\
& \operatorname{iso}(\mathrm{j})=0 ;
\end{aligned}
$$

end

for $j=1$ :repl

for $\mathrm{i}=1: 40$

\%lambda $f$

$\mathrm{t}(\mathrm{i})=5 * 1.09^{\wedge} \mathrm{i}$;

$[\mathrm{a}, \mathrm{b}, \mathrm{c}, \mathrm{d}, \mathrm{e}]=\mathrm{f}(\mathrm{N}, \mathrm{K}, \mathrm{t}(\mathrm{i}), \mathrm{Tr}) ; \%$ Average aggregated queue length for arrvval $\mathrm{t}(\mathrm{\imath})$, and trust $T r$

$\mathrm{TO}(\mathrm{i})=\mathrm{a}+\mathrm{TO}(\mathrm{i})$;

$\mathrm{iTO}(\mathrm{i})=\mathrm{b}+\mathrm{iTO}(\mathrm{i}) ; \%$ Trust may be the out put of any trust establishment mthod between $[0,1]$

$\mathrm{NC}(\mathrm{i})=\mathrm{c}+\mathrm{NC}(\mathrm{i})$;

$\mathrm{TR}(\mathrm{i})=\mathrm{d}+\mathrm{TR}(\mathrm{i})$;

iso(i) $=\mathrm{e}+$ iso(i);

end

end

figure;

hold;

$\operatorname{plot}\left(\mathrm{t}, \mathrm{TO} / \mathrm{repl},{ }^{*}-^{\prime}\right)$; 


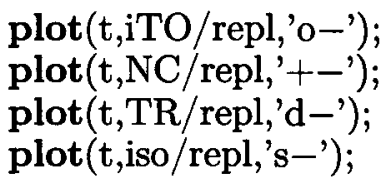

$\%$ figure;

$\%$ hold;

$\%$ plot(t,length_on_off,'*-');

$\%$ plot (t,length $\overline{2}_{-}$on_off, 'o-');

end

$\%$ This function provides us with the average aggregated queue lengths of for five

$\%$ policies as follows. The main calculations are performed in function $f()$

$\%$ which is invoked in this function.

\%TR: Secure Throughput Optimal

\%TO: Non-Secure Throughput Optimal

\%थTO: Secure Instantaneous Throughput Optımal

\%iso: Throughput Optrmal with Pure Trusted Relays

$\%$ NC: Non-Cooperative

\% It takes trust Tr, arrival rate $p$, Number of users $N$ and number of relays $K$ and gives

the average aggregated backlogs for five polvcies as mentioned

function $[\mathrm{TO}, \mathrm{iTO}, \mathrm{NC}, \mathrm{TR}, \mathrm{iso}]=\mathrm{f}(\mathrm{N}, \mathrm{K}, \mathrm{p}, \mathrm{Tr})$

$\mathrm{SNR}=1$

Q_TO=zeros $(\mathrm{N}, 1)$;

$\mathrm{Q}_{-} \mathrm{iTO}=\operatorname{zeros}(\mathrm{N}, 1) ; \%$ queue backlog for five polıcıes as defined

$\mathrm{Q}_{-} \mathrm{NC}=\operatorname{zeros}(\mathrm{N}, 1)$;

$\mathrm{Q}_{-}^{-} \mathrm{TR}=\operatorname{zeros}(\mathrm{N}, 1)$;

$\mathrm{Q}_{\text {_iso }}=\operatorname{zeros}(\mathrm{N}, 1)$;

time $=5000$;

$\mathrm{x}_{-} \mathrm{rel}(1,:)=[2,0] ; \%$ location of relays which is fixed

$\mathrm{x}_{-}$rel $(2,:)=[2,2] ; \%$ during the simulation

$x_{-}$rel $(3,:)=[0,2]$;

$x_{-}$rel $(4,:)=[-2,2]$;

x_rel $(5,:)=[-2,0]$;

$x$ rel $(6,:)=[-2,-2]$;

$x_{-}$rel $(7,:)=[0,-2]$;

$x_{-}$rel $(8,:)=[2,-2]$;

$\mathrm{x}=\operatorname{randint}(\mathrm{N}, 2,[-55])$;

$\% x(9,:)=\operatorname{randsrc}(1,2,[-55])$;

$\% x(10,:)=\operatorname{randsrc}(1,2,[-55])$;

$\% x(11,:)=\operatorname{randsrc}(1,2,[-55])$;

$\% x(12,:)=\operatorname{randsrc}(1,2,[-55])$;

for $\mathrm{i}=1: \mathrm{N}$

if $(x(i, 1)==0 \quad \& \& x(i, 2)==0)$

$$
\mathbf{x}(i, 1)=\mathbf{x}(\mathbf{i}, 1)+1 \text {; }
$$

end

end 


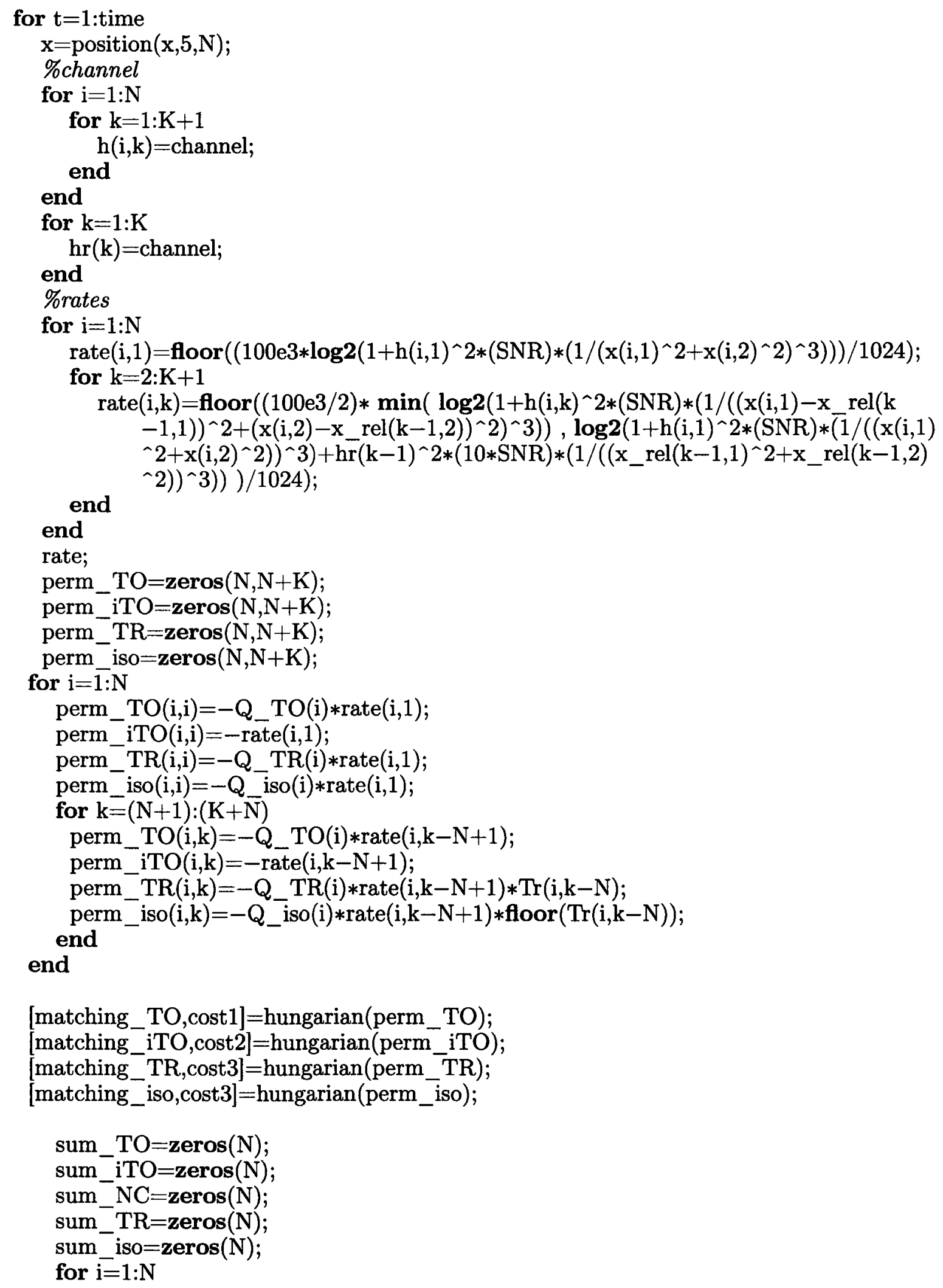




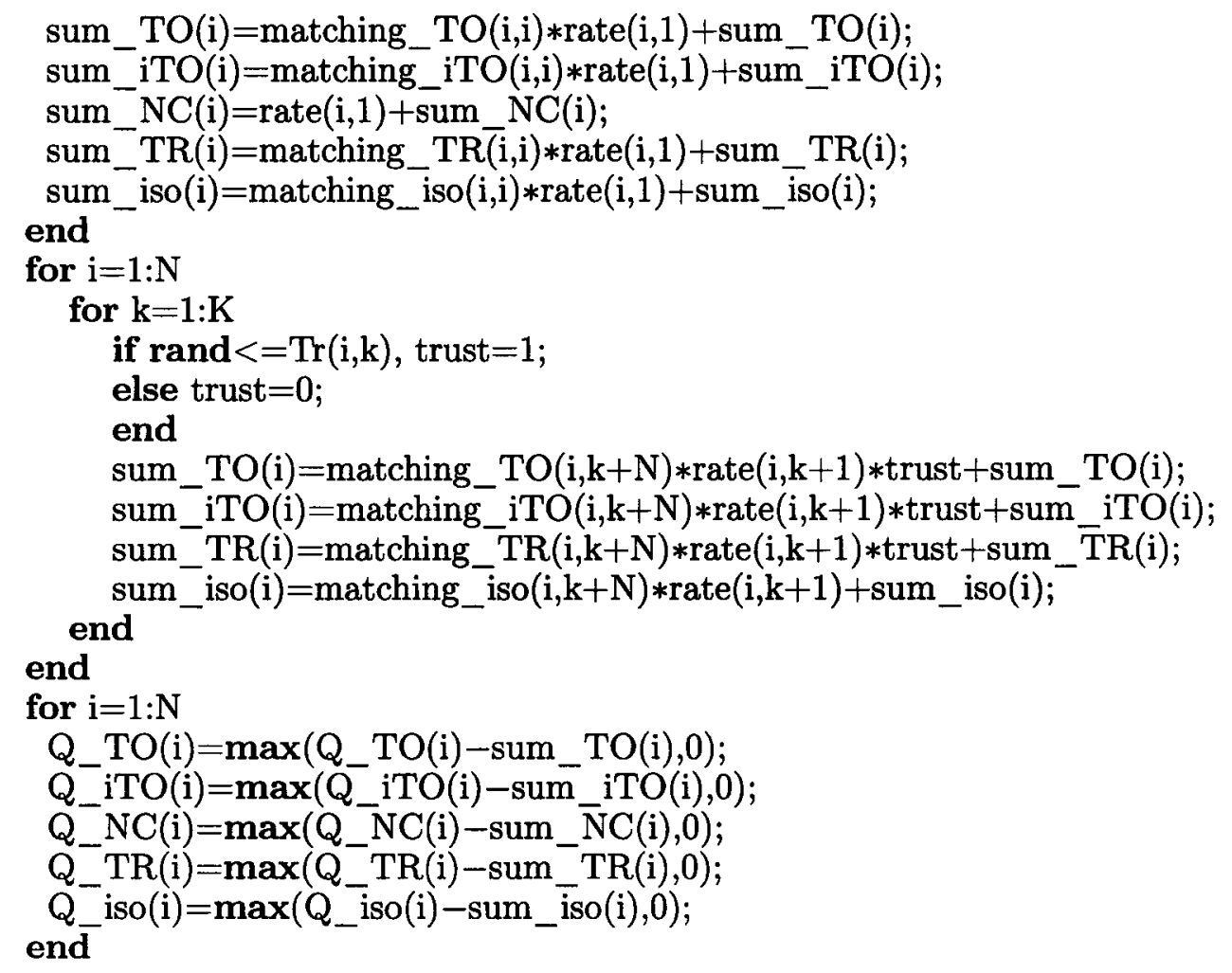

for $\mathrm{i}=1: \mathrm{N} \%$ calculates arrvvals for $N$ users

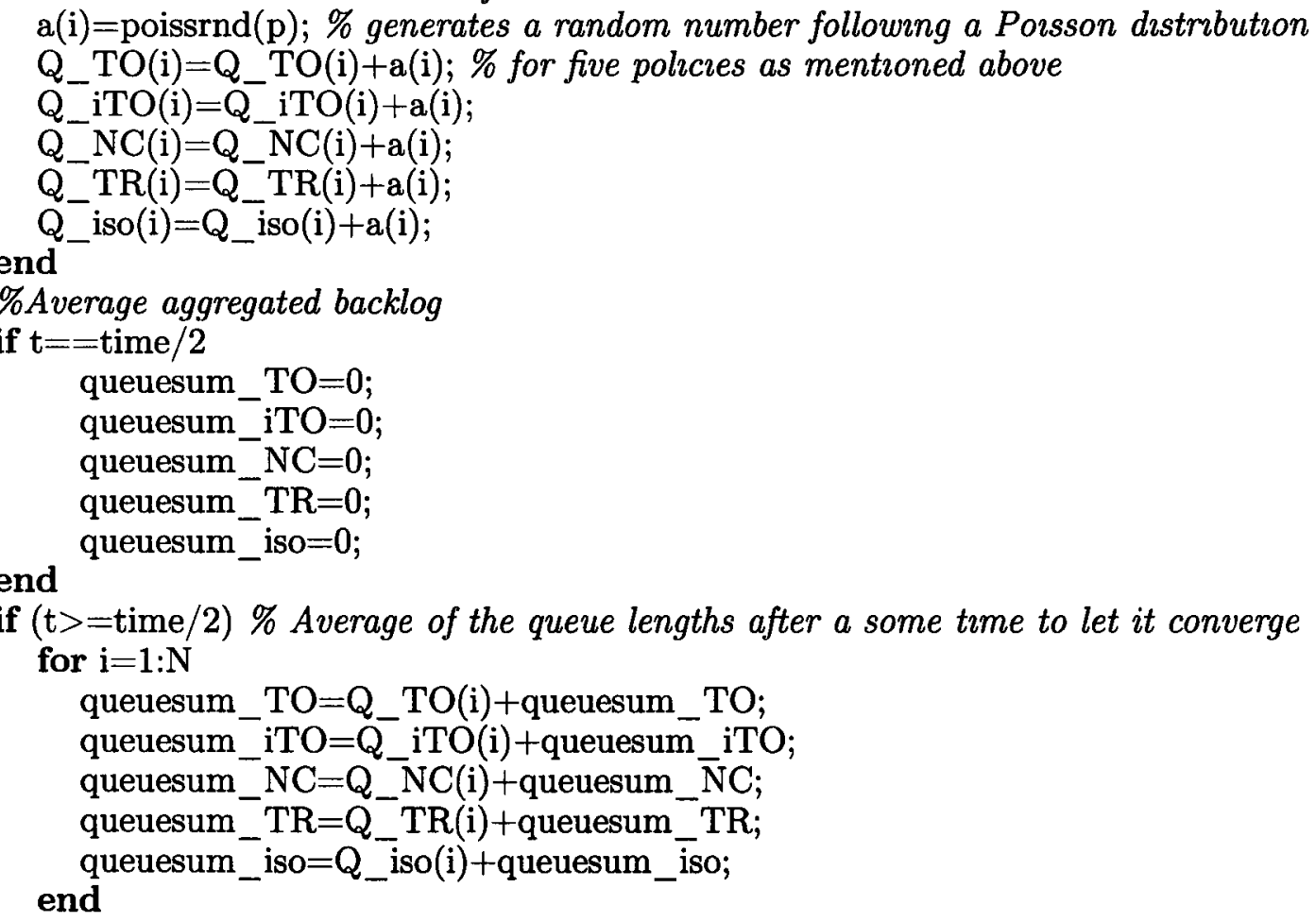


end

end

$\mathrm{TO}=$ queuesum_TO $/(\mathrm{N} *($ time $/ 2))$;

$\mathrm{iTO}=$ queuesum_iTO $/(\mathrm{N} *($ time $/ 2))$;

$\mathrm{NC}=$ queuesum_ $\mathrm{NC} /(\mathrm{N} *($ time $/ 2))$;

$\mathrm{TR}=$ queuesum_TR/(N*(time $/ 2))$;

iso=queuesum_iso $/(\mathrm{N} *($ time $/ 2))$;

\%This Function gets the current two dimentional position $x$, Size of the grid 2M, and number of time slots $N$. The out put is a vector of

\%position with length $N$, forming a path on a Size of the Grid is $2 M+1 * 2 M+1$

\%we consider equal probability movement upward, downward, to left and right. The resultıng posit

function $y=\operatorname{position}(x, M, N)$

$\mathrm{y}=\mathrm{x}$;

for $\mathrm{i}=1: \mathrm{N}$,

$\mathrm{r}=\operatorname{rand}(1) ; \%$ Generates a random number in $[0,1]$

if $(\mathrm{x}(\mathrm{i}, 1)==-\mathrm{M} \& \& \mathrm{x}(\mathrm{i}, 2)==-\mathrm{M}) \%$ If on the buttom leftcorner, go right and left equall prob. $=0.5$

if $(\mathrm{r}<=1 / 2)$

end

$\mathrm{y}(\mathrm{i}, 1)=\mathrm{x}(\mathrm{i}, 1)+1 ; \% g \circ$ reght

if $(r>1 / 2)$

end

$\mathrm{y}(\mathrm{i}, 2)=\mathrm{x}(\mathrm{i}, 2)+1 ; \%$ go up

elseif $(x(i, 1)==-M \& \& x(i, 2)==M)$

if $(r<=1 / 2)$

$\mathrm{y}(\mathrm{i}, 1)=\mathrm{x}(\mathrm{i}, 1)+1 ; \%$ If on the top left corner, go right with prob. $=0.5$

end

if $(r>1 / 2)$

$\mathrm{y}(\mathrm{i}, 2)=\mathrm{x}(\mathrm{i}, 2)-1 ; \%$ If on the top left corner, go down with prob. $=0.5$ end

elseif $(\mathrm{x}(\mathrm{i}, 1)==\mathrm{M} \& \& \mathrm{x}(\mathrm{i}, 2)==\mathrm{M}) \%$ If on the top right corner, go left with prob .$=0.5$

if $(\mathrm{r}<=1 / 2)$

$\mathrm{y}(\mathrm{i}, 1)=\mathrm{x}(\mathrm{i}, 1)-1 ; \%$ If on the top right corner, go down with prob. $=0.5$

end

if $(\mathrm{r}>1 / 2) \%$ go down

$y(i, 2)=x(i, 2)-1$;

end

elseif $(x(i, 1)==M \& \& x(i, 2)==-M) \%$ If on the buttom right corner,

end

if $(\mathrm{r}<=1 / 2)$

end

$\mathrm{y}(\mathrm{i}, 1)=\mathrm{x}(\mathrm{i}, 1)-1 ; \%$ go left $\operatorname{Prob}=0.5$

if $(r>1 / 2)$

end

$\mathrm{y}(\mathrm{i}, 2)=\mathrm{x}(\mathrm{i}, 2)+1 ; \%$ go right Prob. $=0.5$

end 


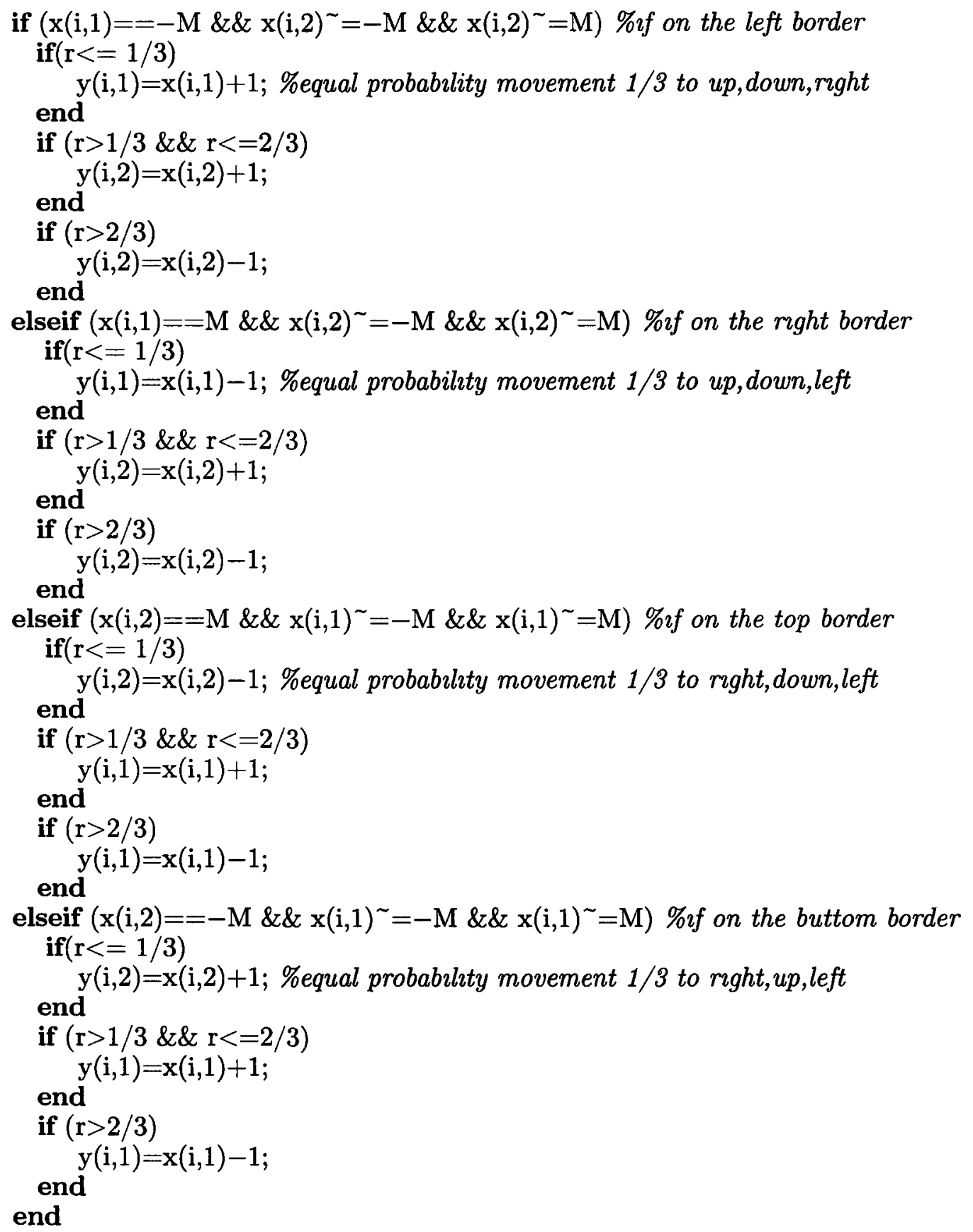

if $(\mathrm{x}(\mathrm{i}, 1)==1 \& \& \mathrm{x}(\mathrm{i}, 2)==0) \%$ Preventing from going to the orign otherwise, if $(r<=1 / 3)$

$\mathrm{y}(\mathrm{i}, 1)=\mathrm{x}(\mathrm{i}, 1)+1 ; \%$ we have zero distance and infinite $S N R$ in the simulation 


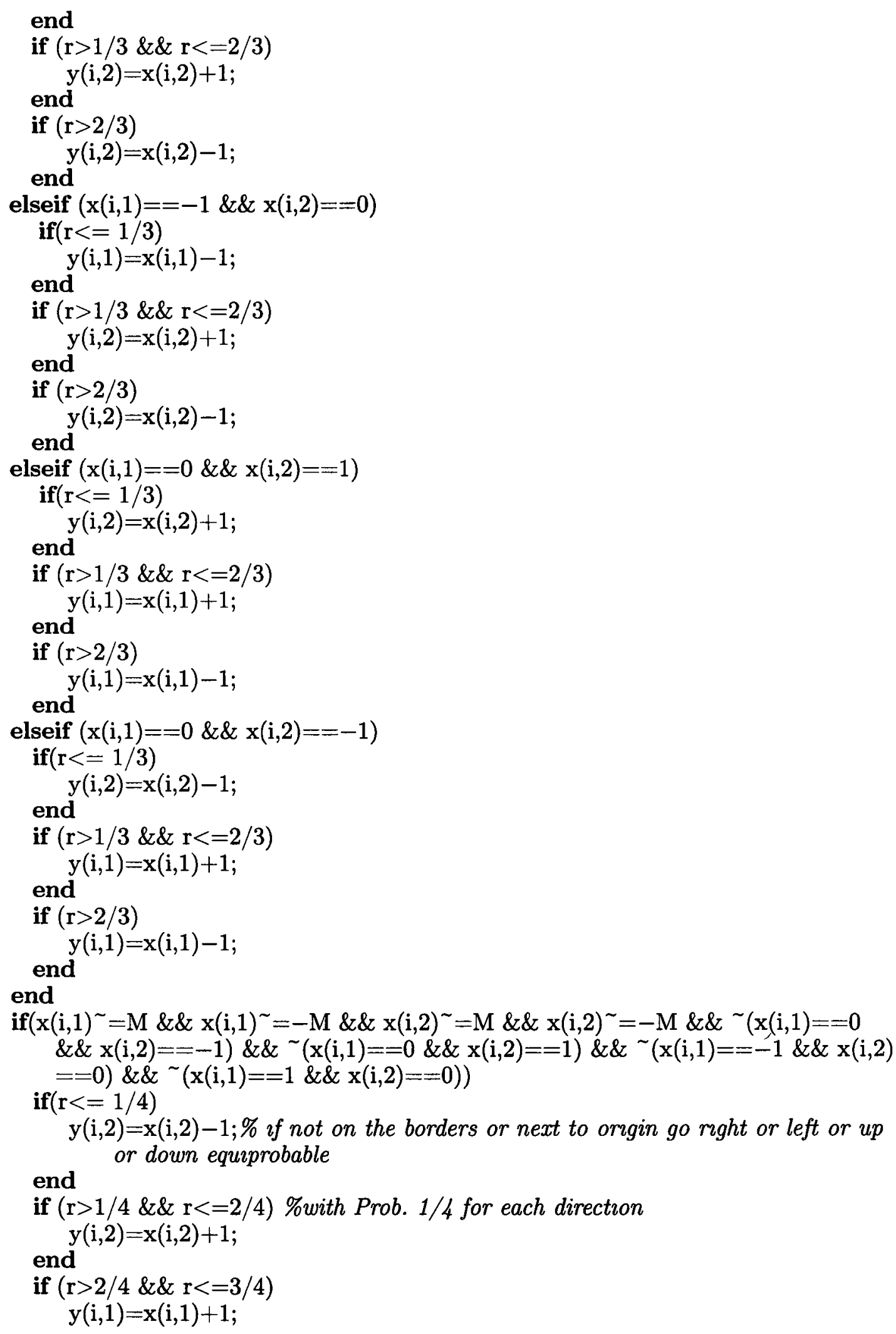




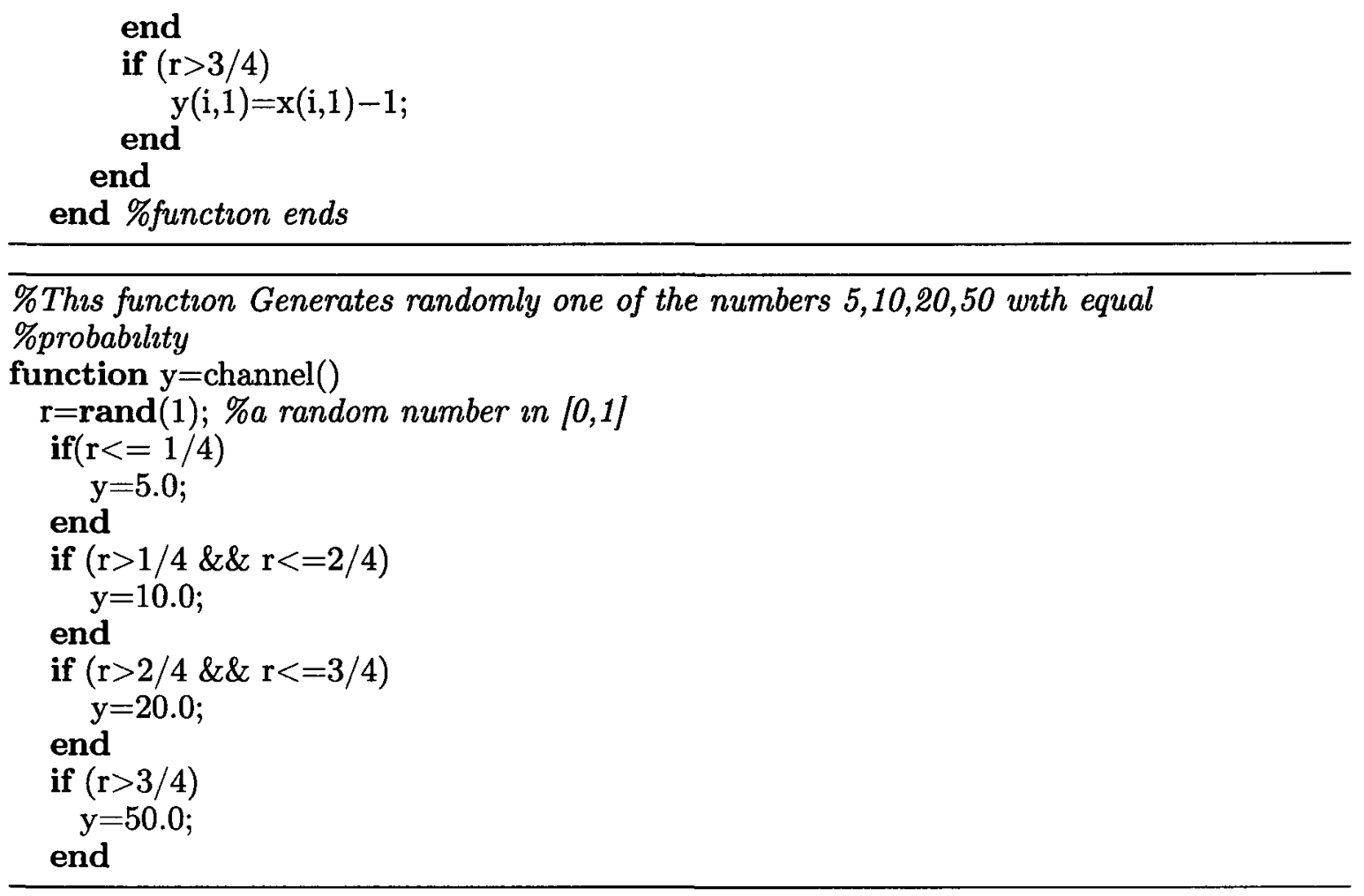

